\title{
Fluid Physics and Surrogate Modeling of a Low Reynolds Number Flapping Rigid Flat Plate
}

\author{
Pat Trizila $^{1 *}$, Chang-Kwon Kang ${ }^{1 \dagger}$, Hikaru Aono ${ }^{1 \star}$, Miguel Visbal ${ }^{2 \S}$, and Wei Shyy ${ }^{1 * *}$ \\ ${ }^{1}$ Department of Aerospace Engineering, University of Michigan, Ann Arbor, Michigan 48109 \\ ${ }^{2}$ U.S. Air Force Research Laboratory, Wright-Patterson Air Force Base, Ohio 45433
}

Two- and three-dimensional, low aspect ratio ( $\mathrm{AR}=4)$, hovering airfoil/wing aerodynamics at low Reynolds number $(\operatorname{Re}=100)$ are numerically investigated. Regarding fluid physics, in addition to the well-known leading edge vortex and wake-capture mechanisms, a persistent jet, induced by the shed vortices in the wake during previous strokes, and tip vortices can significantly influence the lift and power performance. While in classical stationary wing theory the tip vortices are seen as wasted energy, here, they can interact with the leading edge vortex to contribute to the lift generated without increasing the power requirements. Using surrogate modeling techniques, the two- and three-dimensional time averaged aerodynamic forces were predicted well over a large range of kinematic motions when compared to the Navier-Stokes solutions. The combined effects of tip vortices, leading edge vortex, and jet can be manipulated by the choice of kinematics to make a three-dimensional wing aerodynamically better or worse than an infinitely long wing. The environmental sensitivity during hovering for select kinematics is also examined. Different freestream strengths and orientations are imposed with the impact on vortex generation and wake interaction investigated with implications on the applicability of quasi-steady models.

\section{Introduction}

Micro air vehicles (MAVs) with a maximal dimension of $15 \mathrm{~cm}$ or less and a flight speed of $10 \mathrm{~m} / \mathrm{s}$ are interests of both military and civilian applications. Equipped with a video camera or a sensor, these vehicles can perform surveillance and reconnaissance, and environmental and biochemical sensing at remote or otherwise hazardous locations. In addition, from the scaling laws ${ }^{1}$, a MAV's payload is very limited; sensors, batteries, communications equipment, and means of sustained propulsion will have to compete for precious cargo space only in as much as that they will directly help defined mission statements. Research regarding MAVs is growing as there are still many open challenges in theory and in practice.

There are three different design approaches in MAV development. (i) The fixed wing vehicles $^{2}$ resemble miniaturized versions of conventional fixed wing aircraft except that flexible wings are often utilized. (ii) Small rotor based ${ }^{3}$ vehicles resembling helicopters are also pursued, where often multiple rotors are used. One defining characteristic for which these vehicles are valued is their ability to hover, allowing for monitoring systems which can remain stationary or

\footnotetext{
${ }^{*}$ Graduate Research Assistant, Member AIAA

${ }^{\dagger}$ Graduate Research Assistant, Member AIAA

* Post-doctoral Scholar, Member AIAA

$\S$ Technical Area Leader, Computational Sciences Branch, AFRL/RBAC, Associate Fellow AIAA.

** Clarence L. "Kelly" Johnson Collegiate Professor and Chair, Department of Aerospace Engineering, Fellow AIAA.
} 
follow targets as the situation dictates. (iii) The flapping wing variety of MAVs ${ }^{1,4}$, of interest in the current study, take a cue from nature and attempt to mimic the success achieved by insects, birds, and bats ${ }^{5-7}$. Common to all three classifications is the sensitivity to perturbations in the environment such as wind gusts, assessing the impact of transition to turbulence which due to the scales involved may or may not be relevant and can be difficult to gauge a priori, the need for coupled fluid-structure interaction capabilities to capture the changes in the vehicle induced by the flow, the advanced control schemes needed to successfully fly these vehicles autonomously, and the understanding of the unsteady flow mechanisms present and their respective roles in the aerodynamic loadings on the MAV.

The study of flapping wing flyers with all of their intricacies is challenging. However, as summarized in references 1,4 , and 8-16, significant progress has been made in both engineering and biological communities. Computational studies of realistic wing body configurations of a hornet $^{17}$, hawkmoth ${ }^{18}$, fly and thrip ${ }^{1,19-21}$ have been investigated from various angles. On hovering flight, Liu and Sun ${ }^{22}$ have measured the time course of body and wing kinematics of droneflies using three-dimensional high speed video and have input these kinematics as boundary conditions in Navier-Stokes simulations. They found out that droneflies use mainly the delayed stall mechanism to generate weight-supporting lift, unlike fruit flies that use both drag and lift to stay aloft. Wang et al. ${ }^{19}$ compared two-dimensional computational simulations of an ellipse against experimental results. Tang et al. ${ }^{23}$ investigated the effects of Reynolds number, reduced frequency, and flapping kinematics on the flow structure and aerodynamics. PIV measurements were carried out by Kurtulus et $a l^{24}$, detailing experimental observations of flapping wing flight with hovering kinematics. Two-dimensional computational studies were examined by Bos et al. ${ }^{25}$ of different hovering kinematics found in the literature while Trizila et $a l .{ }^{26}$ gauged the sensitivity of the kinematic parameters by tying the consequences to the resulting unsteady mechanisms and finally to the aerodynamic loadings. Moreover, Sane and Dickinson ${ }^{27}$ carried out a comprehensive experimental study of five kinematic hovering variables and compared their results to the quasi-steady predictions. While the mean lift value over the course of a wing cycle matched reasonably, the instantaneous values differed significantly. The implication here is that a potential control device, for which moments and therefore instantaneous forces are important, would not be able to use quasi-steady approximations as a feasible reduced order model to be used in real time calculations. They also showed that the mean drag was greatly underestimated which would in turn under predict power required estimates. Zbikowski ${ }^{28}$ investigated the theoretical aerodynamic modeling of hovering micro air vehicles proposing a framework consisting of Karman-Sears unsteady wing theory and an approach via velocity potentials. Hsieh et al. ${ }^{29}$ look at advanced, delayed, and symmetric rotation and break down the lift coefficients in terms of the lift caused by vorticity, wing velocity, and wing acceleration. They find that while the symmetric rotation has the most vorticity lift, the optimal lift is found with advanced rotation. Taira and Colonius ${ }^{30}$ studied low aspect ratio, impulsively started flat plates at Reynolds numbers from 300 to 500 and, relevant to the current study, saw that when the aspect ratio was four, the mid-span flow behavior in two dimensions was vastly different from the three-dimensional cases. That same study also saw a performance decrease induced by the tip vortices which in flapping wing flight can in fact provide a positive contribution, as will be elaborated on in the present investigation.

As summarized by Shyy et al. ${ }^{1}$, several lift enhancement mechanisms have been identified including leading edge vortex, pitch-up rotation, wake-capture and clap-and-fling. Delayed stall comes about as a leading edge vortex ${ }^{31}$ (LEV)forms on the upper surface of the airfoil and 
attaches even when the wing achieves a high angle of attack. This in turn produces a low pressure region on the upper surface which increases the lift. Wake-capture refers to when the airfoil switches direction and encounters the previously shed wake, which can then be taken advantage of, if at the proper orientation. An important unsteady mechanism, though not performance enhancing, is that of a vortex induced jet. This flow feature was seen experimentally by Freymuth ${ }^{32}$ but has not been adequately studied.

A model problem has been chosen to probe the unsteady flight mechanisms and their relationship with the hovering kinematics, and the implications on three-dimensional aerodynamics. It is generally held that as the aspect ratio is reduced, a potentially strong spanwise variation in the fluid field results. The issue of wing rotation about a joint, due to the centripetal accelerations and extra degrees of freedom, are not investigated. Despite their simplified nature, the chosen kinematics are directly relevant to future MAVs at this scale, and the impact of the flow physics can be extrapolated to other real or imagined cases.

In this work, we assess both two- and three-dimensional flapping wing fluid physics at Reynolds number $(\mathrm{Re})$ of 100 highlighting the role of the various fluid physics, including leading edge vortex, wake-capture, tip vortices and induced jet (see figure 1), and their overall aerodynamic implications on lift and power during hovering. The Reynolds number in the current study is relevant to small insects and future MAVs at this scale. Prior studies in the literature applicable to hovering in this flow regime include those of Freymuth ${ }^{32}$, Fry et al. ${ }^{33}$, Sane and Dickinson ${ }^{27}$, Sun and Tang ${ }^{34}$, Tiara and Colonius ${ }^{30}$, Wang et al. ${ }^{19}$, and Shyy and Liu ${ }^{20}$. As will be shown, despite increased interest in the area of flapping wing flight, there are a number of aspects left unaddressed. Regarding the generalization of ideas presented to higher Reynolds numbers, it has previously been noted ${ }^{1,20}$ that, as the sizing and scaling parameters vary, the flow structures can qualitatively differ between those found for small flyers versus larger flyers.

The main efforts of the study show: i) Tip vortices in low Reynolds number flapping wing flight, depending on the flapping kinematics, may have a significant impact on the aerodynamic loading. As presented by Shyy et al. ${ }^{35}$ in the form of a letter, with details expanded in the current paper, these tip vortices, counter to classical steady-state theory for finite wings, can positively contribute to the performance of the wing instead of being considered wasted energy. The tip vortices affect the impact of the leading edge vortex via modified angle-of-attack and the anchoring of the coherent vortex structure to the wing; they also influence the strength of the induced jet, to be described next. ii) The presence of an induced jet, observed in the experimental observations of Freymuth $^{32}$, in two-dimensions is found to decrease lift during the wing-wake interactions. iii) The role of kinematics in two-dimensional and three-dimensional geometry is explored. To establish a systematic framework to probe the interplay between flapping kinematics and aerodynamics (time-averaged lift and power), the surrogate modeling technique is employed to represent their relationship. As will be presented, the surrogate models offer a global perspective between aerodynamics and flapping kinematics, including an assessment of the relative importance of these kinematic parameters. In this fashion, trends can be more clearly observed. For example, the surrogate model reveals that for a considerable portion of the design space lift is accentuated while the drag, and subsequently power required, is largely unaffected. The characteristics of two-dimensional versus three-dimensional hovering can also be probed with desirable clarity. Utilizing the surrogate model, we can clearly identify regions where twoor three-dimensional wing perform better aerodynamically, or where the effect of the aspect ratio is significant. The tool offers new insight into the performance characteristics and helps seek 
physical mechanisms responsible for the observed behavior. The surrogate process also provides an advantage over isolated case studies in building a more complete understanding of the context of the conclusions, and therefore the relevance/limitations of comparisons with other studies' conclusions can be better established. In this paper, only translational flapping is considered; the rotational flapping will be investigated in the future.
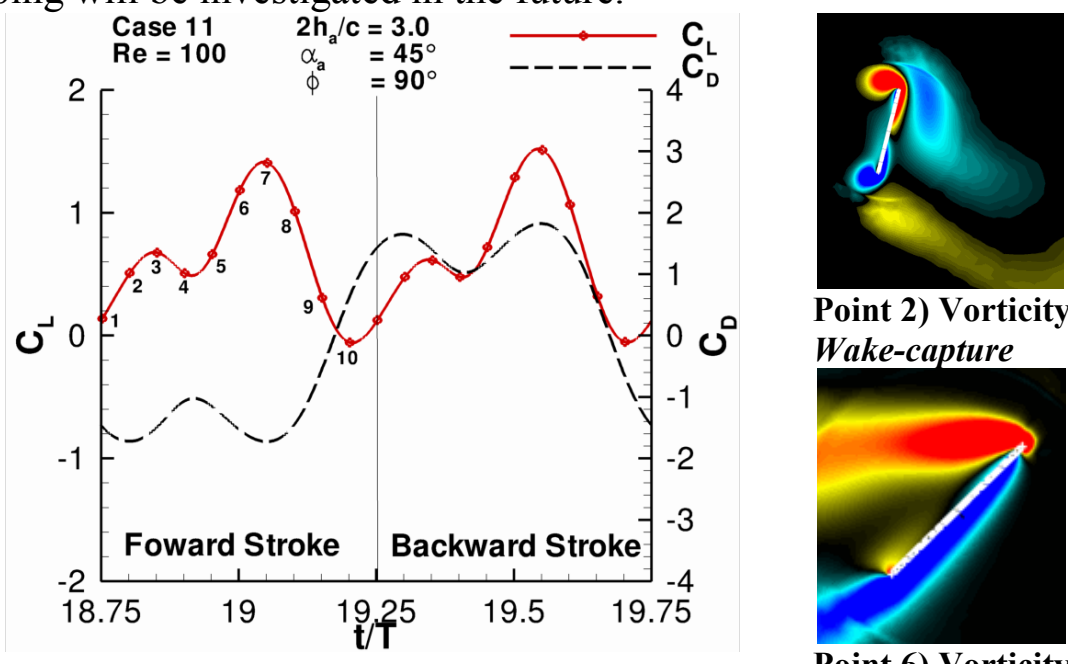

Point 2) Vorticity Contours

Wake-capture

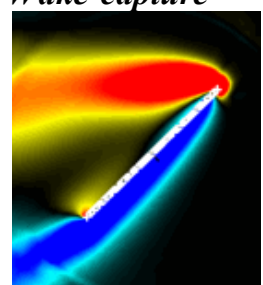

Point 6) Vorticity Contours

Delayed Stall

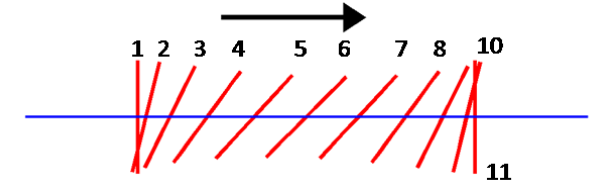

$\begin{array}{lllllllllll}-2.5 & -2 & -1.5 & -1 & -0.5 & 0 & 0.5 & 1 & 1.5 & 2 & 2.5\end{array}$

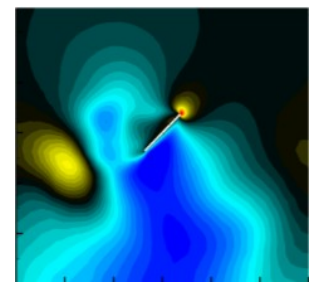

Point 6) Vertical Velocity

Jet Interaction

FIGURE 1. Illustration of the lift and drag coefficients for a two-dimensional flat plate synchronized hovering case with $2 h_{a} / c=3.0, \alpha_{a}=45^{\circ}$, and $\phi=90^{\circ}$ and the corresponding airfoil positions. Three flow field shots illustrating the unsteady aerodynamics are emphasized.

In the remaining paper, the methodology followed in this study will be first presented. Due to surrogate modeling not being extensively used in fluid dynamic studies, brief introductions to the nomenclature and overview of the methods are introduced while more detailed discussion is left for the references. It is emphasized that the surrogate models' main role in the study was as a tool to help in the analysis of the physics, and while optimization is a straightforward extension of the current work, it was not a primary focus. After the methodology the results are divided into sections relevant to lift, power, and trade-offs between the two objectives. Together with force histories and flow fields at time instants of interest, comprehensive information of hovering aerodynamics at $R e=100$ are obtained. Four regions in the design space are detailed more thoroughly based on the large discrepancies found between the 2D and 3D lift. The flow physics are explored with special attention to how the tip vortices impact the flow. After this, power is studied again taking a global perspective. Sensitivities of the lift and power objectives to the kinematic parameters is quantified and the competition of effects illustrated. Varied freestream strengths are introduced to gauge environmental sensitivity, and lastly conclusions are summarized and appendices offering supplemental materials offering more detailed information of the various aspects are documented. 


\section{Methodology}

\subsection{Computational fluid dynamic modeling}

The governing equations are the unsteady Navier-Stokes equations with constant density and viscosity; the incompressible versions are shown in Eqs. (1) and (2) written in indicial form.

$$
\begin{gathered}
\frac{\partial}{\partial x_{j}}\left(u_{j}\right)=0 \\
\frac{\partial}{\partial t}\left(u_{i}\right)+\frac{\partial}{\partial x_{j}}\left(u_{j} u_{i}\right)=-\frac{1}{\rho} \frac{\partial p}{\partial x_{i}}+v \frac{\partial}{\partial \mathrm{x}_{j}}\left(\frac{\partial u_{i}}{\partial x_{j}}\right)
\end{gathered}
$$

Here $u_{i}$ is the velocity vector, $x_{i}$ is the Cartesian position vector, $t$ is time, $\rho$ is density of the fluid, $p$ is pressure, and $v$ is the kinematic viscosity of the fluid. Two codes with vastly different methodologies are used in this study, Loci-STREAM and FDL3DI and are discussed below.

Loci-STREAM ${ }^{36}$, used to produce the CFD solutions, is a three-dimensional, unstructured, pressure-based finite volume solver. It employs implicit first or second order time stepping, treats convection terms using the second order upwind-type scheme ${ }^{37,38}$, pressure and viscous terms using second order schemes. The system of equations resulting from the linearized momentum equations are fast to converge ${ }^{38}$ and are handled with the symmetric Gauss-Seidel ${ }^{39}$ solver which has relatively low memory requirements. The pressure equation ${ }^{36}$ is slower to converge, and is handled by the PETSc Krylov ${ }^{39}$ solvers with Jacobi preconditioning. The Loci framework is by design highly parallelizable and can take advantage of multiple processors, see Luke and George ${ }^{40}$ for a more detailed discussion on rule-based software.

FDL3DI is a higher order finite difference solver. It uses a density based formulation, and handles numerical instabilities associated with the higher $\left(6^{\text {th }}\right.$ or $\left.8^{\text {th }}\right)$ order scheme with filtering techniques. For further discussion the reader is referred to Visbal and Gaitonde ${ }^{41,42}$. These two CFD codes are cross-validated in while grid and temporal sensitivities were explored in Trizila et $a l^{26}$. Based on these critical examinations, we have confidence in the fidelity of the methods employed.

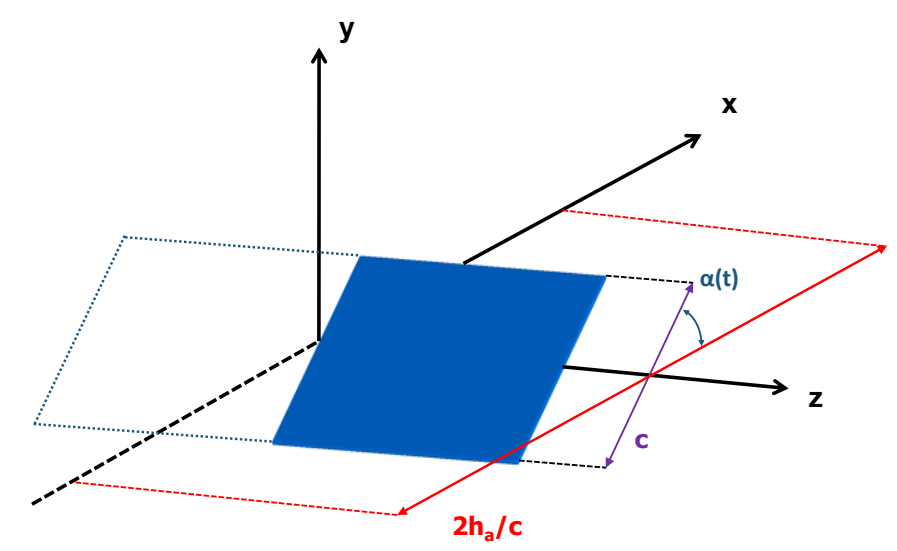

FIGURE 2. Illustration of the kinematic parameters for normal hovering.

The translational and rotational airfoil/grid motions are defined as Eqs. (3) and (4), and shown in figure 2 .

$$
\begin{gathered}
h(t)=h_{a} \sin (2 \pi f t) \\
\alpha(t)=\alpha_{0}-\alpha_{a} \sin (2 \pi f t+\phi)
\end{gathered}
$$


Here $h(t)$ and $h_{a}$ are the dimensional translational position and plunging amplitude, respectively. The angular orientation, time-averaged angle, and angular amplitude are $\alpha(t), \alpha_{0}$, and $\alpha_{a}$, respectively. The pitching is about the center of the rigid airfoil. The phase lag between the two motions is $\phi$, and the motion frequency is denoted $f$ whereas the time is again $t$. The three kinematic parameters (called "design variables" in surrogate modeling), $h_{a}, \alpha_{a}$, and $\phi$ can be varied independently.

Due to the kinematic constraints there are only two relevant non-dimensional groups in the incompressible case. The plunging amplitude to chord ratio, $2 h_{a} / c$, and the Reynolds number:

$$
R e=\frac{U_{\mathrm{ref}} L_{\mathrm{ref}}}{v}=\frac{\left(2 \pi f h_{a}\right) c}{v}
$$

The reference velocity, $U_{\text {ref }}$, in this case is the maximum translational velocity, defined by the flapping frequency, $f$, and the plunging amplitude, $h_{a}$. Since $R e$ is being held constant, $h_{a}$ and $f$ are not independent. Note that the reduced frequency, $k$, is not emphasized here as, in the absence of a free stream, it contains the same information as the plunging amplitude ratio whereas if $U_{\text {ref }}$ is instead set equal to zero the reduced frequency is always infinite.

$$
k_{\text {hovering }}=\frac{\omega L_{\mathrm{ref}}}{2 U_{\mathrm{ref}}}=\frac{2 \pi f c}{2\left(2 \pi f h_{a}\right)}=\frac{c}{2 h_{a}}
$$

Figure 3 shows the grid distribution near the flat plate, and the applied boundary conditions over the computational domain. The thickness of the flat plate is $0.02 c$, and the flat plate is rectangular, i.e. there is no variation in spanwise direction $(z$-axis). For the finite wing simulations $\mathrm{AR}=4$. The half-span length from the symmetry plane to the wingtip is $2 c$, the leading, and trailing edges are rounded using a half circular shape, while the wingtip is flat. The fine details of the shape are not seen to have a large influence on the resulting aerodynamics. A more pronounced shape effect is a comparison between a flat plate and a $15 \%$ thickness ellipse, Trizila et $a l .{ }^{43}$ and Shyy et $a l .{ }^{1}$, which saw differences in the magnitude of the force generation but the same qualitative force and flow behavior. The outer boundary is located at $25 c$ away from the flat plate, and the outer boundary plane opposite to the symmetry plane at $15 c$. At all non-symmetry plane outer boundaries, inlet conditions with zero velocity conditions are imposed.
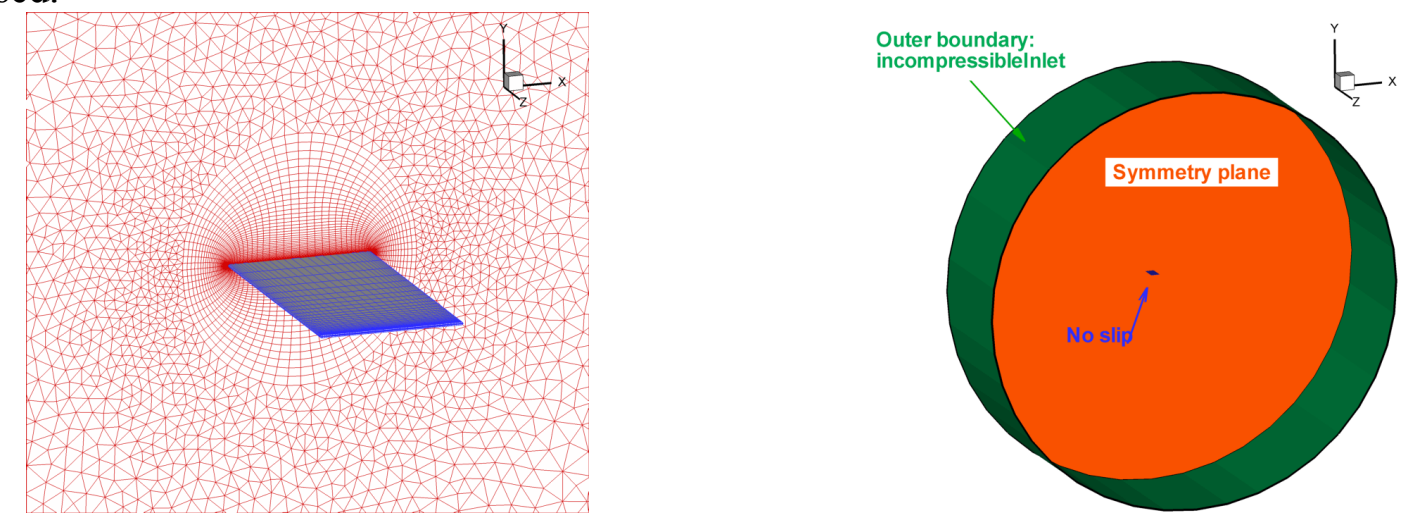

FIGURE 3. (Left): Grid distribution on the flat plate, and on the symmetry plane. (Right): Boundary conditions assigned on the computational domain. The outer boundary plane opposite to the symmetry plane has not been shown due to visibility also has the incompressibleInlet boundary condition.

\subsection{Surrogate modeling}

The motivation behind surrogate models is to replace costly objective function evaluations, a quantity of interest such as time averaged lift obtained via detailed CFD solutions or substantial 
experimental setups, with inexpensive approximations of sufficient fidelity. Overview of surrogate modeling techniques and selected applications can be found in references 44-46. The process starts with choosing number and locations of training points in the "design space" (here meaning the three-dimensional space encompassing the three kinematic parameters); this is known as constructing the design of experiment (DOE). Once the training points are chosen, the objective functions must be evaluated; depending on the context this can be done computationally, analytically, and/or experimentally. After the objective functions have been obtained for the selected training points, the surrogate models can be built. Popular models are polynomial response surfaces $(\mathrm{PRS})^{47}$, Kriging $^{48}$, radial basis neural networks $(\mathrm{RBNN})^{49}$, support vector regression $(\mathrm{SVR})^{50}$ models and various combinations thereof. After the models are constructed, appropriate error measures can be adopted to construct a weighted average surrogate (WAS) ${ }^{51,52}$. Based on the surrogate, the global sensitivity evaluations can be performed to evaluate the individual and collective influence of the kinematic parameters (design variables) and to order the relative importance of them in determining the aerodynamic outcome.

2.2.1 Design Space

The range of variables, see table 1, is comparable to that of a fruitfly and was chosen after considering the length and time scales observed in nature and compiled in references 54-56. Tabulated measurements of angular amplitudes and phase lags for a variety of species are not as well documented, and are selected in this study by matching the designated scaling parameters. As already stated, in this study, we focus on the Reynolds number of 100 under hovering conditions to establish a reasonable scope.

\begin{tabular}{ccc}
\hline \hline Parameter & Minimum & Maximum \\
\hline $2 h_{a} / c$ & 2.0 & 4.0 \\
$\alpha_{a}$ & $45^{\circ}$ & $80^{\circ}$ \\
$\varphi$ & $60^{\circ}$ & $120^{\circ}$ \\
\hline \hline
\end{tabular}

TABLE 1. Minimum and maximum values of the plunging amplitude ratio, angular amplitude, and phase lag that were evaluated.

\subsubsection{Design of experiments}

The number and the efficient distribution of the training points to populate the design space is considered thoroughly in the DOE. The DOE used a face centered cubic design (FCCD) ${ }^{45}$ and then Latin hypercube sampling (LHS) ${ }^{46}$ to appropriately fill in the remainder of the design space. The reasoning behind this is that a $2^{\text {nd }}$ order polynomial response surface construction has $(N+1)(N+2) / 2$ coefficients, $N$ being the number of variables, and in general, one wants twice this many data points for an initial curve fit which would try and reconcile the computational cost (fewer training points desired) with the resulting fidelity (more training points desired). A FCCD design provides $2^{N}+2 N+1$ points: $2^{N}$ corner points, $2 N$ face points, and one center point. Thus for three design variables, FCCD provides 15 of the 20 points required. The LHS then provides a method for efficiently choosing the rest of the points by maximizing the distance between the added points. A tabulation of the two-dimensional and three-dimensional simulations run and their respective outcomes, as well as quantitative measures of merit for the surrogate models, are found in Ref 43.

\subsubsection{Global sensitivity analysis}

The global sensitivity analysis (GSA) is in general useful for: (i) determining if a variable is particularly influential in the design space, if not perhaps the variable can be fixed and the degrees of freedom and complexity of the problem reduced; (ii) ranking the importance of the design variables; (iii) quantifying the degree of coupling between design variables. For example, 
is the influence on the design space mostly an individual effort, or is there an effect caused by the interaction of variables? For more details refer to Sobol's method ${ }^{46}$.

3. Results In this section, we use the surrogate modeling technique, utilizing the weight-averaged surrogate model ${ }^{51,52}$, to establish the relationship between lift generated and power required as the three flapping kinematic parameters vary. The purpose is to gain a global perspective of a multi-dimensional design space.

\subsection{Time-averaged Lift}

Figure 4 shows the surrogate models of the time-averaged lift, including those based on the two-dimensional and three-dimensional results, as well as their differences. Each axis corresponds to one of the design variables while the color contours illustrate the objective function of interest (e.g. time averaged lift or power). Qualitatively, the general trends found in two- and three-dimensional cases are consistent. As the angular amplitude is increased, a lower angle of attack results, causing a decreases of the lift. The general trend for plunging amplitude is also largely consistent between two- and three-dimensional cases. Alter the plunging amplitude and the resulting lift does not change significantly for much of the design space. There are regions in the design space where the time averaged lift shows clear differences between twoand three-dimensional cases. A closer inspection of the quantitative relationship between the lift and the kinematic parameters reveals that these differences can be substantial (illustrated in figure 4). These will be highlighted shortly.

To show the global impact of the kinematic variables in the entire design space Figure 5 illustrates the total variances due to the respective design variables. Immediately apparent is the change in the hierarchy of design variables. In two dimensions, the time-averaged lift was the most sensitive to the angular amplitude, significantly less sensitive to the phase lag, and even less still to the plunging amplitude. While the plunging amplitude was not a negligible influence, such an analysis can not only rank the relative importance but also illuminate variables which do have negligible influence. A finding of this nature can reduce the dimensionality of the design space greatly reducing the time it takes for refinement iterations because that variable can effectively be treated as a constant. In this study the plunging amplitude has been kept as a design variable for completeness.

In the three-dimensional case, figure 5 reveals both the plunging amplitude and the phase lag have substantially increased importance relative to the average lift produced compared to the two-dimensional case. So much so that the hierarchy of sensitivity changes from: 1) $\alpha_{a}$ 2) $\phi 3$ ) $h_{a}$ in two-dimensional, to three-dimensional where the order is 1) $\phi$ 2) $\alpha_{a}$ 3) $h_{a}$. In the present context the main and total variances are close enough to not warrant separate plots. This in turn implies a relatively small degree of coupling between the design variables and their resulting effect on the integrated lift. 


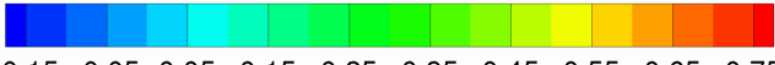

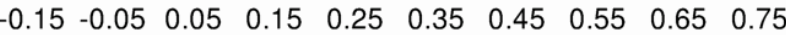
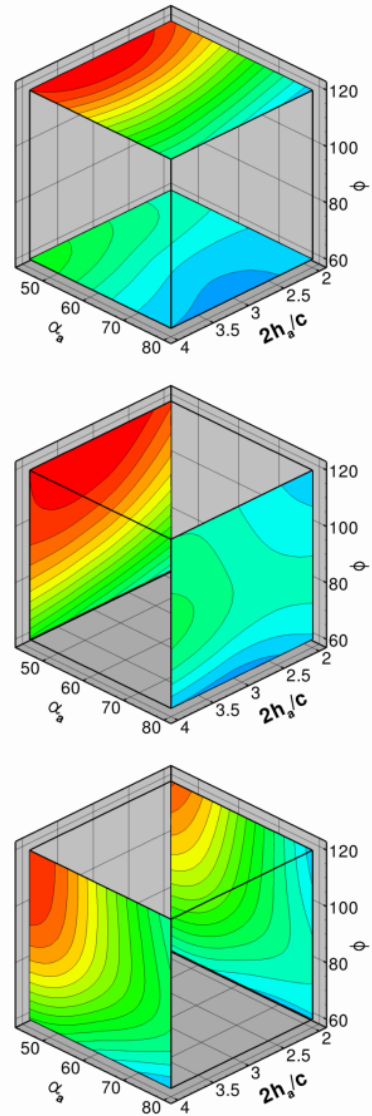

three-dimensional
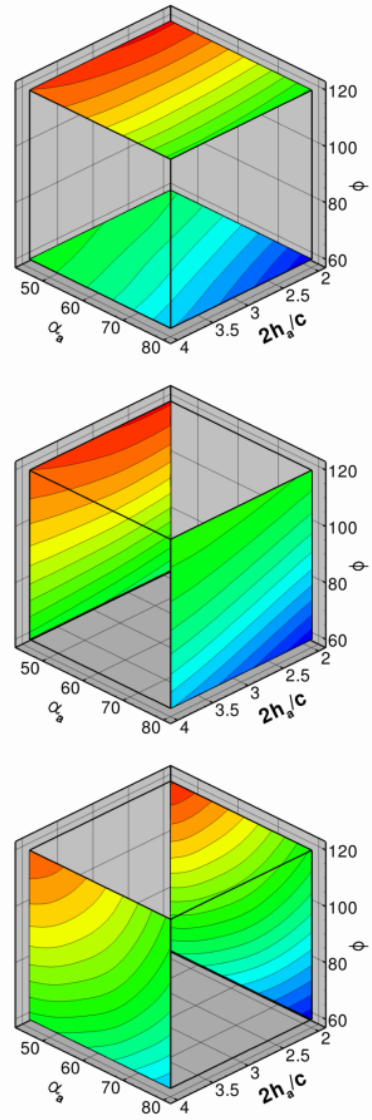

difference
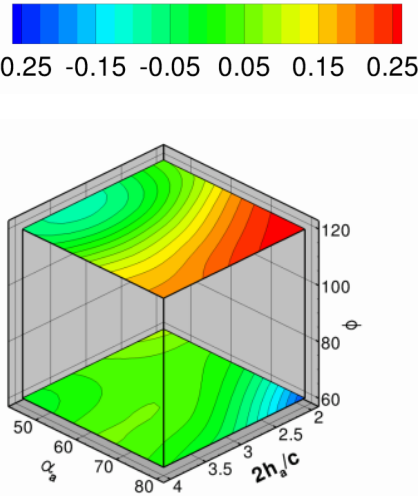

$\phi$

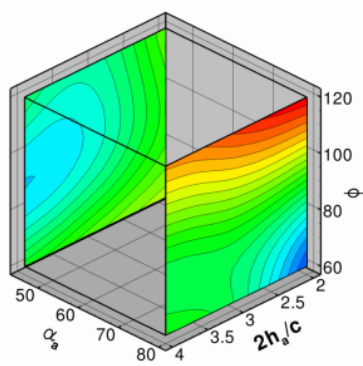

$\alpha_{a}$

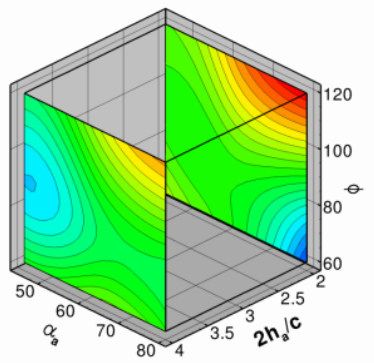

FIGURE 4. Surrogate modeling results for lift. Left- two-dimensional, Middle- three-dimensional, Right- three-dimensional minus the two-dimensional time averaged lift.
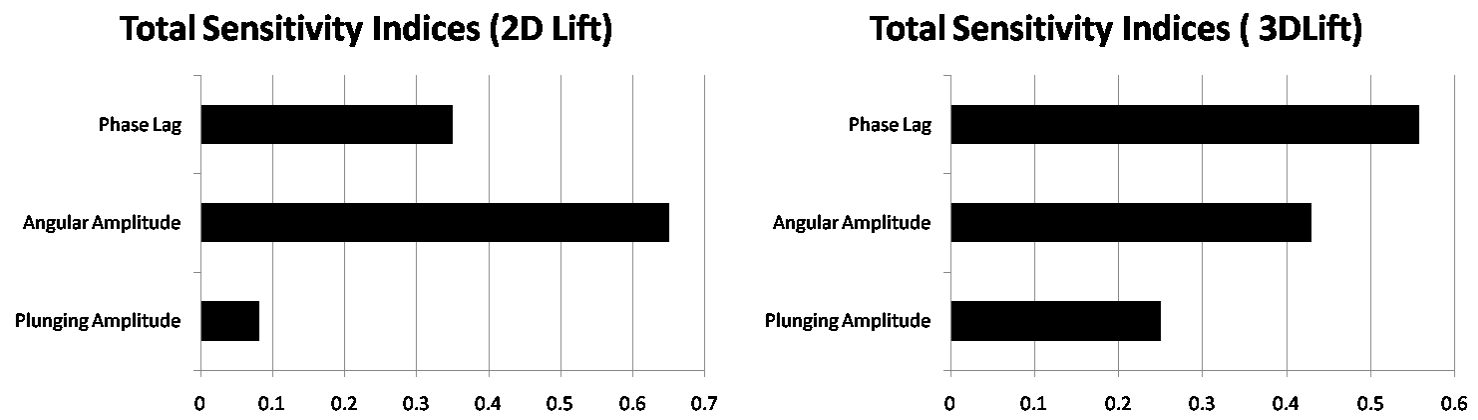

FIGURE 5. Global sensitivity analysis (GSA) of lift for two-dimensional and three-dimensional hovering kinematics. 
a

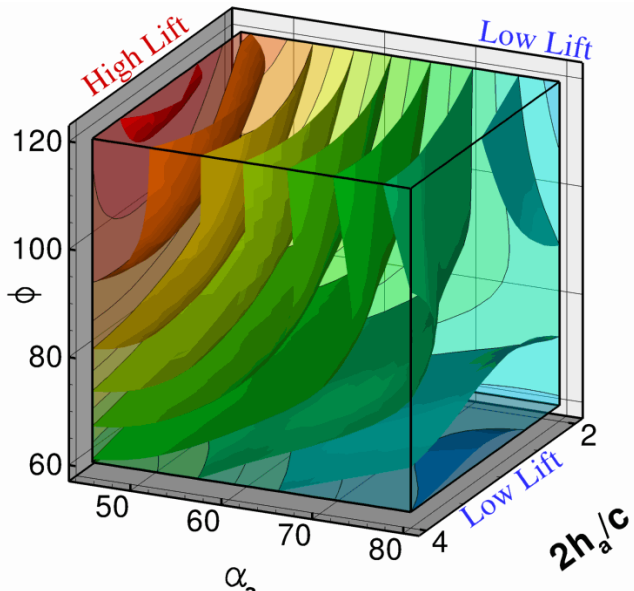

c

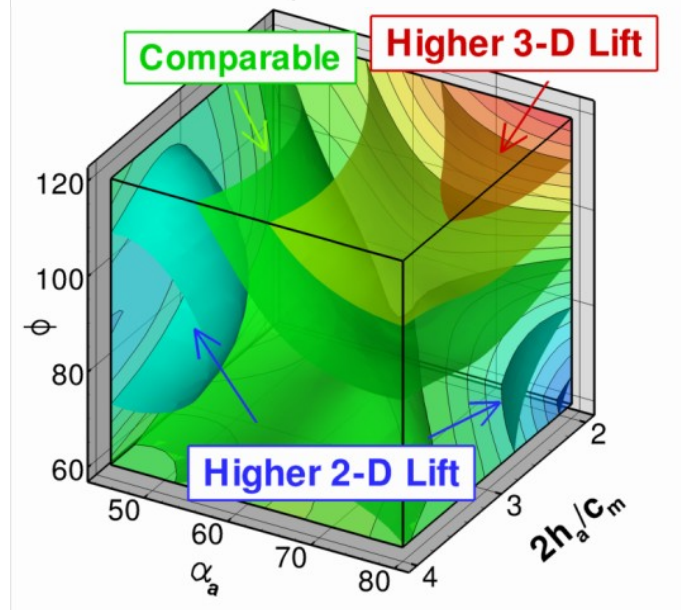

b
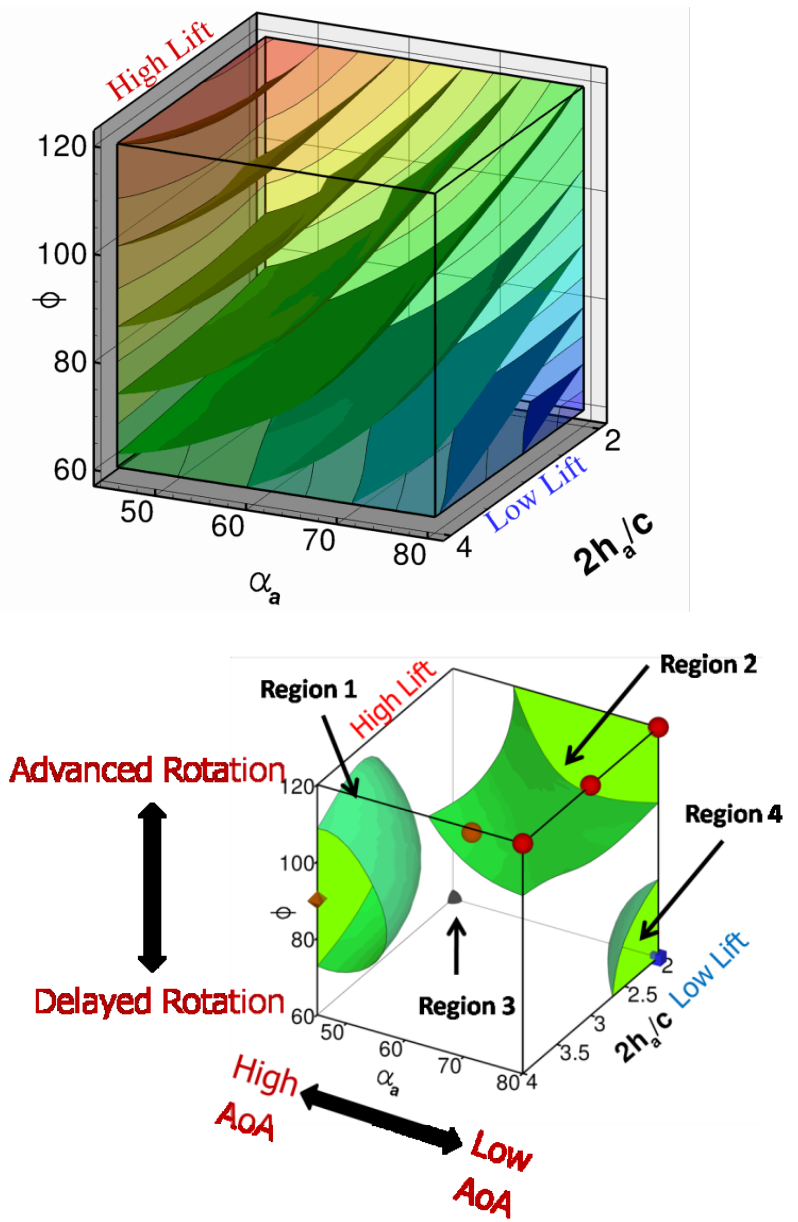

Figure 6. Iso-sufaces of 2D lift (a), 3D lift (b), 3D minus 2D lift (c), and (d) where the absolute difference between the 2D and 3D lift equals 0.10 . The symbols denote training points in those regions for which detailed force histories and flow field quantities are available; brown octahedra (region 1), circles (region 2), black quarter sphere (region 3), and a blue cube (region 4).

To identify where in the design space 3D effects become important, iso-surfaces of the absolute difference between the two and three-dimensional lift coefficients are shown in figure 6 . The green regions in the design space correspond to the kinematic parameters with the difference in time-averaged lift coefficient larger than 0.1 due to three-dimensionality. Four coherent regions are identified: region 1, characterized by synchronized hovering and low angular amplitude; region 2, with advanced rotation and high angular amplitude; region 3, with delayed rotation, low angular amplitude, and small plunging amplitude; and region 4, delayed rotation, high angular amplitude, and low plunge amplitude. Everywhere else the differences between the two- and three- dimensional lift is below the 0.1 threshold. The similarities in this catch-all region are defined by the likeness of their time averaged values, but in some instances, as will be shown, stronger statements may be appropriate.

\subsection{Region 1; Synchronized Hovering, High AoA}

Region 1 is defined by kinematics that are close to synchronized hovering (i.e. including cases with slight delayed or advanced rotation), low angular amplitude (high AoA), and larger plunging amplitudes. The time history of lift, see figure 7 , shows two local maxima per stroke in 
2D. The first peak is associated with the wake capture at the beginning of the stroke, see figure 8 at $t / T=0.8$. Between the two peaks is a local minimum referred to as a wake valley which is caused by a combination of decreasing angle of attack and interaction with a pocket of downward momentum. For this region of the 2D kinematic design space, this pocket of downward momentum takes the form of a persistent jet. As reported during the experimental studies of Freymuth (1990), the jet develops as a result of a reverse Karman vortex street interacting with the downward momentum created by the wing as it translates. As the wing passes the jet, vortices are shed with an orientation that reinforces the downward momentum previously created by the wing, see figure 9 . These vortices sustain the downward momentum, and further entrain surrounding fluid, as to create a flow feature which the wing then interacts with during subsequent stroke. After this the LEV is the dominate flow feature (see figure 8 at $t / T=1.0$ ) with a significant impact on the lift (figure 7).

Figures 8 and 9 show significant noticeable variation in the flow physics encountered for the finite wing. A useful quantity when examining vortical structures is the $Q$-criterion ${ }^{53}$, the second invariant of the velocity gradient tensor, defined as,

$$
Q=-\frac{1}{2} \frac{\partial u_{i}}{\partial x_{j}} \frac{\partial u_{j}}{\partial x_{i}}=-\frac{1}{2}\left(S_{i j} S_{i j}-\frac{1}{2} \omega_{i} \omega_{i}\right),
$$

where $S_{i j}$ is the rate of strain tensor, and $\omega_{i}$ is the vorticity vector, both in indicial notation.

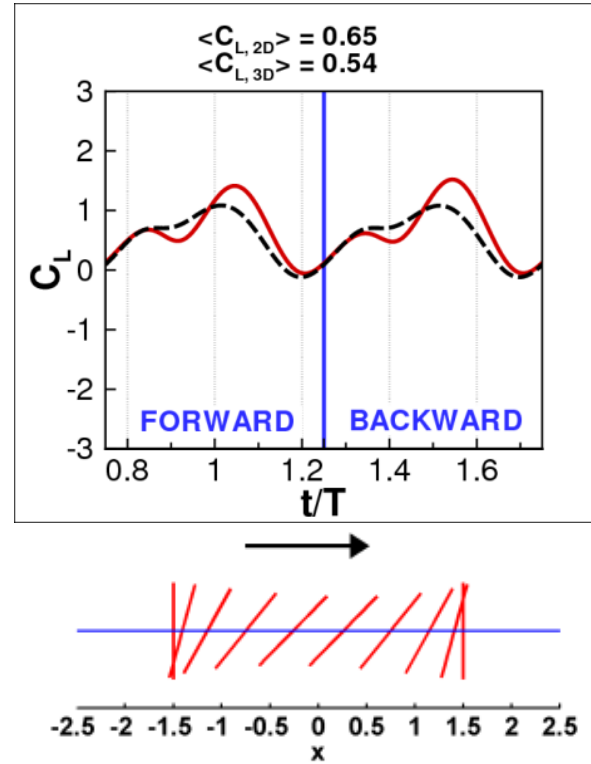

Figure 7. Lift coefficients, 2D (solid red) and 3D (dashed black), of a representative case in region $1,2 h_{a} / c=$ 3.0, $\alpha_{a}=45^{\circ}$, and $\phi=90^{\circ}$ (case 11).

Figure 9 shows an iso- $Q$ contour colored by $\omega_{3}$, the spanwise-vorticity. In this fashion we can separate the rotation from the shear, via $Q$, and then get directional information with vorticity. The vortices shed from the leading from the leading and trailing edges identified by red and blue colors respectively, while the tip vortex is left green. The role of the tip vortex in the hovering cases studied is particularly interesting. 


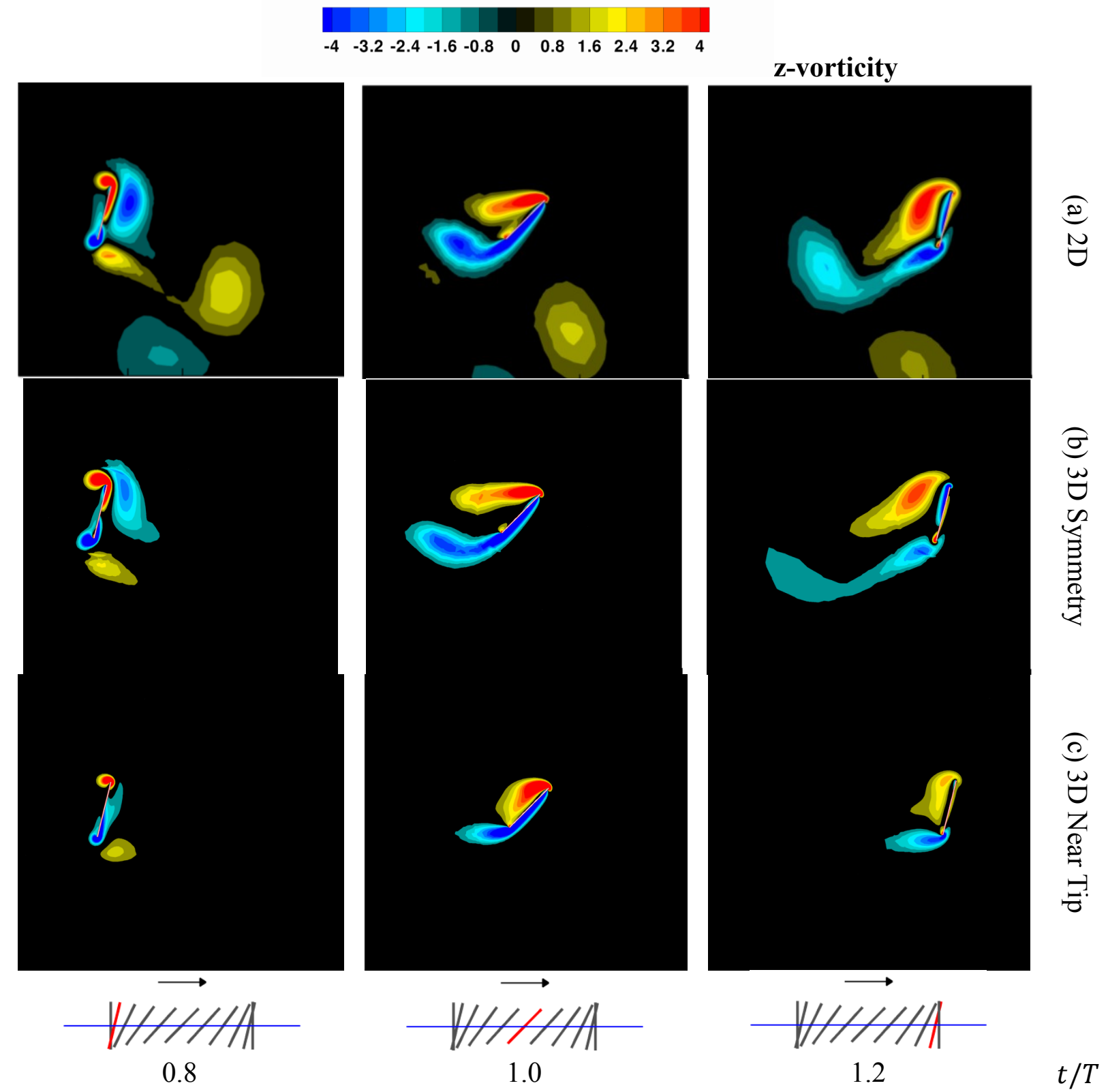

FIGURE 8. Z-vorticity contour plots at three time instants in the forward stroke, i.e. $t / T=0.8,1.0$, and 1.2, for the case $11\left(h_{a}=3.0, \alpha_{a}=45^{\circ}, \phi=90^{\circ}\right)$ : (a) from $2 \mathrm{D}$ computation; (b) in the symmetry plane of 3D computation; (c) near the wingtip $(\mathrm{z} / \mathrm{c}=1.8)$ plane from the $3 \mathrm{D}$ computation.

In general the tip vortices are found to have four prominent competing effects: TE1) Enhancement of lift due to the proximity of the associated low pressure region of the tip vortex next to the airfoil, TE2) Induced downwash acting to reduce the effective angle of attack along the span weakening the LEV hence reducing the instantaneous lift, TE3) Interaction with the vortices shed from the leading and trailing edges anchoring them from shedding near the wing tips enhancing the lift (region 3), TE4) Due to the tip vortices pulling fluid from the underside of the wing to the upper side, the wing leaves behind a weaker pocket of downward momentum in 
the flow. Upon interaction with this downward momentum, a loss in lift is seen and so weaker wake-valley means higher lift.

In the three-dimensional case the weaker downward momentum (TE4) pocket does help compared to $2 \mathrm{D}$, see $t / T=0.9$ in figure 7 . Figure 9 shows the jet-flat plate interaction in both dimensions. In $2 \mathrm{D}$ the persistent jet shows larger $u_{2}$ magnitudes, and is narrower. However the weaker LEV (TE2) as shown in figure 8, causes the lift to suffer. In the two-dimensional case the LEV is largely attached and the benefit of anchoring the LEV is not helping. The overall impact for this case is that the two-dimensional lift, $\left\langle C_{L, 2 \mathrm{D}}\right\rangle=0.65$, is better than the threedimensional counterpart, $\left\langle C_{L, 3 \mathrm{D}}\right\rangle=0.54$. More generally, cases with kinematics in region 1 have larger lift in $2 \mathrm{D}$.
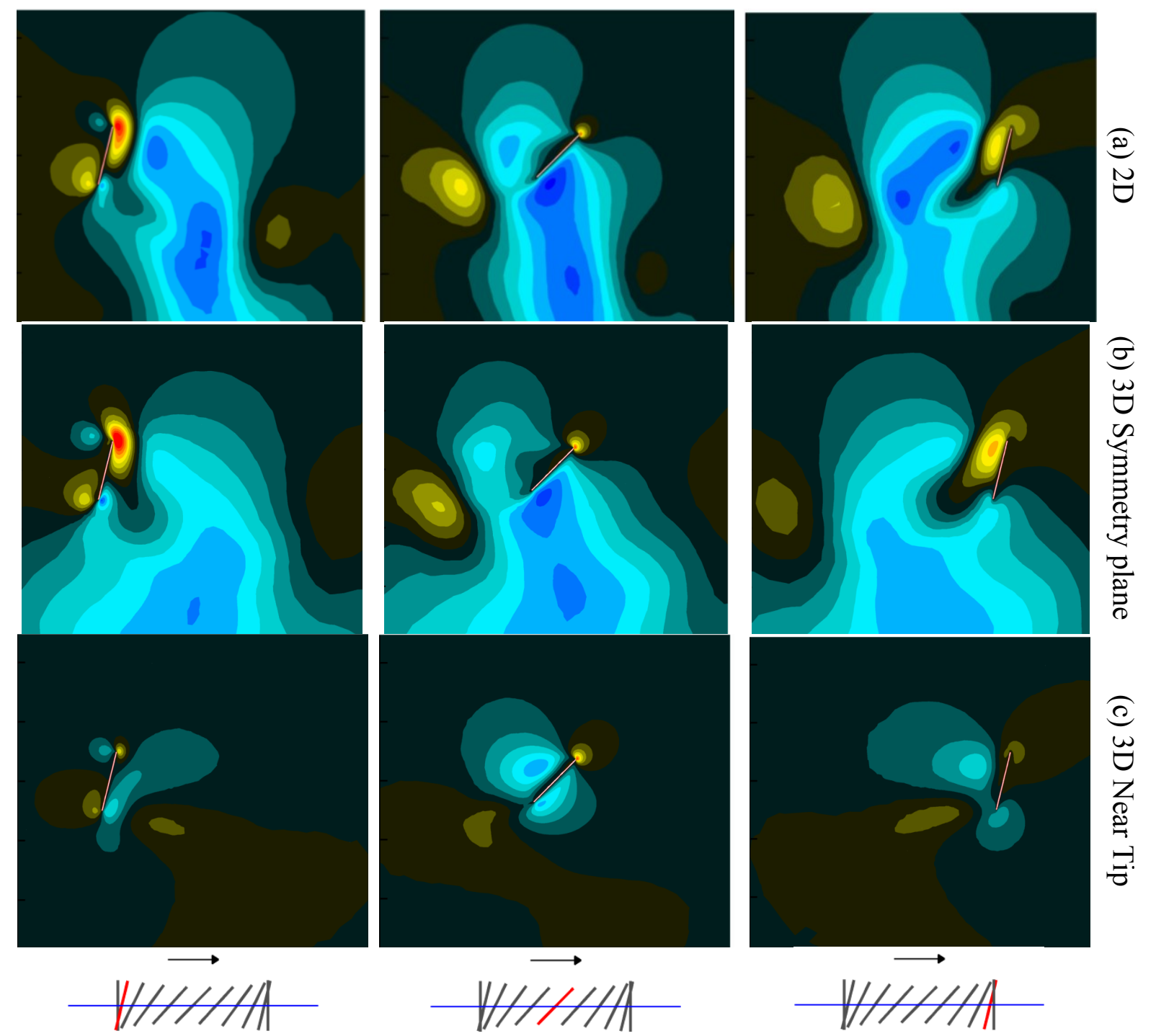

0.8

1.0

1.2

$t$
$/ T$

FIGURE 9. Vertical velocity contour plots at three time instants in the forward stroke, i.e. $t / T=0.8,1.0$, and 1.2, for the case $11\left(h_{a}=3.0, \alpha_{\mathrm{a}}=45^{\circ}, \phi=90^{\circ}\right)$ : (a) from two-dimensional computation; (b) in the symmetry plane of three-dimensional computation; (c) near the wingtip $(z / c=1.8)$ plane from the three- 


\section{dimensional computation.}

\section{Q-criterion}

\section{Spanwise Lift}

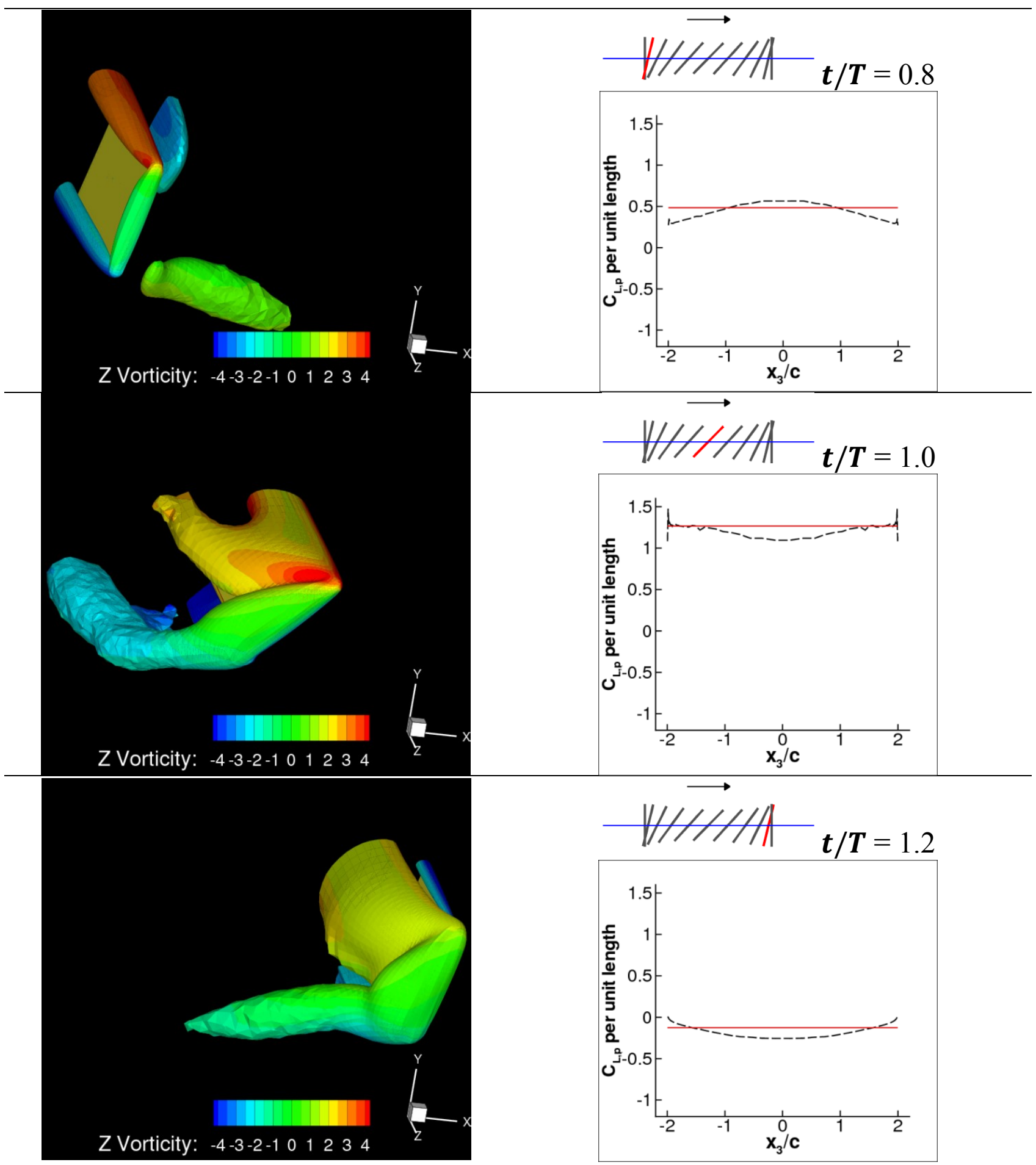

Figure 10. The lift per unit span and iso- $Q$ surfaces $(Q=0.75)$ colored by $z$-vorticity over half of the wing using the kinematic parameters $2 h_{a} / c=3.0, \alpha_{a}=45^{\circ}, \phi=90^{\circ}$ (case 11) at $R e=100$ at $t / T=0.8,1.0,1.2$. The spanwise variation in forces is examined with the two-dimensional equivalent marked for reference. 


\subsection{Region 2; Advanced Rotation, Low AoA}

In region 2 the kinematics are characterized by advanced rotation, and high angular amplitude. Figure 11 shows the time histories of lift and the associated flow features from case 8 $\left(2 h_{a} / c=4.0, \alpha_{a}=80^{\circ}\right.$, and $\left.\phi=120^{\circ}\right)$ which is representative for the other cases in region 2.

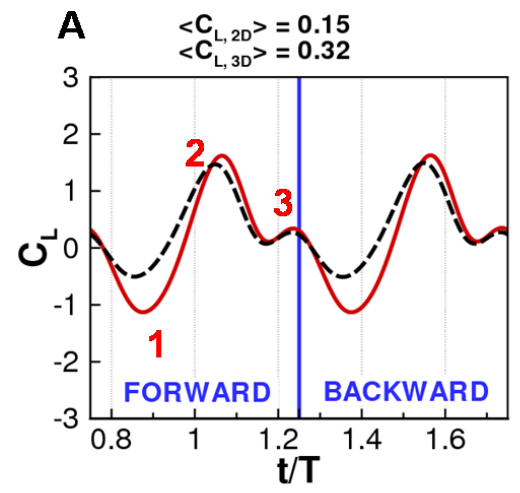

B

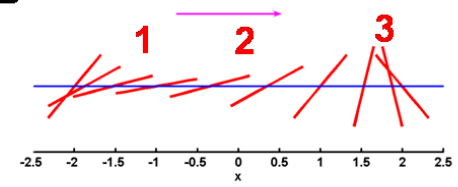

1.

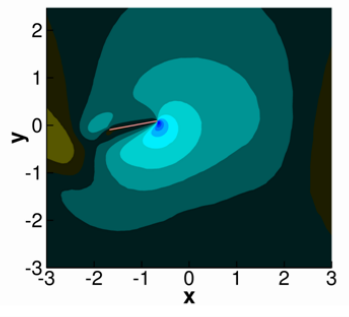

$\mathrm{u}_{2}$

2.

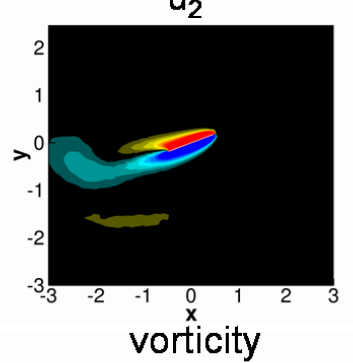

3.

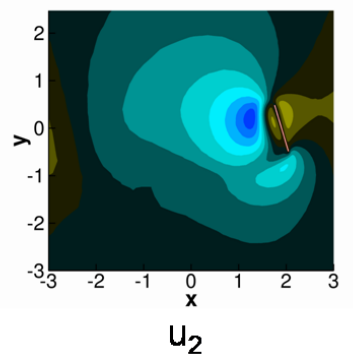

Figure 11 Time history of lift coefficients in a representative case in region $1,2 h_{a} / c=4.0, \alpha_{a}=80^{\circ}$, and $\phi=$ $120^{\circ}$, with the associated flow features. (A) Lift, $\left(C_{L}\right)$, during a motion cycle. Red-solid, two-dimensional computation; black-dashed, three-dimensional computation. (B) kinematic schema of the flat plate motion. (C) Representative flow features at 1$) t / T=0.9, u_{2}$ contours; 2 ) $t / T=1.0$, vorticity contours; 3 ) $t / T=1.2, u_{2}$ contours.

Right after the stroke reversal, the flat plate moves into the wake generated in the previous stroke. Due to the downwash in this wake, see figure $11 \mathrm{C} 1$, and low and decreasing angle of attack (figure 11B), lift drops (Note: the the pocket of downward momentum encountered for these kinematics is not a persistent jet). The three-dimensional case does not suffer the same drop in lift as the two-dimensional case. 


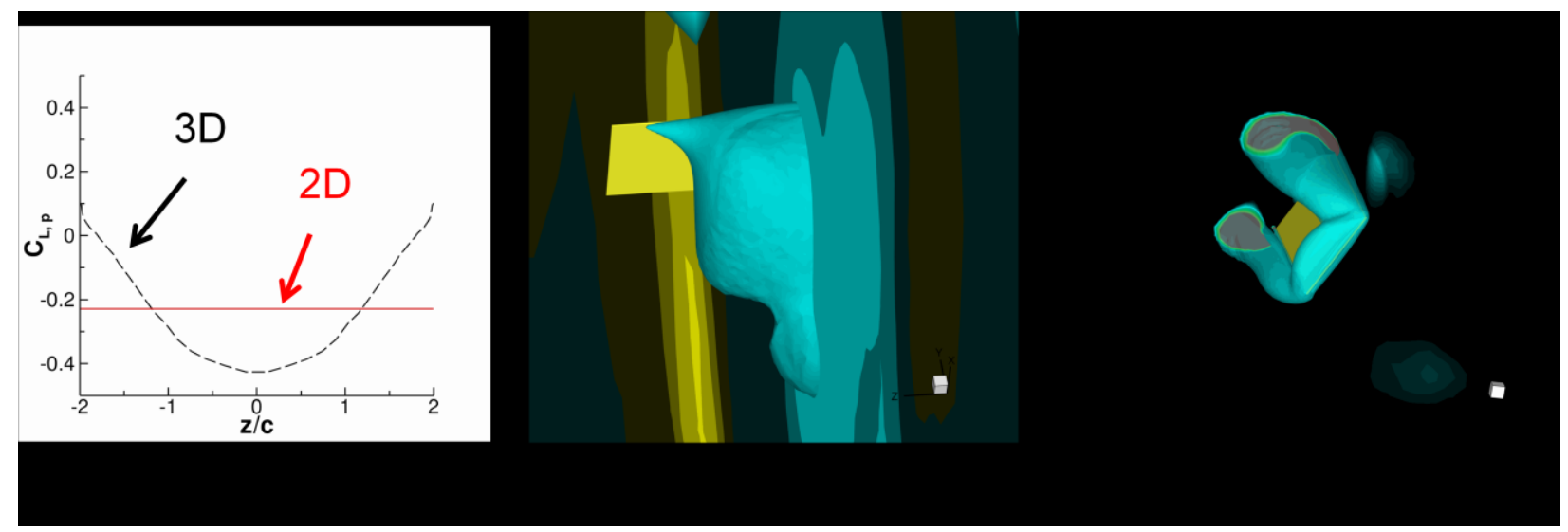

Figure 12 (A) The lift per unit span; (B) Iso-surfaces of vertical velocity $\left(u_{2}=-0.3\right)$; $($ C) Iso-surfaces of $Q(Q=$ 4). Case 8: $2 h_{a} / c=4.0, \alpha_{a}=80^{\circ}$, and $\phi=120^{\circ}$ at $\mathrm{t} / \mathrm{T}=0.9$.

\subsection{Region 3; Delayed Rotation, High AoA, Low Plunging Amplitude}

In classical steady state theory the presence of wing tip vortices is attributed to a loss of lift and increase in drag when comparing the two-dimensional wing to its finite three-dimensional counterpart $^{57}$. What is seen, in the current study, are instances in which low aspect ratio, unsteady aerodynamics are producing tip vortices which can enhance lift while negligibly influencing the drag (shown in section 3.7).

Region 3 is defined by kinematics with delayed rotation, low angular amplitude (high AoA), and shorter plunging amplitudes. This region shows a significant impact from the tip vortices. Figure 13 presents a delayed rotation case with $2 h_{a} / c=2.0, \alpha_{a}=45^{\circ}, \phi=60^{\circ}$. The difference in the flow physics encountered due to three-dimensional phenomena is noticeable. The main characteristics of the vortices, including sizes, strengths and movement are distinctly different between two- and three-dimensional results. Not only is there a strong spanwise variation in the three-dimensional flow, but also there is little resemblance between the symmetry plane of the three-dimensional computation and the two-dimensional computations.

In two-dimensional flow, the pair of the large scale vortices are noticeably closer to each other and the airfoil than that in the three-dimensional flow. The instantaneous lift coefficient for the two cases examined is illustrated in 14 , depicting noticeable differences in that the threedimensional lift coefficient is generally higher than its two-dimensional counterpart. With these kinematics patterns the tip vortices can interact with the LEV to form a lift enhancement mechanism. This aspect will be discussed next.

For the case presented in figure 15 (delayed rotation), the $Q$ iso-surface colored with $\omega_{3}$ vorticity, along with the spanwise distribution of $C_{L}$, due to pressure, the effects of the tip vortices become apparent. Firstly, there is a low pressure region at the wing tip favorably influencing the lift (TE1). Furthermore, the tip vortex anchors the large scale vortex pair near the tip (TE3). At midspan, however, the vortex pair has separated from the wing. This in turn drives the spanwise variation seen in the flow structures and force history. 

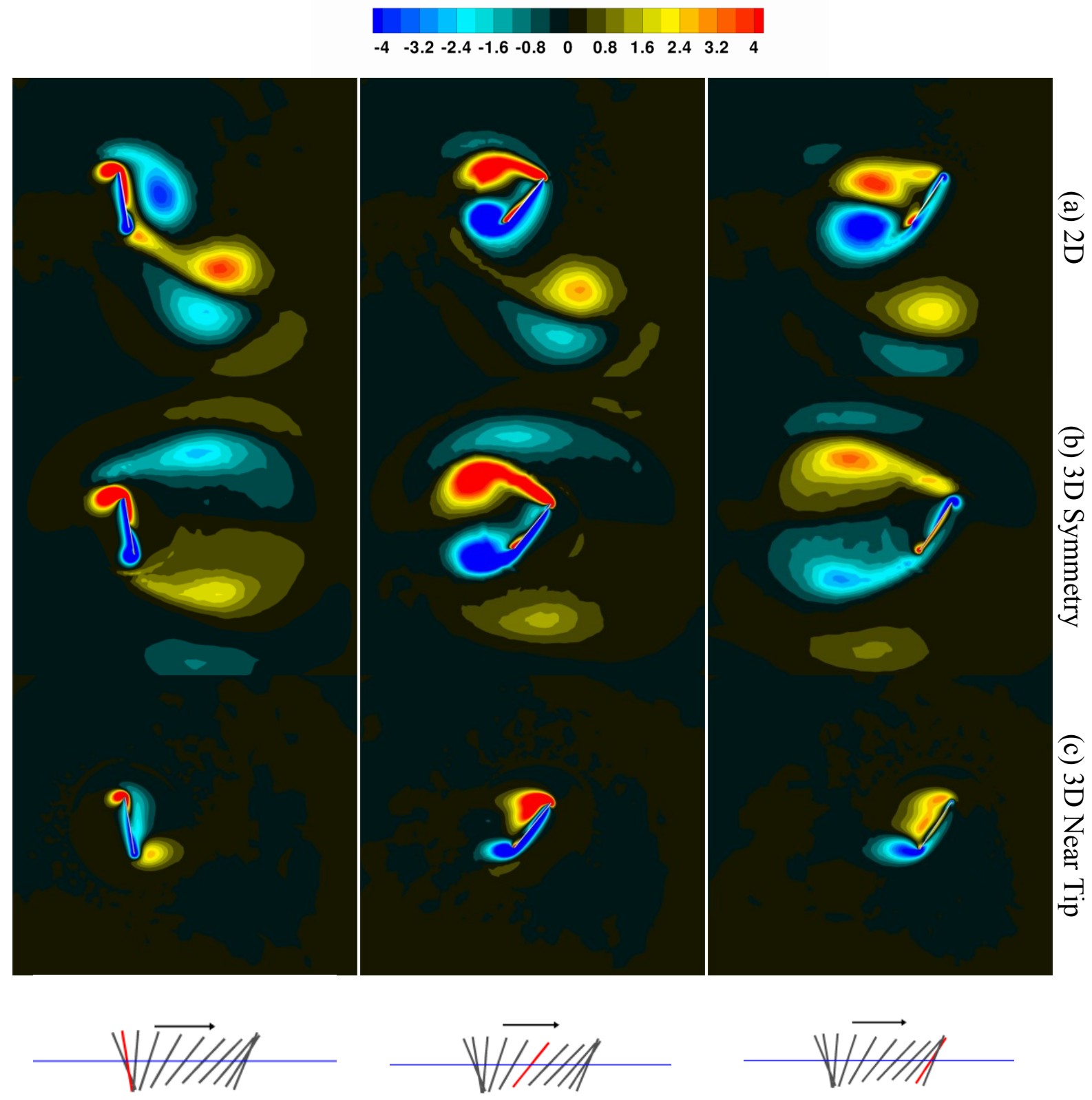
0.8
1.0
1.2
$t$
$/ T$

FIGURE 13. Z-vorticity contour plots at three time instants in the forward stroke, i.e. $t / T=0.8,1.0$, and 1.2, for the case $1\left(h_{a}=2.0, \alpha_{a}=45^{\circ}, \phi=60^{\circ}\right)$ : (a) from two-dimensional computation; (b) in the symmetry plane of three-dimensional computation; (c) near the wingtip $(z / c=1.8)$ plane from the three-dimensional computation. 


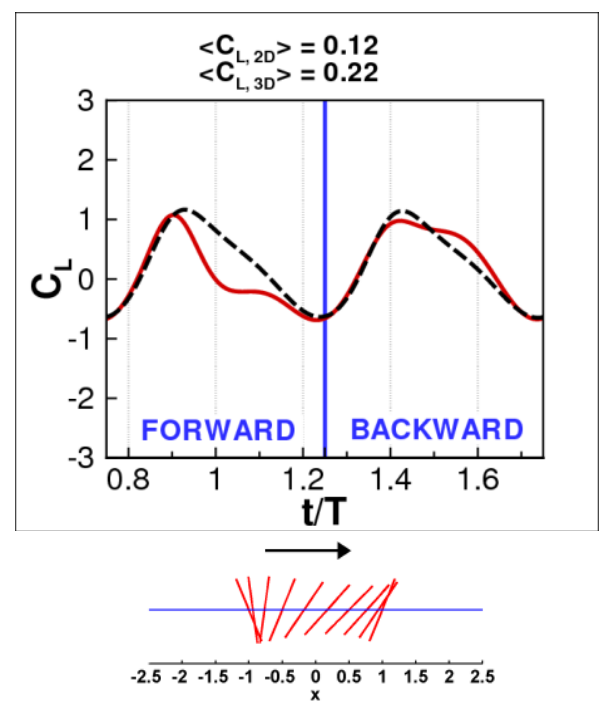

Figure 14. Time history of lift coefficients in a representative case in region $3,2 h_{a} / c=2.0, \alpha_{a}=45^{\circ}$, and $\phi=$ $6^{\circ}$ (case 1), with red-solid $=2 \mathrm{D}$; black-dashed $=3 \mathrm{D}$.

Compared to an infinite wing, the tip vortices caused additional mass flux across the span of a low aspect-ratio wing, which helps push the shed vortex pair, from the leading and trailing edges, at mid-span away from one another. Furthermore, there is a spanwise variation in effective angle of attack induced by the downwash (TE2), stronger near the tip. Overall, the tip vortices allowed the vortex pair in their neighborhood of the tip to be anchored near the wing surface, which promotes a low pressure region and enhances lift. The end result is an integrated lift value that departs from the two-dimensional value considerably.

It is clear that the kinematic motions have a significant impact on tip vortex formation and the leading/trailing edge vortex dynamics. Interestingly, for many of the kinematic motions examined, the tip vortex force enhancement could be confined to lift benefits, i.e. the resulting drag did not increase proportionally! 


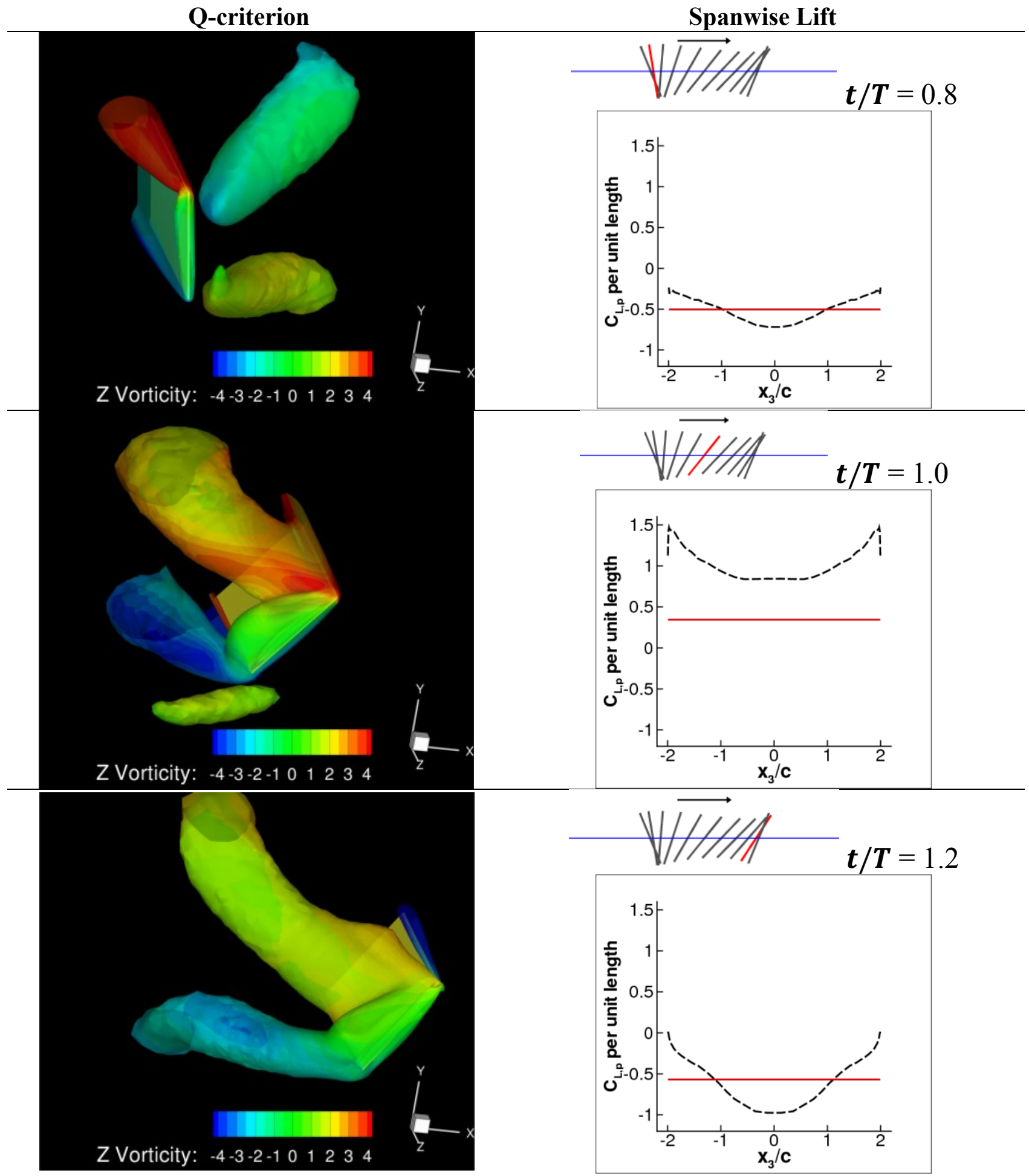

FIGURE 15. The lift per unit span and iso- $Q$ surfaces $(Q=0.75)$ colored by $z$-vorticity over half of the wing using the kinematic parameters $2 h_{a} / c=2.0, \alpha_{a}=45^{\circ}, \phi=60^{\circ}$ (case 1) at $R e=100$ at $t / T=0.8,1.0,1.2$. The spanwise variation in forces is examined with the two-dimensional equivalent marked for reference.

Time averaged lift coefficient for i) two-dimensional: 0.13 , ii) three-dimensional: 0.22 . 


\subsection{Region 4; Delayed Rotation, Low AoA, Low Plunging Amplitude}

Region 4 is defined by kinematics with delayed rotation, large angular amplitude (or low AoA), and shorter plunging amplitudes. Figure 16 shows the time histories of lift from the twodimensional, and the three-dimensional computations along with a schema for the kinematics: $2 h_{a} / c=2.0, \alpha_{a}=80^{\circ}$, and $\phi=60^{\circ}$ as a representative case for this region. The largest discrepancy between two-dimensional and three-dimensional is seen around $t / T=0.9$. Because the rotation is delayed, after the stroke reversal at $t / T=0.75$ the flat plate creates rotational starting vortices to increase the lift, with its first peaks around $t / T=0.9$. However, as shown in figure 17, in the two-dimensional case, the TEV shed in the previous stroke interacts with the flat plate after the stroke reversal enhancing the lift by the wake-capture mechanism $(t / T=$ $0.9)$. On the other hand, for the three-dimensional case, the shed LEV and the TEV, repel from each other and the path of the flat plate, such that after the stroke reversal, the wake-capturing is absent. The first lift peak in the time history in figure 16 is then only due to the rotational effects. So the diverging behavior of the vortices, observed for all delayed rotation cases, and the interaction of the vortices from the LE, and TE with the wing tip vortex, play a central role as important three-dimensional effects as described in Region 3.

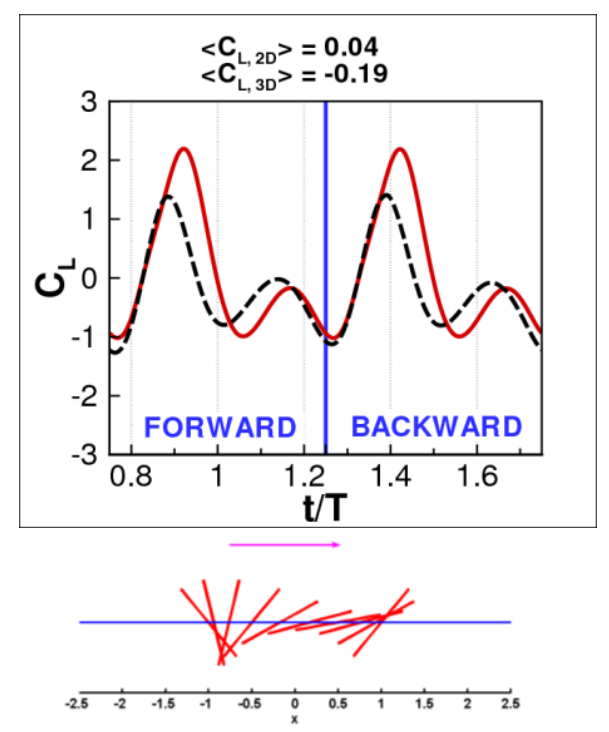

Figure 16. Time history of lift coefficients in a representative case in region $2,2 h_{a} / c=2.0, \alpha_{a}=80^{\circ}$, and $\phi=$ $60^{\circ}$ (case 3), with red-solid=two-dimensional; black-dashed=three-dimensional. 


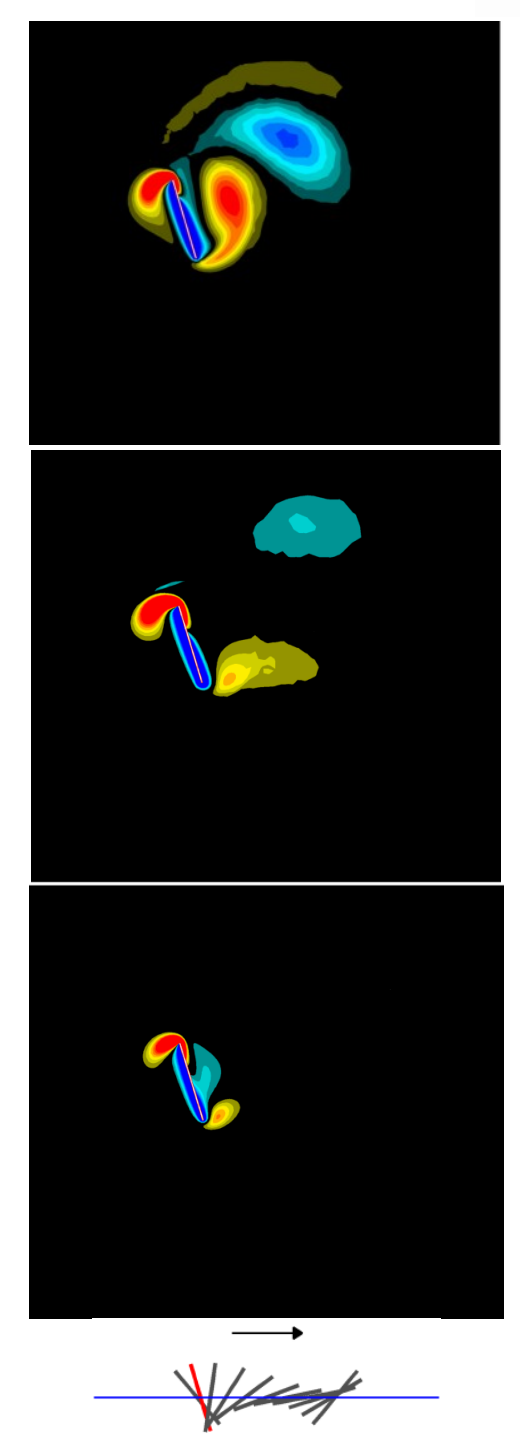

0.8
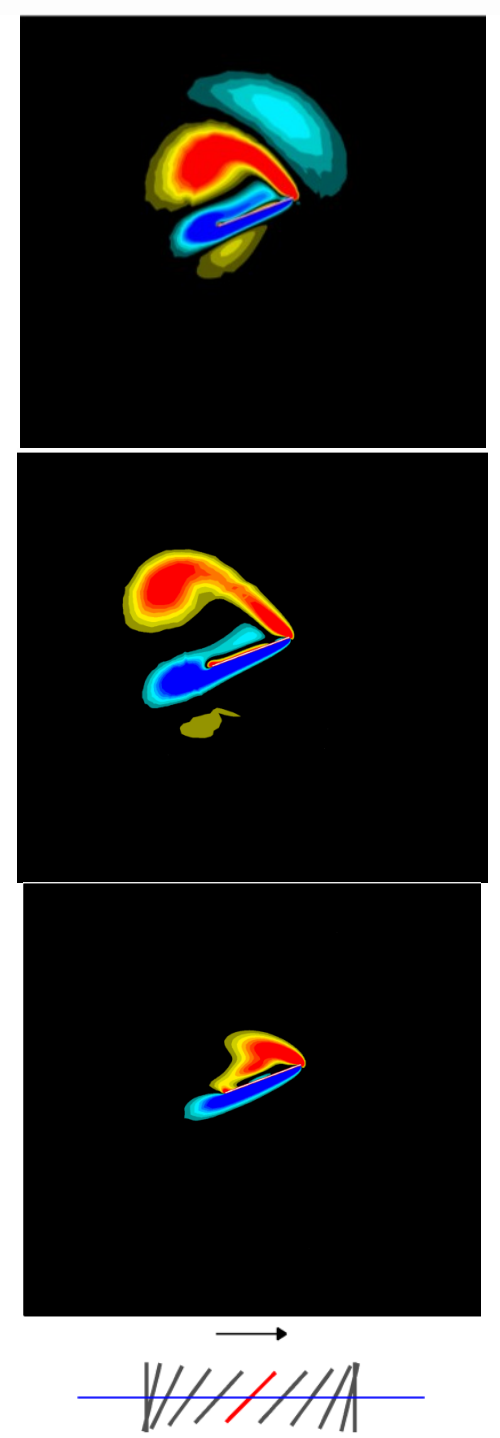

1.0

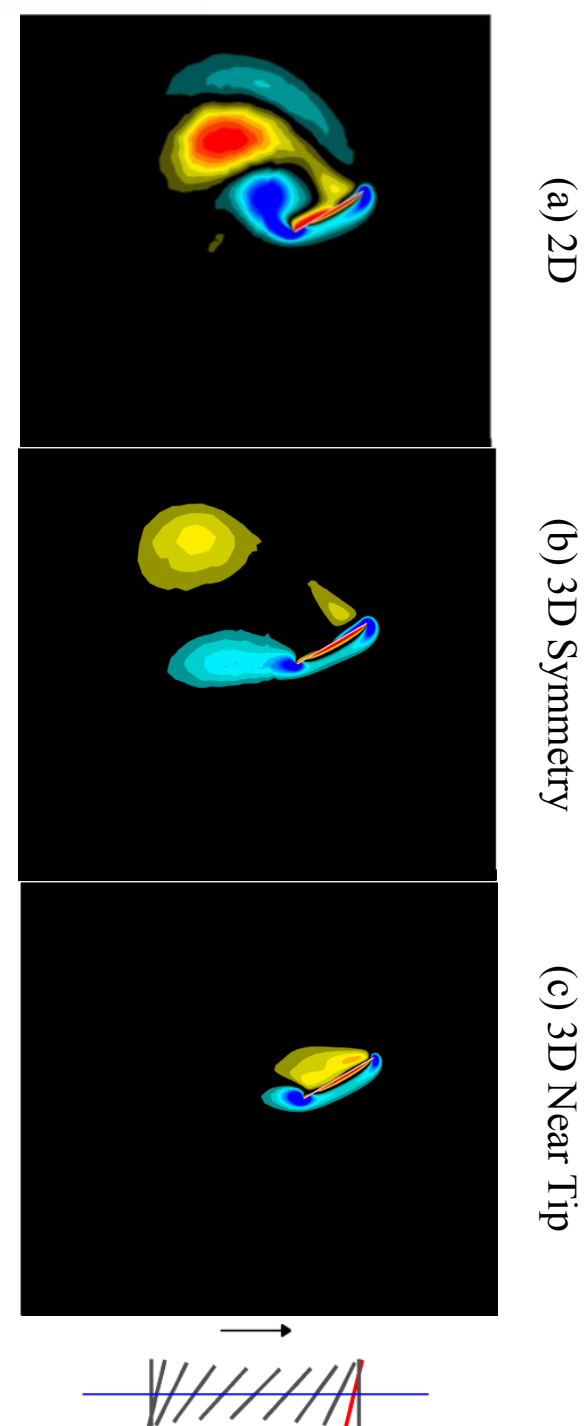

1.2

FIGURE 17. Z-vorticity contour plots at three time instants in the forward stroke, i.e. $t / T=0.8,1.0$, and 1.2 , for the case $3\left(h_{a}=2.0, \alpha_{a}=80^{\circ}, \phi=60^{\circ}\right)$ : (a) from 2D computation; (b) in the symmetry plane of 3D computation; (c) near the wingtip $(\mathrm{z} / \mathrm{c}=1.8)$ plane from the 3D computation.

\subsection{Region of similarity}

For other kinematic combinations, the integrated forces over time match reasonably, i.e. the difference between the two-dimensional, and the three-dimensional time averaged lift is less than 0.1 . For some cases even the instantaneous forces agree closely: a synchronized rotation case with low angles of attack is remarkably similar when examining two-dimensional and threedimensional force histories, see figure 18. 


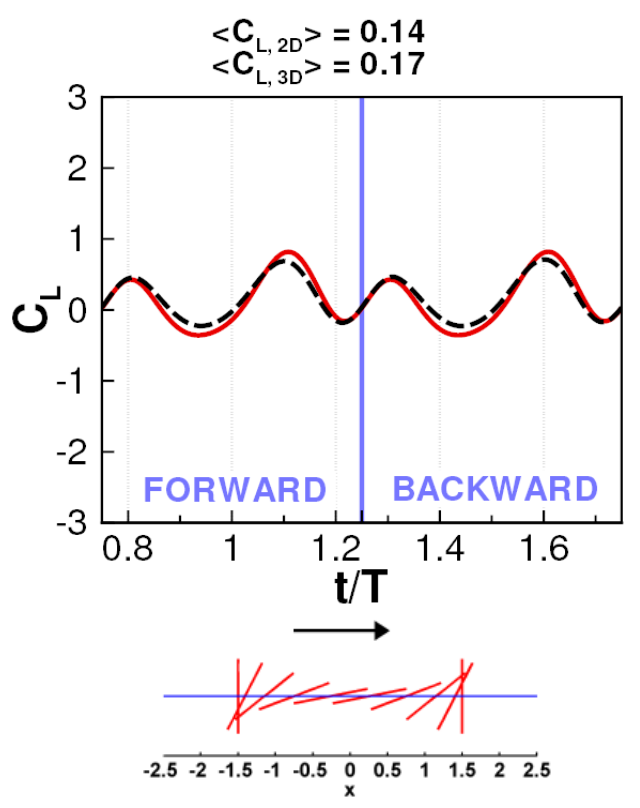

Figure 18. Time history of lift coefficients for a case in the region of similiarity, $2 h_{a} / c=3.0, \alpha_{a}=80^{\circ}$, and $\phi=$ $\mathbf{9 0}^{\circ}$ (case 12), with red-solid=two-dimensional; black-dashed $=$ three-dimensional.

Figure 19 shows the flow fields corresponding to the parameters: $2 h_{a} / c=3.0, \alpha_{a}=$ $80^{\circ}, \phi=90^{\circ}$. The variation along the spanwise direction is modest, making the two-dimensional and three-dimensional simulations substantially similar. The two-dimensional flow field and the corresponding three-dimensional flow on the symmetry plane are strikingly consistent. The high angular amplitudes lead to low angles of attack, and coupled with the timing of the rotation, leads to a flow that does not experience delayed stall as the formation of the LEV is not promoted. The timing of the rotation for this example puts the flat plate at its minimum angle of attack at maximum translational velocity, while the translational velocity is zero when the flat plate is vertical.

As previously remarked, neither the two-dimensional or three-dimensional results in this case promote downward jet formation. As summarized in figure 18, the two-dimensional and threedimensional lift coefficients of this case are similar in the instantaneous as well as the time averaged sense. One implication illustrated is the usefulness of two-dimensional simulations for quantitative data on a three-dimensional counterpart.

Figure 20 shows a $Q$ contour and the associated spanwise lift distribution. It is seen from the flowfield that the tip vortex as well as the leading and trailing edge vortex formation is largely suppressed. The angles of attack are relatively low during the maximum translational velocities, approximately $10^{\circ}$, and the translational velocities are small when the wing approaches $90^{\circ}$ near the ends of the strokes. The net effect is a fairly uniform spanwise lift distribution closely resembling the two-dimensional case with the same kinematics. 


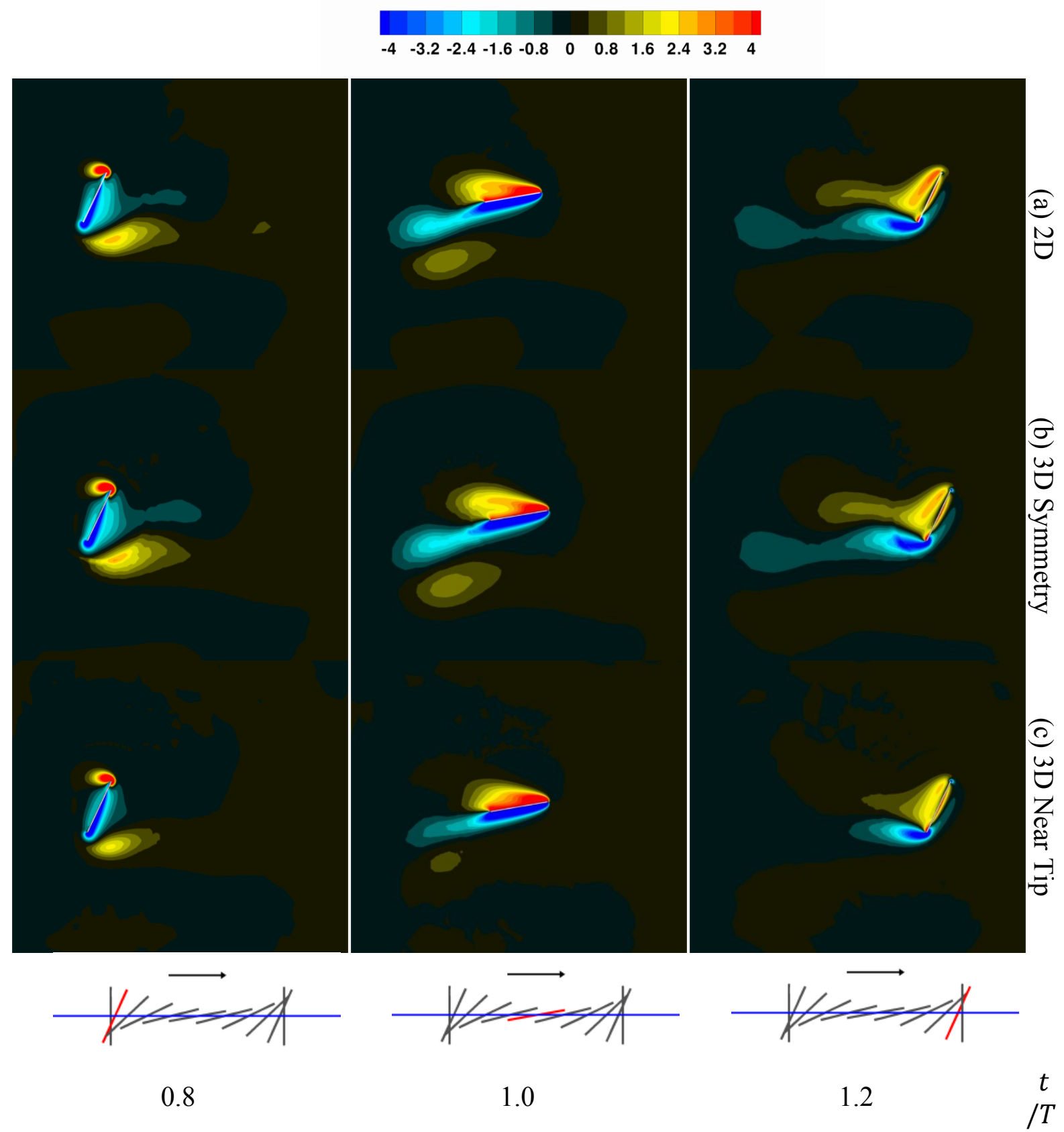

FIGURE 19. Z-vorticity contour plots at three time instants in the forward stroke, i.e. $t / T=0.8,1.0$, and 1.2, for the case $12\left(2 h_{a} / c=3.0, \alpha_{a}=80^{\circ}, \phi=90^{\circ}\right)$ : (a) from 2D computation; (b) in the symmetry plane of 3D computation; (c) near the wingtip $(z / c=1.8)$ plane from the 3D computation. 


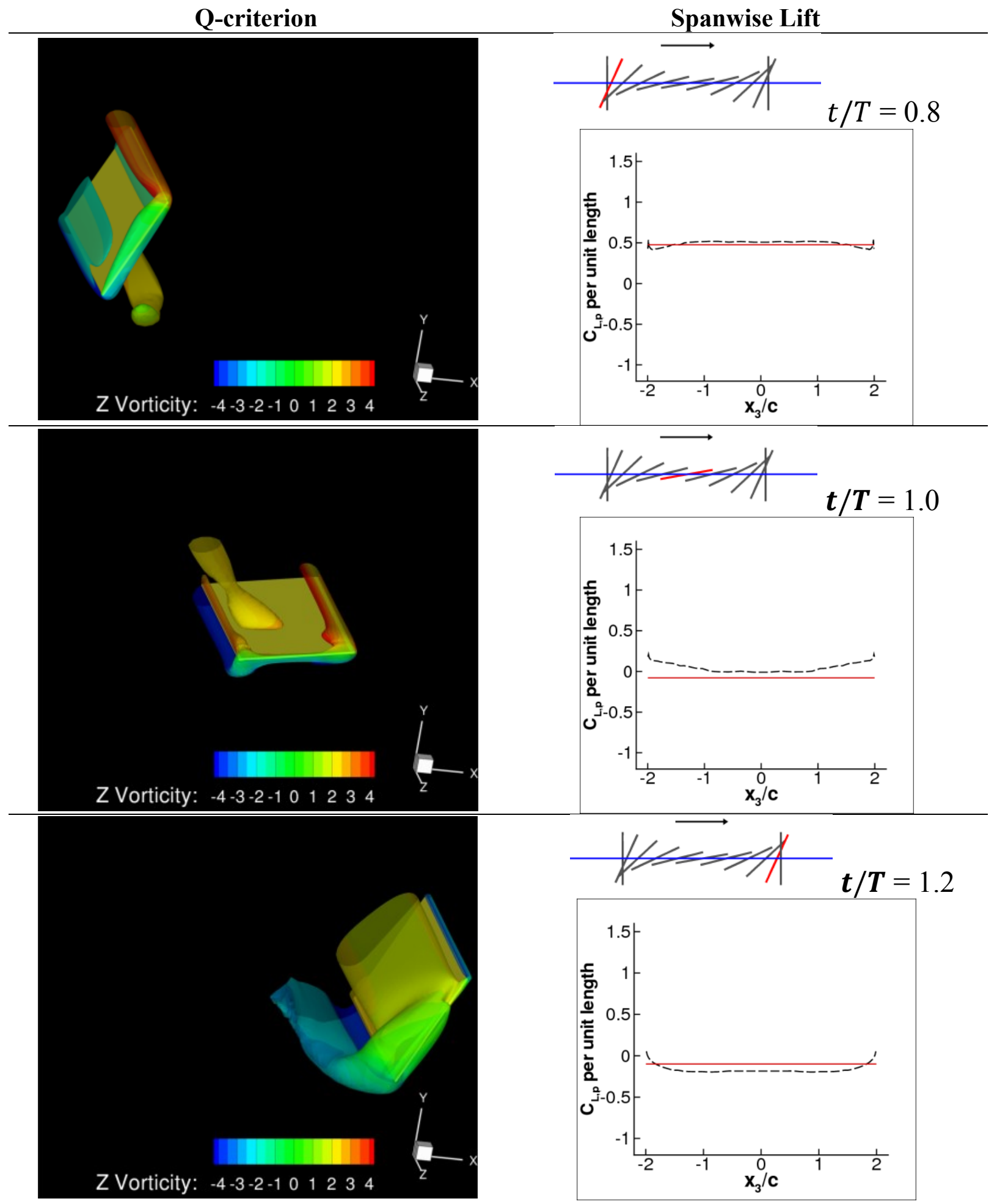

FIGURE 20. The lift per unit span and iso- $Q$ surface $(Q=0.75)$ colored by $z$-vorticity for flow fields over half the wing associated with the kinematic parameters $2 h_{a} / c=3.0, \alpha_{a}=80^{\circ}, \phi=90^{\circ}$ at $R e=100$ at $t / T=0.8$, 1.0, 1.2. The spanwise variation is limited. Time averaged lift coefficient for i) two-dimensional: 0.14 , ii) threedimensional: 0.17 .

3.7. Power Requirements 
Figure 21 displays the two-dimensional and three-dimensional surrogate responses of the power required, estimated by multiplying the pressure force by the instantaneous translational velocity and integrating over a cycle, as well as their differences. Not only are all the trends quite similar but the magnitudes also compare quite well (note the broader range of the contours seen for the power required often yields less significant differences). This implies that certain threedimensional flow features, not observable/possible in two-dimensional flows, for which lift was sensitive to, do not play a significant role in determining the drag for these kinematic combinations. This is an interesting consequence which warrants further study. From the instantaneous force histories, see appendix A, the agreement in drag coefficients is very close except at a combination of low plunging amplitudes with low angular amplitudes and low phase lags. Figure 22 measures the sensitivity of the power required to the kinematic variables. The hierarchy remains the same from $2 \mathrm{D}$ to $3 \mathrm{D}$, unlike in lift.

two-dimensional
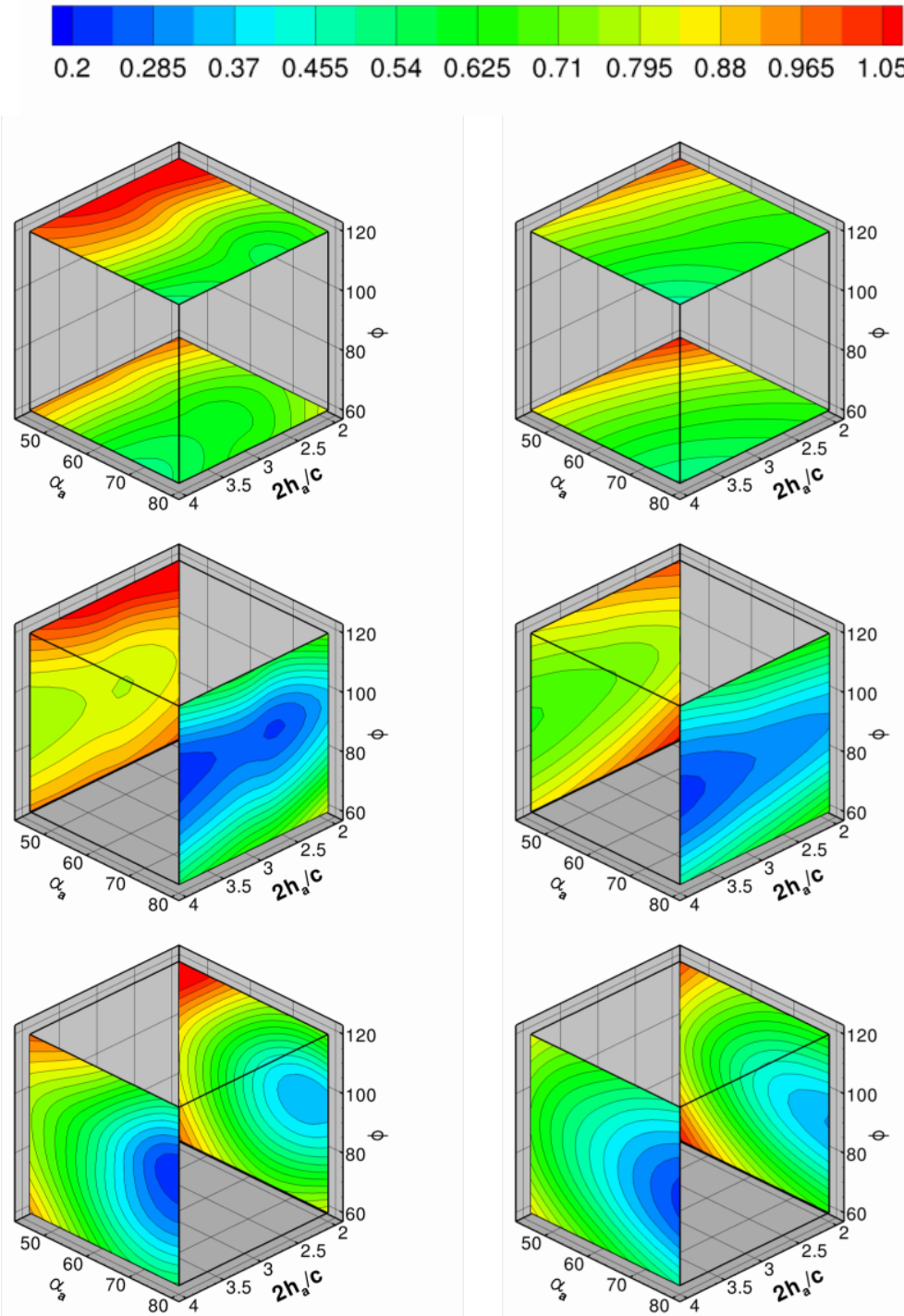

three-dimensional
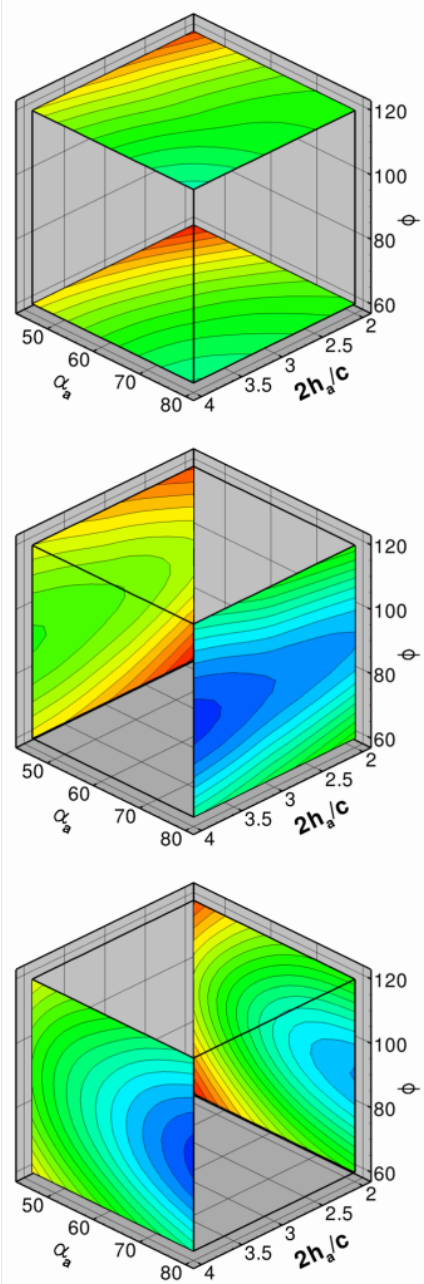

difference
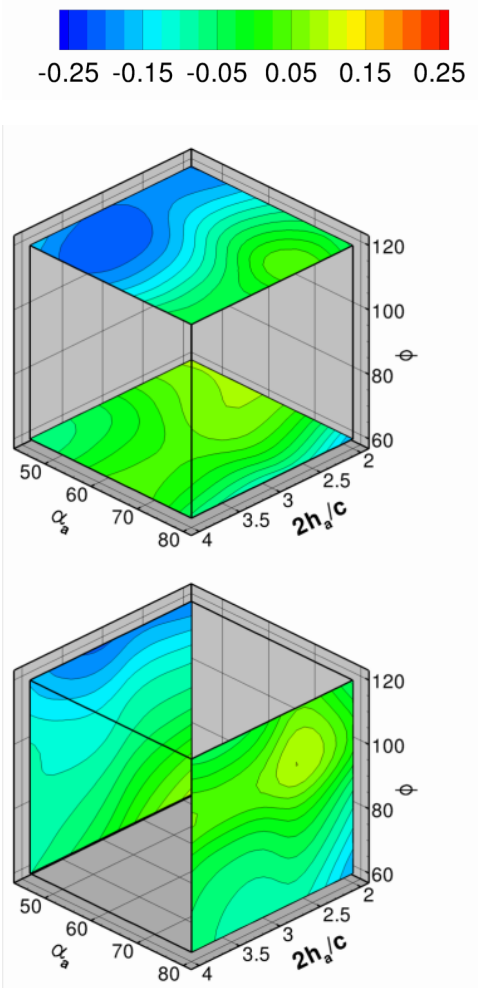

$\alpha_{a}$

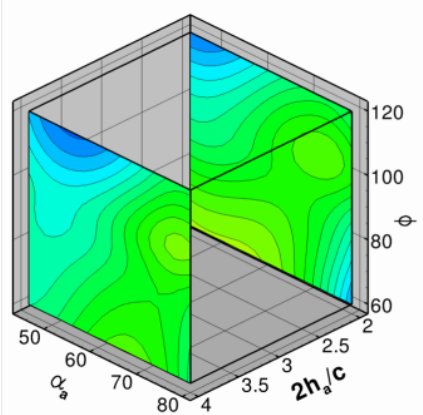

FIGURE 21. Surrogate modeling results for power required. Left- two-dimensional, Middle-threedimensional, Right- three-dimensional minus two-dimensional time averaged power requirement approximations. 


\section{Total Sensitivity Indices (2D Power)}

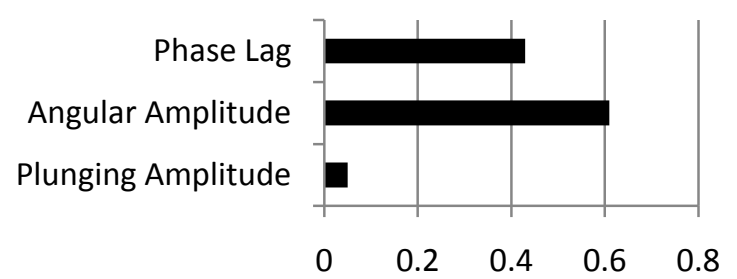

\section{Total Sensitivity Indices (3D Power)}

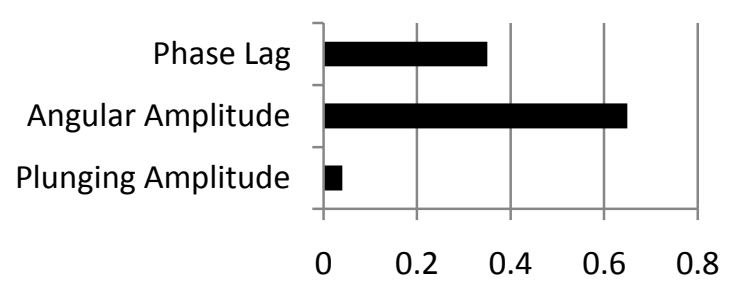

Figure 22. Global sensitivity analysis (GSA) of power for two-dimensional and three-dimensional hovering kinematics.

The physical reasoning behind the observed trends in two-dimensional and three-dimensional is the same, in that the agreement is not merely a consequence of integration. During normal hovering, $\phi=90^{\circ}$, the airfoil/wing is perpendicular to the direction of motion at the ends of translation but has little translational velocity. As the phase lag is perturbed in either direction the airfoil can have an appreciable velocity, while perpendicular to the direction of motion and whether the body is accelerating when perpendicular, as in delayed rotation cases, or decelerating, as in advanced rotation cases has a sizable impact on the instantaneous drag that is felt (see appendix B), and consequently the power required for the maneuver. Quality measures of the surrogate models are tabulated in reference 43. Compared to the differences between surrogates for lift, the differences for power between two- and three-dimensional cases are smaller overall, with a total variation of $30 \%$.

\subsection{Pareto front}

In a multi-objective investigation, it is often the case that different goals are in competition regarding suitable selections of design variables. One tool used to evaluate the trade-offs between objective functions is called the Pareto front ${ }^{58}$. The Pareto front consists of nondominated points and can be thought of as the set of best possibilities, as illustrated in figure 23 . Non-dominated points can be thought of as points for which one could not improve all objective functions simultaneously. The current objectives are to maximize lift and minimize power requirement. Points on the Pareto front therefore involve those for which increases in lift are accompanied by increases in power, and vice versa. To pose all objectives as minimization expressions, any objective which is maximized, like lift, is multiplied by a negative sign. Note that the Pareto front itself is very comparable between two and three dimensions. The primary differences are the peak lift values attained in two-dimensional flow exceeding their threedimensional counterparts, and the fact that the density of design variable combinations near the front is higher in the three dimensional case. The paths through the design space are plotted below their respective Pareto fronts in figure 23. Note: the jaggedness of the path is due to the resolution of the tested points and is seen because of the fine balance in objective functions for design variables in that region. It is seen that the high lift region follows the lower bound of the angular amplitude suggesting that future iterations should decrease the lower bound for higher lift solutions. Overall the design variable combinations on the optimal front are consistent qualitatively. 
As shown in figure 4, and a representative case illustrated in figure 24 , the highest timeaveraged lift values are obtained by a combination of advanced rotation and low angular amplitude in two and three dimensions. The general trends present, i.e. when holding two of the input variables constant and varying the last one, remained largely the same. For power required, figure 21 shows that the synchronized rotation cases with high angular amplitude consume the least power for both two-dimensional as well as three-dimensional hovering. The combination of lower angle of attack during the mid-stroke when the translational velocity is at maximum and small translational velocities at the ends of the strokes when the flat plate is vertical minimizes the power. These trends not only followed similar qualitative trends between two-dimensional and three dimensional cases, but also consistently quantitatively matched in magnitude.

two-dimensional
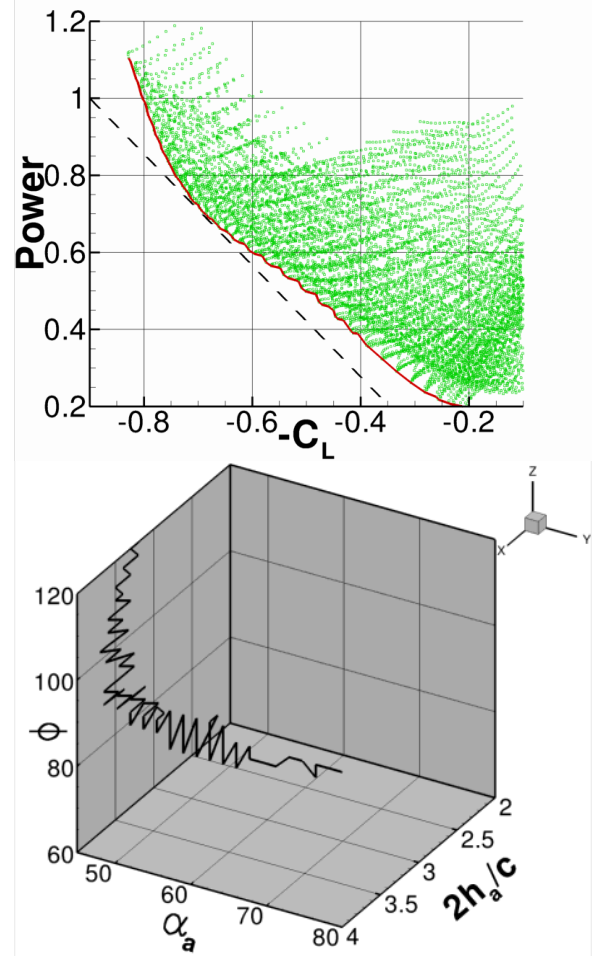

three-dimensional
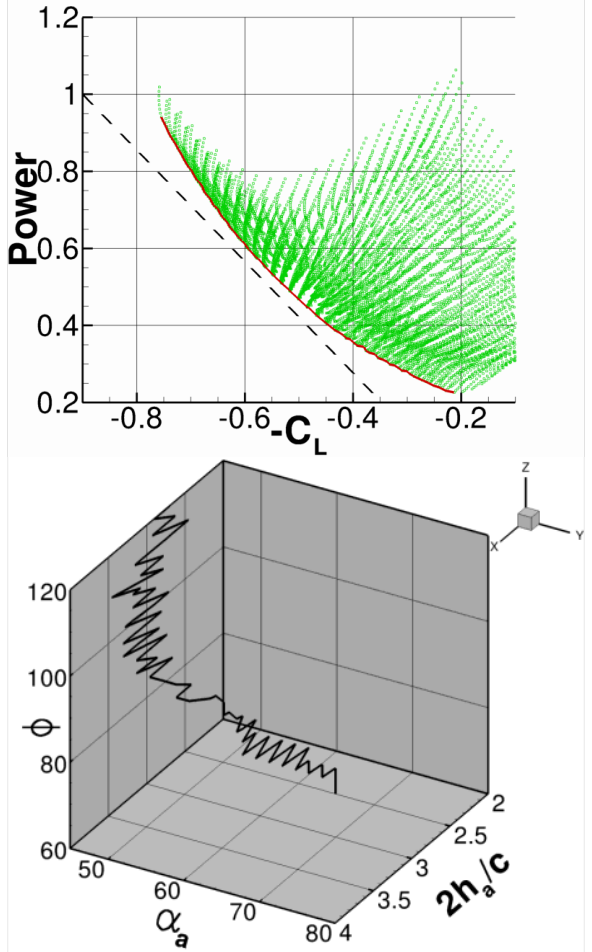

FIGURE 23. Pareto fronts illustrating the competing objectives of lift and power requirements in twodimensions (left) and three-dimensions (right) and the design variable combinations which provide those fronts. The dashed line is for reference.
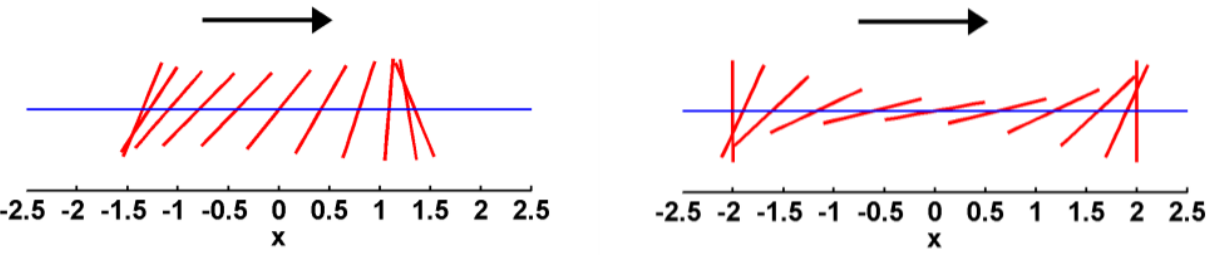

Figure 24. Representative high lift (left: $2 h_{a} / c=2.7, \alpha_{a}=45^{\circ}$, and $\phi=120^{\circ}$ ) and low power (right: $2 h_{a} / c=$ 4.0, $\alpha_{a}=80^{\circ}$, and $\phi=90^{\circ}$ ) kinematics.

\subsection{Environmental Sensitivity}

One of the main difficulties in realizing a functional MAV is its inherent sensitivity to the operating environment due to its size and weight. While the vehicle dynamics are going to be sensitive to the environmental perturbations, due to the fact that the flapping frequencies are an 
order or two magnitude greater than the wind gusts, $\mathrm{O}(10) \mathrm{Hz}$ to $\mathrm{O}(100) \mathrm{Hz}$ versus wind at $\mathrm{O}(1)$ $\mathrm{Hz}$, the aerodynamics associated with flapping wings can be pragmatically modeled by a constant freestream. A study was carried out using different kinematic schemes, freestream strengths, and freestream orientations. The three kinematic patterns chosen were those of sections 3.2, 3.4, and 3.6. These patterns were chosen due to having pronounced LEVs (3.2), beneficial tip vortices (3.4), and negligible 2D vs 3D differences (3.6) respectively. The freestream strength varied from $5 \%-200 \%$ of the maximum translational velocity. If one were comparing this to fruitflies, (wingspeed $\sim 3.1 \mathrm{~m} / \mathrm{s}$ ) the freestream would range from $0.15-6.2$ $\mathrm{m} / \mathrm{s}$. The directions of the freestream varied between heading down, right, or up.

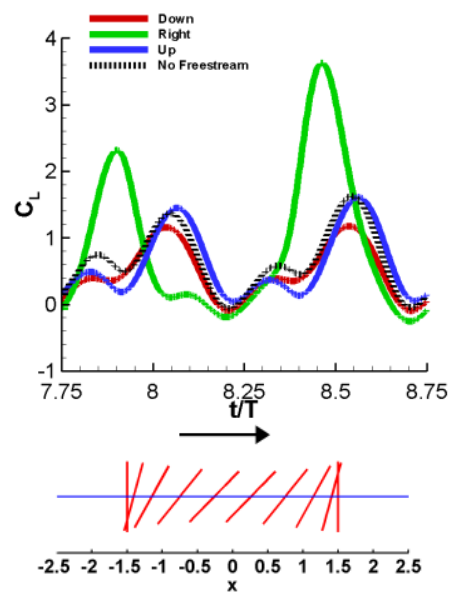

a) $2 h_{a} / c=3.0, \alpha_{a}=45^{\circ}$, and $\phi=90^{\circ}$

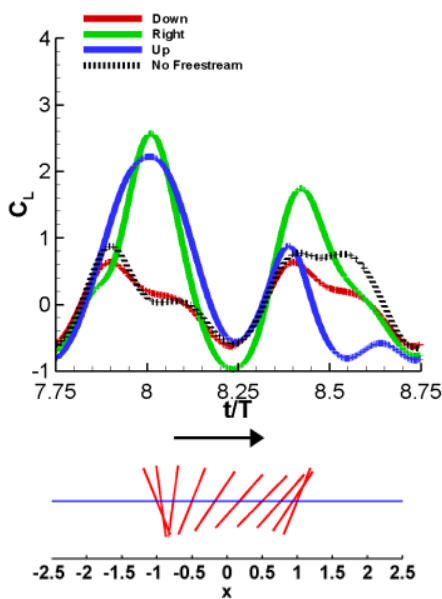

b) $2 h_{a} / c=2.0, \alpha_{a}=45^{\circ}$, and $\phi=60^{\circ}$

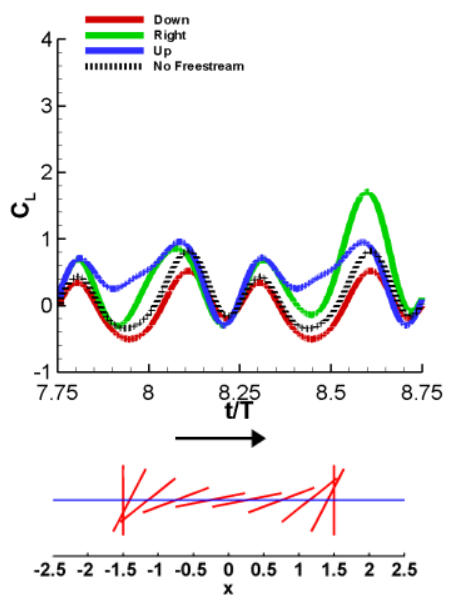

c) $2 h_{a} / c=3.0, \alpha_{a}=80^{\circ}$, and $\phi=90^{\circ}$

Figure 25. Two-dimensional $C_{L}$ in response to a freestream with a magnitude of $20 \%$ of the maximum plunging velocity heading in three distinct directions (down, right, and up) for three hovering kinematics.

The 2D cases were much more sensitive to the freestream than their 3D counterparts which will be shown below. All three kinematic patterns were very sensitive to the horizontal freestream, see Fig. 25 for the response to the 20\% freestream, and much less sensitive to the downward heading freestream. The downward freestream generally decreased lift, by suppressing vortex generation, while making the forward and backstrokes more symmetric as the vortical activity was washed away from the airfoil more quickly. Overall the general nature of the force history was kept intact. On the other hand the upward freestream had the opposite effect. Namely the vortex interactions were sustained for a longer period of time as the freestream held the wake closer to the airfoil and the increased angle of attack also served to accentuate the unsteady aerodynamics. This upward freestream may or may not have had a significant impact on the force history which was dependent on the kinematics. The horizontal freestream had the largest impact over the range of kinematic motions studied, sometimes more than doubling the lift felt for freestream strength of $20 \%$ of the maximum translational velocity, a relatively tame environmental contribution. 


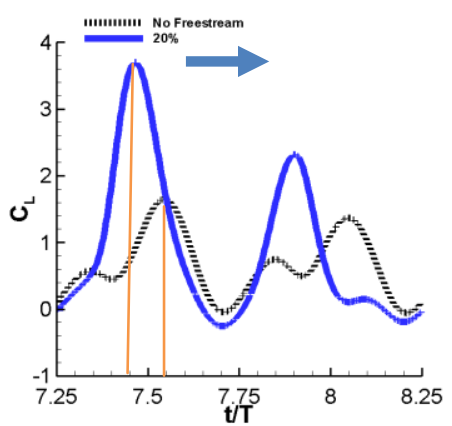

a) $2 h_{a} / c=3.0, \alpha_{a}=45^{\circ}$, and $\phi=90^{\circ}$

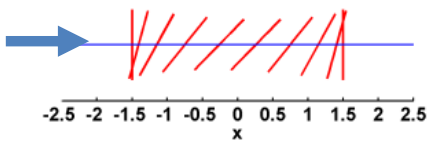

Figure 26. Force history (2D) and vorticity contours illustrating the vortex formation and interactions during the LEV dominated portion of the stroke at their respective maximal lift for b) a $20 \%$ strength headwind c) hover scenario with no freestream.

Figure 26 illustrates highlights the force history and flowfields at maximal lift for the kinematics expressed in Fig. 26a. Immediately apparent is the large impact on the instantaneous as well as time-averaged lift. To clarify, the lift coefficients are still normalized by the maximum translational velocity, i.e. the normalization is independent of the freestream. Flowfields are plotted during the headwind portion of the stroke (backstroke), and show that the headwind case exhibits a more developed and stronger LEV as well as stronger vorticity shedding from the trailing edge. The increased vortical activity created by the headwind, and then interacting with the airfoil in a favorable manner explains qualitatively the increase in performance during the backstroke. But why then the lift peaks during the forward stroke when in the presence of a tailwind?

The rather significant peak in lift is somewhat unexpected, but as will be seen occurs despite the tailwind and not because of it. This peak occurs after stroke reversal as the airfoil interacts with the previously shed wake, or wake-capture dominated portion of the stroke cycle. The hover case temporarily drops off in lift, see Fig. 27 a, whereas the $20 \%$ freestream case, now a tailwind, continues to increase in lift. Vorticity contours at their respective local maximums in lift, see Fig. 27b and Fig. 27c show a few striking differences, noticeably the strength and position of the previously shed vorticity. Due to the headwind during the backstroke, stronger vortices were created. Now on the return stroke, those vortices' strength, in addition to their position relative to the airfoil, significantly help promote vortex growth, see Fig. 27c. This interaction, resulting in a temporary enhancement, eventually plays itself out and a decline in lift ensues in what used to be dominated by the LEV, but now amounts to slower relative translational velocity. 


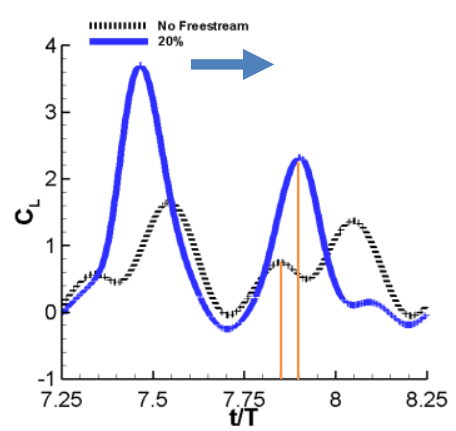

a) $2 h_{a} / c=3.0, \alpha_{a}=45^{\circ}$, and $\phi=90^{\circ}$

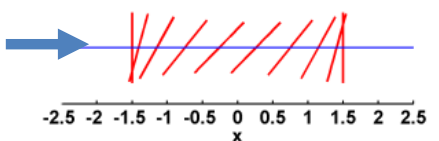

Figure 27. Force history (2D) and vorticity contours illustrating the vortex formation during the wakecapture dominated portion of the stroke at their respective local maximums in lift for b) hover c) $20 \%$ tailwind freestream.

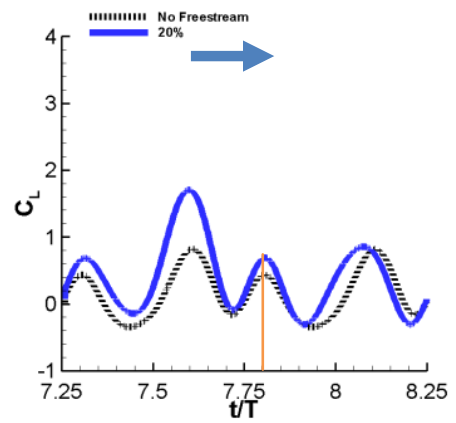

a) $2 h_{a} / c=3.0, \alpha_{a}=80^{\circ}$, and $\phi=90^{\circ}$

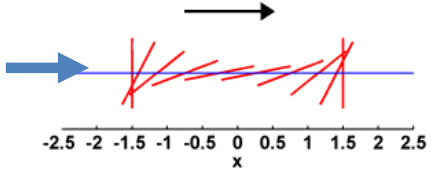

Figure 28. Force history (2D) and vorticity contours illustrating the vortex formation during the wakecapture dominated portion of the stroke at their respective local maximums in lift for b) hover c) $20 \%$ tailwind freestream.

To contrast the wake-capture impact just discussed, a case with a much smaller sensitivity during stroke reversal is illustrated, see Fig. 28. There is a small difference in the lift experienced, but looking at the corresponding flowfield vorticity contours for the hovering case, Fig. 28b, and the 20\% horizontal freestream, Fig. 28c, show a decent qualitative agreement. This case has kinematics which do not promote large vortical structures. As the actual wake influence 
is rather small, the forces felt for these kinematics upon stroke reversal are more dependent on the motions rather than influence from the previously shed wake.

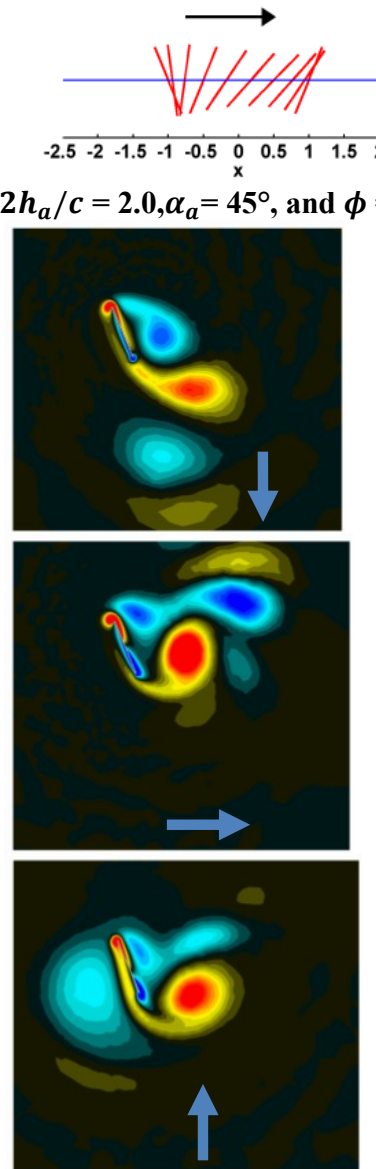

0.75
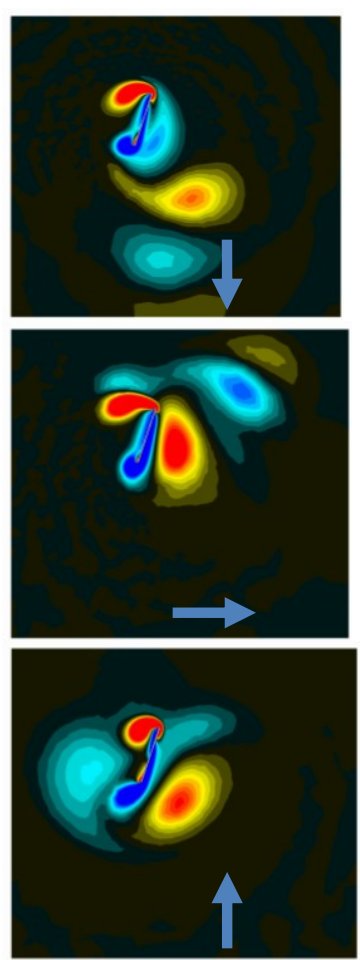

0.9

Stroke Starting

Figure 29. Vorticity contours of a delayed-rotation kinematic pattern and the flowfields associated with a $\mathbf{2 0 \%}$ freestream strength heading down/right/up.

Looking at the 2D force histories again, see Fig. 25, one will see that the response of a freestream not only depends on the kinematics but also its orientation. For some situations the qualitative nature of the flow doesn't change much over the course of the entire cycle nor are the forces too sensitive, see the vertical freestreams in Fig. 25a, or the downward freestream in Fig. 27c. On the other hand, the horizontal freestream has an appreciable impact for all of these kinematic patterns and specific points are mentioned above. The upward and downward freestreams don't necessarily elicit similar responses in opposite directions. This in turn brings into question the relevance of using effective angle of attack in these situations as the nature of the force history may respond more noticeably to the upward freestream than the downward freestream. See figure 29 where a $20 \%$ freestream imposed from different directions changes the flowfield and resulting force history, see figure $25 \mathrm{~b}$, significantly. 


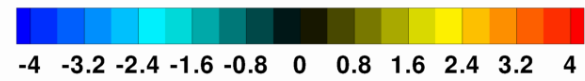

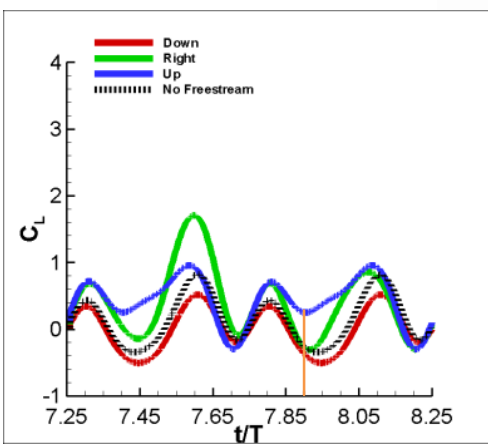

a) $2 h_{a} / c=3.0, \alpha_{a}=80^{\circ}$, and $\phi=90^{\circ}$

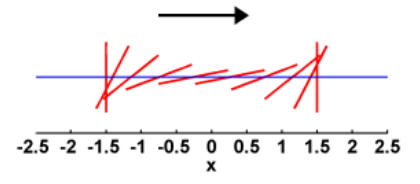

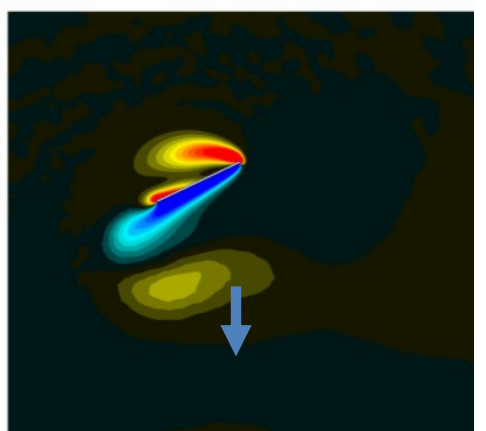

b) $20 \%$ downward freestream at $\mathrm{t} / \mathrm{T}=7.9$

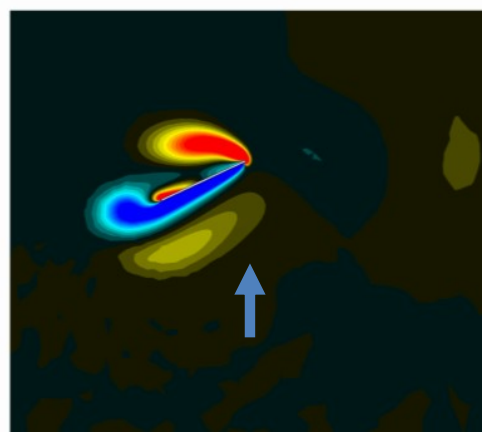

c) $20 \%$ upward freestream at $\mathrm{t} / \mathrm{T}=7.9$

Figure 30. Force history (2D) and vorticity contours illustrating the vortex formation between stroke reversal and their respective maximums in lift for b) $20 \%$ downward freestream c) $20 \%$ upward freestream.

For all of the synchronized rotation cases (which have positive angles of attack at all times), a $20 \%$ downward freestream does indeed decrease the lift and follows from an effective angle of attack type argument. On the other hand some cases have a much more pronounced sensitivity to the upward freestream. Figure 30 illustrates again the force histories for a $20 \%$ freestream at various orientations relative to the hover case, as well as the vorticity flowfield for the $20 \%$ upward and downward freestream cases at a time where the difference in force history between the two is pronounced. What is seen in Fig. 30b (20\% downward freesteam) and Fig. 30c (20\% upward freestream) is the increase in LEV and TEV formation as well as a more pronounced interaction with the wake as the upward freestream promotes the growth of the vortex structures and holds the wake in the vicinity for a longer period of time. The non-linear response in lift as the freestream lowers or raises the effective angle of attack is a product of these factors.

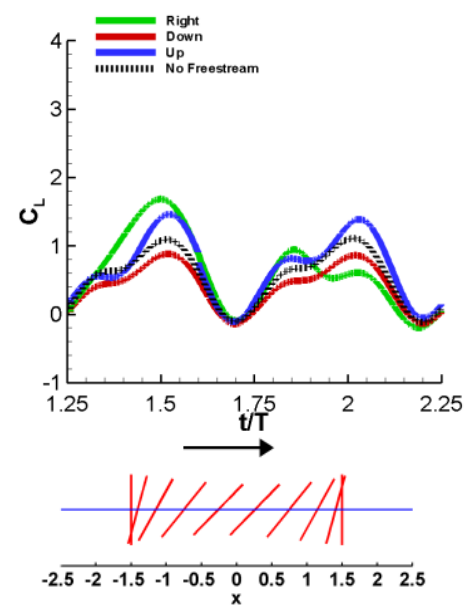

a) $2 h_{a} / c=3.0, \alpha_{a}=45^{\circ}$, and $\phi=90^{\circ}$

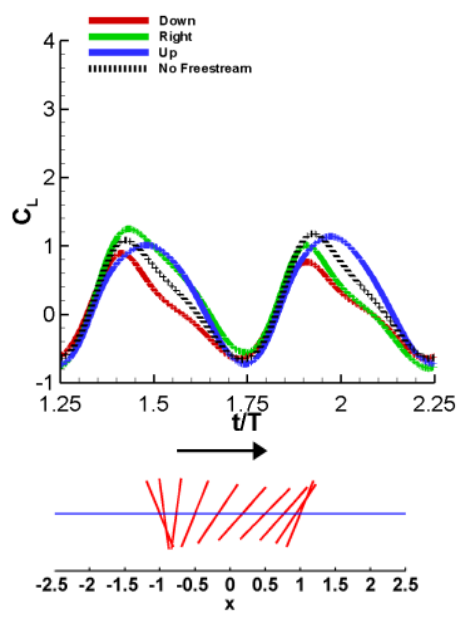

b) $2 h_{a} / c=2.0, \alpha_{a}=45^{\circ}$, and $\phi=60^{\circ}$

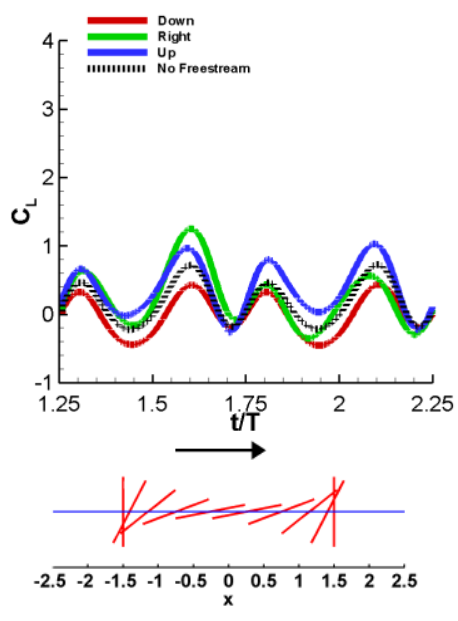

c) $2 h_{a} / c=3.0, \alpha_{a}=80^{\circ}$, and $\phi=90^{\circ}$

Figure 31. Three-dimensional $C_{L}$ in response to a freestream with a magnitude of $20 \%$ of the maximum plunging velocity heading in three distinct directions (down, right, and up) for three hovering kinematics. 


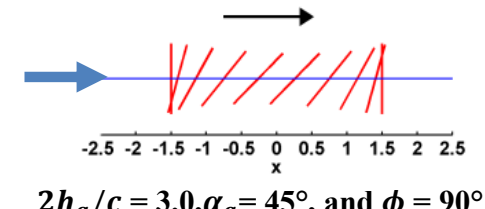

$2 h_{a} / c=3.0, \alpha_{a}=45^{\circ}$, and $\phi=90^{\circ}$
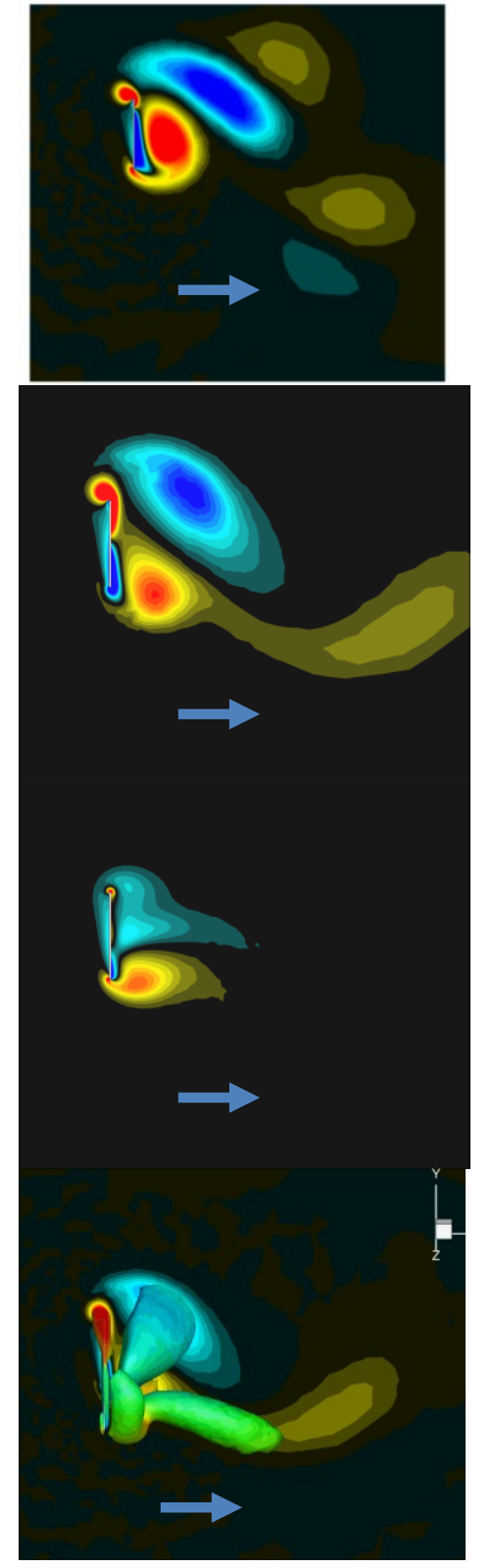

0.75

Stroke Starting

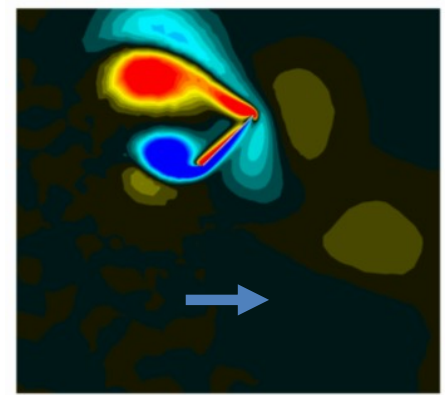

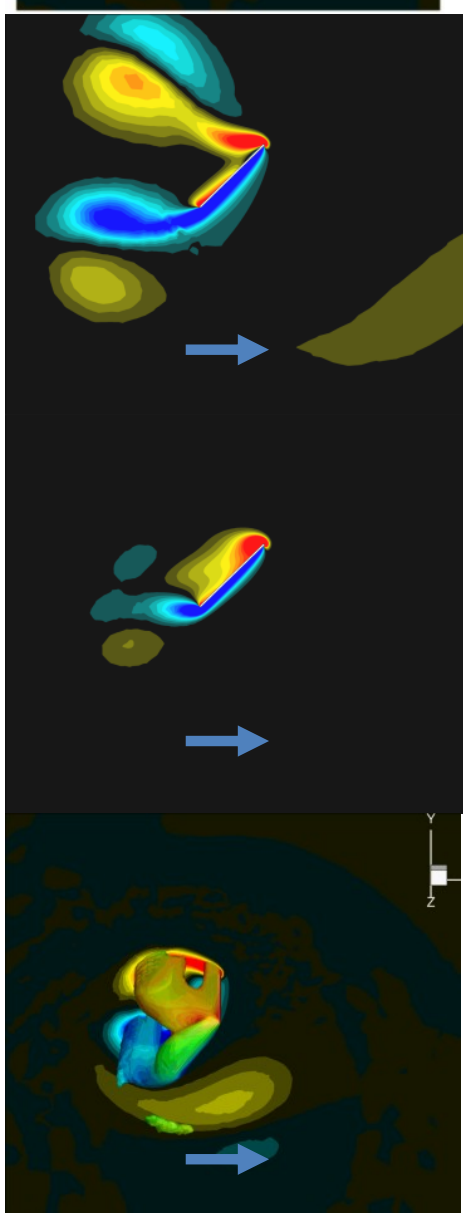

1.0

Mid-stroke

z-vorticity

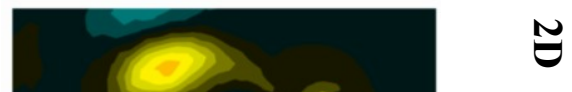

$\mathcal{V}$

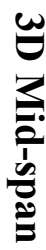

שِ

שَ

$\mathbf{t} / \mathbf{T}$

Stroke Ending

Figure 32. Vorticity contours during beginning, mid, and end-stroke for a 2D flatplate and at mid-span ( $\mathrm{z}=0$ ) and wingtip ( $\mathrm{z}=1.95)$ for a $3 \mathrm{D}$ flatplate with $\mathrm{AR}=4$ with a $20 \%$ freetream tailwind The $3 \mathrm{D}$ perspective shots show iso- $Q$ surfaces colored by $z$-vorticity. The blue arrow denotes freestream direction.

The 3D cases on the other hand were much less sensitive to the freestream, see Fig. 31. Note however the scale for the force histories was chosen such that they could be directly compared 
with the $2 \mathrm{D}$ cases and the freestream could be quite influential. The impact was non-negligible for a $20 \%$ strength freestream, but overall the nature of the flow was very similar for most cases. The downward freestream once again degraded lift, and the upward freestream enhanced it albeit to a lesser degree than in the $2 \mathrm{D}$ cases.

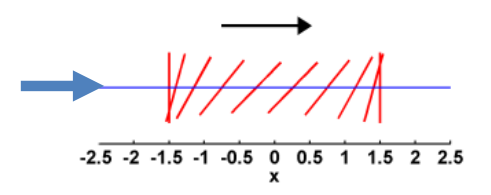

$2 h_{a} / c=3.0, \alpha_{a}=45^{\circ}$, and $\phi=90^{\circ}$

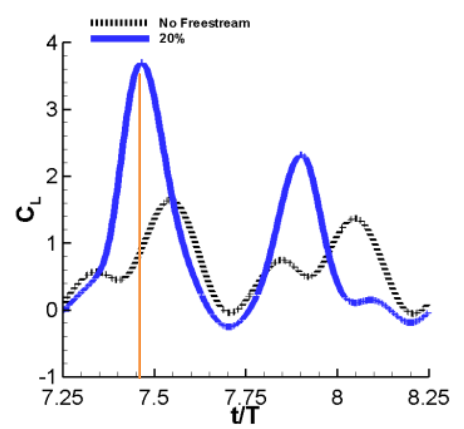

a) $2 \mathrm{D} \mathrm{C}_{\mathrm{L}}$

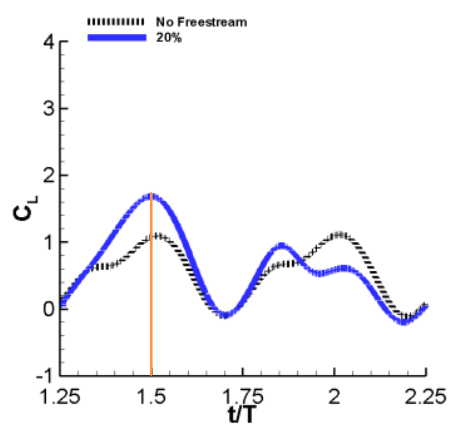

c) $3 \mathrm{D} \mathrm{C}$

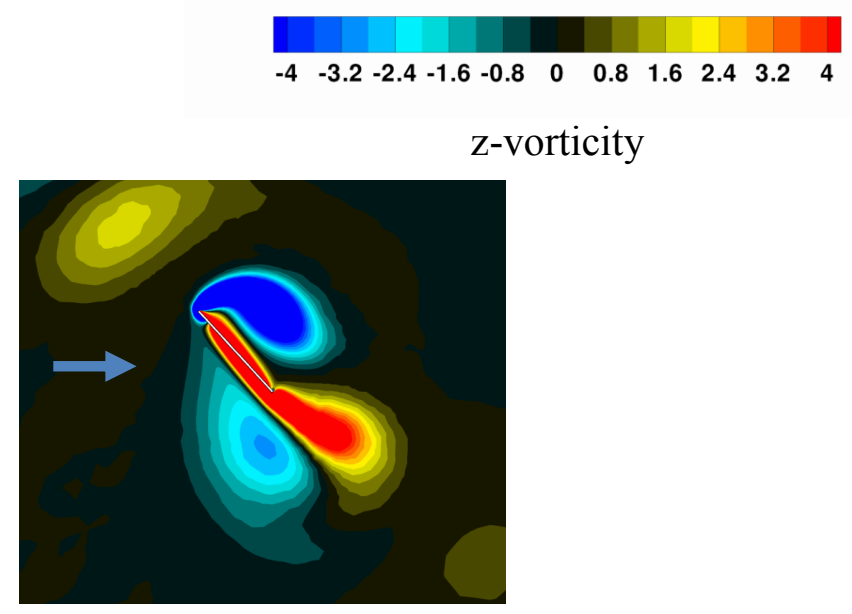

b) $2 \mathrm{D} \mathrm{t} / \mathrm{T}=7.45$

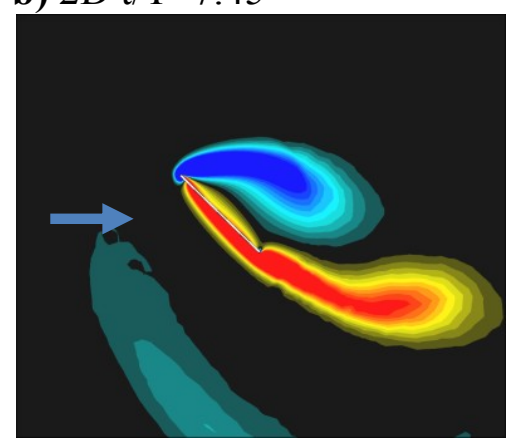

d) $3 \mathrm{D}$ Mid-span $(\mathrm{z}=0) \mathrm{t} / \mathrm{T}=1.5$

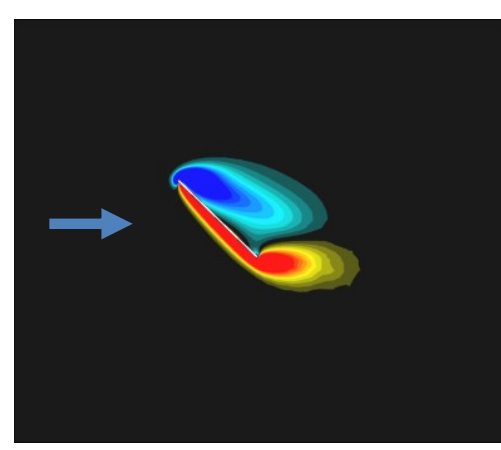

e) 3D Wing Tip $(\mathrm{z}=1.95)$

Figure 33. Force histories and vorticity contours at maximal lift with a $20 \%$ strength headwind for $2 \mathrm{D}$ and 3D $(\mathrm{AR}=4)$ flatplates.

Figure 32 highlights beginning, mid, and end stroke vorticity contours for a $20 \%$ freestream tailwind for 2D and at midspan and at the wing tips for 3D. The three-dimensional wing is unable to generate vortices of the same magnitude as the analogous $2 \mathrm{D}$ counterparts. This in turn directly impacts the wing's benefit from LEV interactions as well subsequent interactions with the previously shed wake. The spanwise variation of vorticity exhibited shows a decrease in LEV generation from mid-span to tip, and while the tip vortices, as evinced by the green colored iso- $\mathrm{Q}$ surface in Fig. 32 at mid-stroke, are prominent, they do not make up for the weakened LEV formation and wake interaction as experienced in 2D. Figure 33 illustrates the vorticity flowfields during maximal lift, during the headwind, resulting in a 2D lift almost twice as large. 


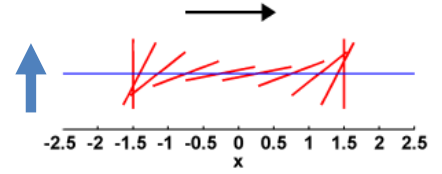

$2 h_{a} / c=3.0, \alpha_{a}=80^{\circ}$, and $\phi=90^{\circ}$
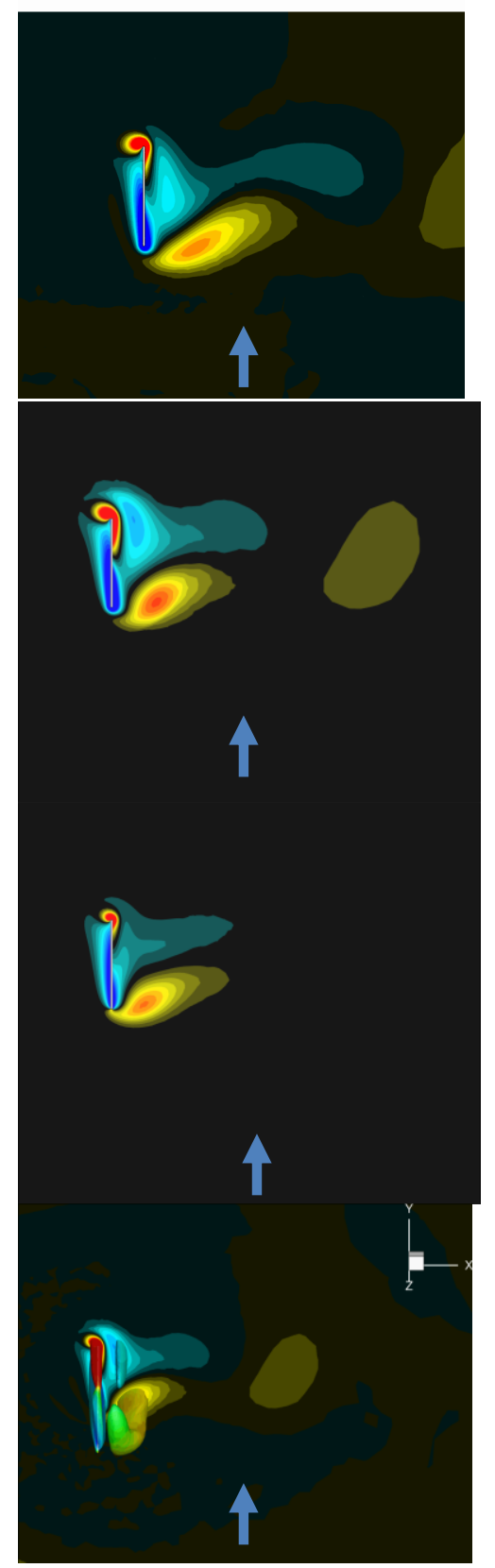

0.75

Stroke Starting

Figure 34. 2D/3D vorticity contours in the presence of an upward freestream for kinematics with synchronized rotation and low angles of attack. The 3D perspective shows an iso- $Q$ surface colored by $z-$ vorticity from midspan to the wing tip.
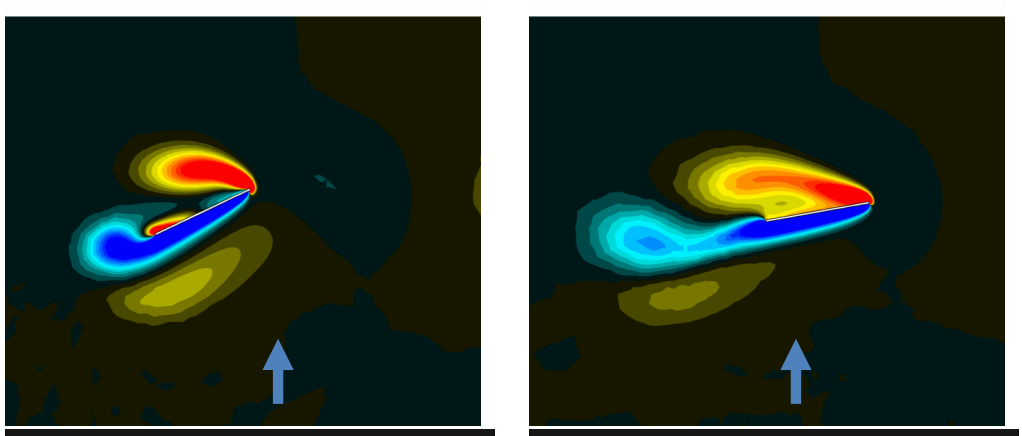

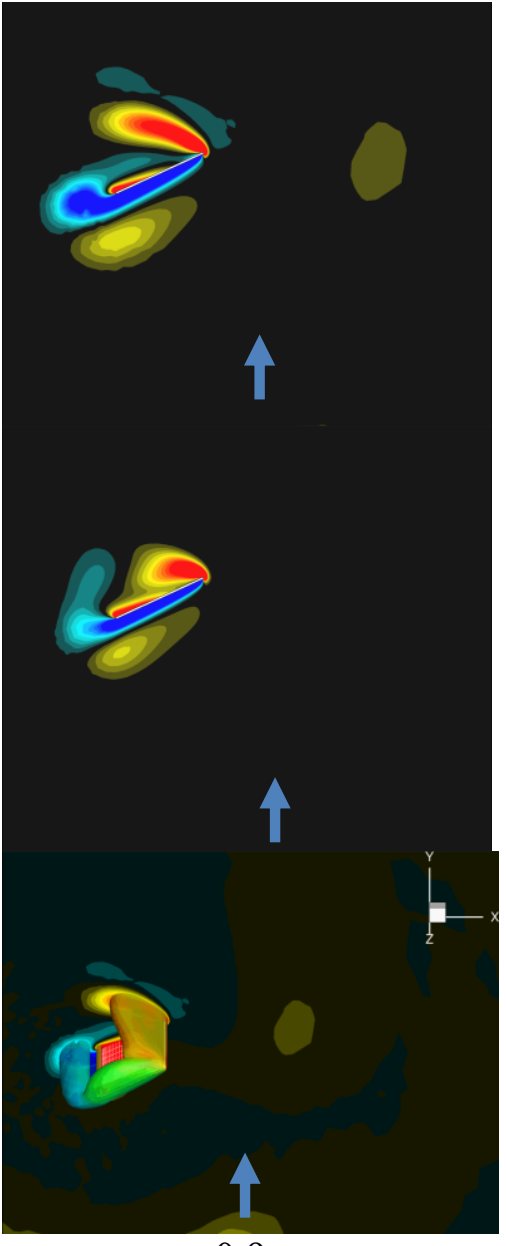

0.9

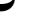


This discrepancy in sensitivity to freestream between 2D and 3D shows up across the range of kinematic motions. A limited subset of kinematic motions showed very similar force histories in the time averaged sense as well as instantaneously, see section 3.6, when not under the influence of an external freestream. Kinematics in this region of the design space shared low angles of attack across much of the flapping cycle and synchronized rotation, limiting the high angular velocities and angles of attack to the end of the stroke where translational velocity was minimized. This in turn tended to limit vortex size, strength, formation, and influence. As the freestream is introduced, see figure $30 \mathrm{a}$ and figure $31 \mathrm{c}$ for $2 \mathrm{D}$ and $3 \mathrm{D}$ force histories in the presence of a $20 \%$ freestream, the response is not uniform across the span of the finite wing. The downward freestream (20\% strength) tends to suppress the vortex dynamics and as such the $2 \mathrm{D}$ and 3D force histories remain quite similar. The horizontal freestream, most notably during the headwind, and the upward freestream, see figure 34, on the other hand show differences due to the $3 \mathrm{D}$ nature of the flow. In the absence of a prominent freestream we saw that the $3 \mathrm{D}$ effects could accentuate the $2 \mathrm{D}$ lift by creating a low pressure zone at the tip and by anchoring an LEV that would otherwise detach earlier from the wing. An interesting question, only partially addressed in the current work due to simulation resources, is to what extent the interplay between kinematic motions and the freestream strength and orientation can be manipulated.

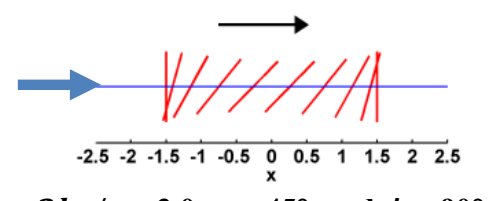

$2 h_{a} / c=3.0, \alpha_{a}=45^{\circ}$, and $\phi=90^{\circ}$
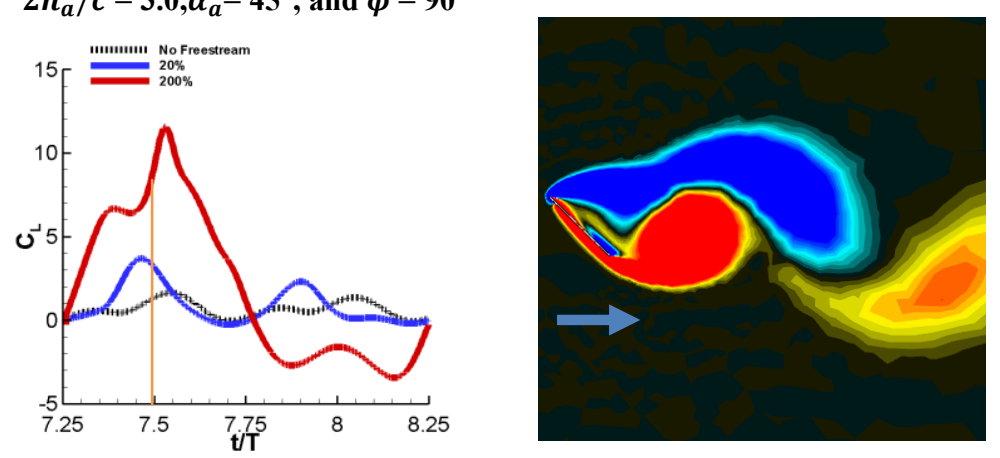

a) $2 \mathrm{D} \mathrm{C}$

b) $2 \mathrm{D} t / \mathrm{T}=7.5$

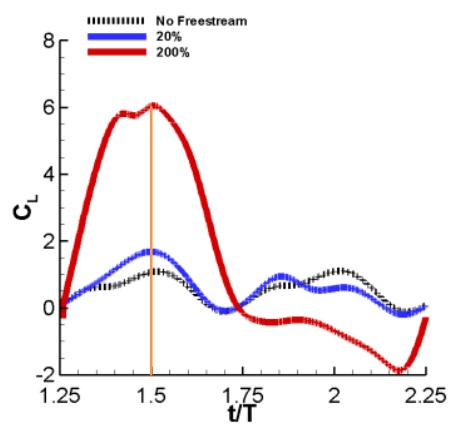

c) $3 \mathrm{D} \mathrm{C}$

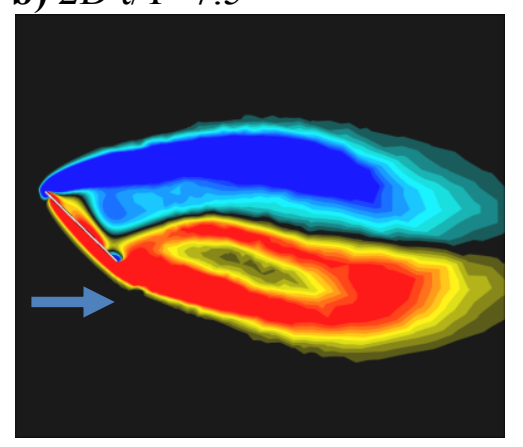

d) $3 \mathrm{D}$ Mid-span $(\mathrm{z}=0) \mathrm{t} / \mathrm{T}=1.5$

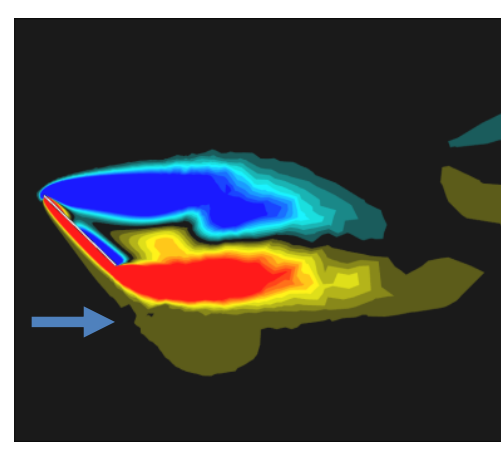

e) $3 \mathrm{D}$ Wing Tip $(\mathrm{z}=1.95)$

Figure 35. 2D (a) and 3D (c) lift time histories of lift in the presence of a 200\% strength horizontal freestream compared to its $20 \%$ and no freestream counterparts. Vorticity contours 2D (b), 3D midspan (d), and 3D wingtip (e) during maximum relative velocity and highest angle of attack. 
All of the results in this section thus far examine a freestream strength whose magnitude is a fraction of the reference velocity. Select cases were chosen where the freestream strength was increased to $200 \%$ the reference velocity strength and whose direction was from left to right. As the forces were normalized by the maximum translational velocity, i.e. independent of the freestream, the impact of a strong freestream was quite large, see figures $35 \mathrm{a}$ and $35 \mathrm{c}$. Note that the scales have changed between these two to properly show the force histories. The vorticity contours have been plotted during the maximum relative velocity and highest angle of attack, and show significant vortical activity. It is worth noting, that because the vortex activity is washed away from the wing fast enough, the force histories in both $2 \mathrm{D}$ and $3 \mathrm{D}$ are periodic in nature and not chaotic. It is also seen that during the whole stroke experiencing a tailwind, due to the freestream being twice as strong as the maximum translational velocity, the wing is essentially flying upside down and thus experiencing negative lift. In contrast to the weaker freestreams, the interaction with the previously shed vortices cannot compensate for the effective decrease in relative translational velocity.
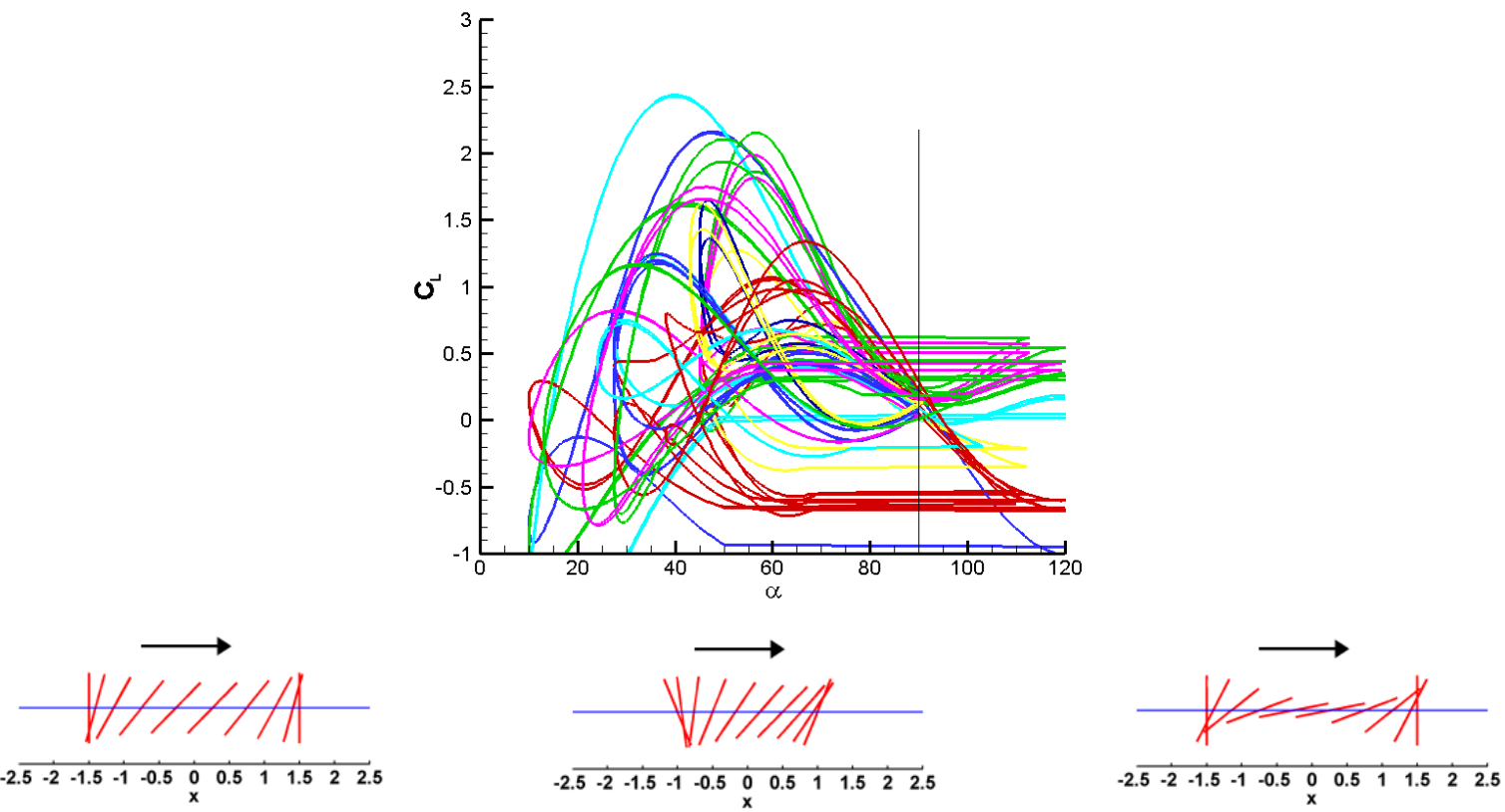

Figure 36. Illustration cautioning the use of effective angle of attack as measure of lift in the hovering context.

The lift coefficients of the three kinematic patterns studied in this section have been plotted versus the effective angle of attack for freestream strengths of $5 \%, 10 \%$, and $20 \%$ with orientations heading down, right, and up.

To conclude this section, a caution on the use of effective angle of attack, and therefore quasisteady theories, in the context of hovering flapping wing flight will be illustrated. This is not meant to imply quasi-steady theories are not useful, but rather that the applicability of such a model can be limited and that the context and assumptions of the model should be revisited before applying them in an arbitrary hovering context, i.e. deviating from the kinematic motions expressed in the training of the quasi-steady model can invalidate its usefulness in a seemingly related case. Figure 36 illustrates the kinematic patterns highlighted in this section under the influence of freestream strengths of $5 \%, 10 \%$, and $20 \%$ in the down, right, and upward directions. The take away message is that within a certain range, an arbitrary value of lift coefficient can be attained for any effective angle of attack. Figure 36 shows, for example that at 
an effective angle of attack of $45^{\circ}$ can yield lift coefficients from approximately -1 to +2.5 . The transient and time history effects are not negligible.

\section{Summary and Conclusion}

In this study we examined hovering kinematics of a two-dimensional and three-dimensional flat plate $(A R=4)$ with the aid of surrogate models at a Reynolds number of 100 . Regions for which large discrepancies (see figure 6) between the time averaged forces in 2D versus 3D were identified with the surrogate models and the flowfield solutions from CFD were reexamined with dominate flow features highlighted for each grouping of kinematics. Regarding the previous literature the jet experimentally seen in the studies of Freymuth ${ }^{32}$ was seen to have a noticeable effect on the instantaneous force histories when enough care with spatial and temporal resolutions was taken. In the study of Sane and Dickinson ${ }^{27}$, consistent conclusions are reached for obtaining optimal performance, e.g., keep fairly high angles of attack for as much of the stroke as possible. There are differences though in the sensitivity to plunging amplitude, which was small in the current study, but was relevant in the flapping about a joint method employed in their study. Furthermore, it would appear that the wake-capture portion of the cycle played a larger role as evidenced by the force peaks they saw. In the current study the LEV peak seems to influence the aerodynamic forces much more so than the wake-capture peak. Open questions regarding Reynolds number effects, flapping kinematics, and vortex dynamics are planned for future study. Furthermore, new insight into the fluid physics is gained, including the effects of tip vortices.

\section{Hover:}

Regarding the fluid physics and aerodynamic implications during hover, we observed:

(i) The role of the leading edge vortex and associated delayed stall plays a dominant role in the determination of lift in both two- and three-dimensional cases. During delayed rotation the starting vortices detached causing a weaker LEV during mid-stroke. In three-dimensional case, the tip vortices during delayed rotation tended to repel the starting vortices at the leading and trailing edges, thus leading to a substantial variation in the spanwise flow. However, during advanced rotation, the starting vortex remained attached with no distinction between the starting vortex and the LEV, and enhanced lift. Angle of attack, angular velocity, translational velocity, and translational acceleration all impact the formation and evolution of the LEV.

(ii) The manifestation of the wake-capture mechanism in three-dimensional case has been seen to change as the behavior of the shed vortices may differ between two- and threedimensional cases. This is illustrated by one case where the shed two-dimensional vortices would remain in the plane of the plunging motion. The three-dimensional counterparts are shed at angles such that they leave plane of the plunging wing and do not collide with the wing upon its return.

(iii) In two-dimensional cases with higher angles of attack, i.e., lower pitching amplitudes, a persistent downward jet like flow feature was seen to form in the wake sustained by the influence of shed vortices. The jet was absent in the two-dimensional cases with lowest angles of attack as there was also no large scale vortex shedding. In the low aspect ratio three-dimensional case at $R e=100$, the presence and influence of an induced jet is not as clear cut. There are pockets of momentum encountered on return strokes, but there is no coherent reverse Karman vortex street reinforcing the jet due to the more complex three-dimensional vortex interactions. 
(iv) There was significant variance in the spanwise distribution of forces in the threedimensional cases. Cases which suppressed the unsteady mechanisms, those with the highest angular amplitudes and thus low angles of attack, and small velocity when vertical (e.g. near synchronized hovering) appeared to have a relatively constant response along the span. In contrast three-dimensional cases with prominent tip vortices exhibited significant variations along the span which did not have a strong correlation to the two-dimensional lift values experienced.

(v) At the present Reynolds number the three-dimensional flow physics and tip effects can augment the lift by: a) the presence of a low pressure region near the tip, b) the anchoring of an otherwise shed vortex (in 2D) near the tip, and c) weakening the pocket of downward momentum encountered on a return stroke. A competing effect is the induced downwash of tip vortex and the reduced effective angle of attack along the span serving to reduce lift.

\section{Environmental Sensitivity:}

(i) As flapping wing frequencies are typically much faster than that of environmental perturbations, gusts can be modeled as a constant freestream for many practical applications.

(ii) The sensitivity to the gust for various kinematic parameters was largely determined on how much influence it had on the vortex dynamics. Headwinds with a modest $20 \%$ strength could significantly enhance the LEV generation and this in turn provided stronger wake interactions on the return stroke. For most kinematic motions, within the ranges studied, a 20\% horizontal freestream increased the $2 \mathrm{D}$ lift despite half of the stroke experiencing a tailwind.

(iii) The 3D cases, while still affected appreciably, were noticeably less sensitive to the gusts than their 2D counterparts due to the weakened vortex dynamics generally occurring in $3 \mathrm{D}$.

(iv) The impact of a gust was tied to its strength, orientation, and what kinematics it encountered. Those affected by the gust saw the manifestation through its role in LEV, tip vortex, and wake generation and interaction. Those kinematics with little of the aforementioned vortex dynamics saw the smallest sensitivity to the gust.

(v) By changing the effective angle of attack on select kinematic motions with various freestream orientations and strengths, different values at the same effective angle of attacks were obtained. Hence a cautionary note on the application of quasi-steady models in the hovering context is warranted.

\section{Surrogate Modelling:}

Often as case studies in the literature are performed, or independent variables varied in a parametric study, it can become difficult to place the findings in the proper context thus making it difficult to glean the relevant information and compare with findings of other researchers. The surrogate process more readily illuminates the global perspective of variables' impact as well as the limitations of the findings, making identification of the applicability much more apparent. The surrogates modeling techniques provided a useful method for approximating otherwise computationally expensive simulations without sacrificing fidelity. Meanwhile they proved an efficient method for analyzing trends seen throughout the design space and possible points of interest. Coupled with instantaneous force histories and flow field measurements they can provide insight to the complex interplay of the physical mechanisms involved.The surrogate modeling techniques further revealed that: 
(i) The weighted average surrogate agreed very well at the independent test points. While the number of independent test points was limited, the error was often less than a few percent. The maximum error that was found was less than $15 \%$. The agreement shows promise for using surrogates, namely weighted average surrogates, as a reduced order model for complex engineering design in the MAV context.

(ii) The hierarchy of variable sensitivity in the time averaged lift changed between twodimensional and three-dimensional hover. In two-dimensions the importance was 1) angular amplitude 2) phase lag and 3) plunging amplitude. In three-dimensions the hierarchy switches to 1) phase lag 2) angular amplitude and 3) plunging amplitude. This is in large part from the tip vortices in three-dimensional simulations not suffering as large a penalty from the lift valley that would occur between wake-capture and delayed stall. This difference in two-dimensions and three-dimensions is partially attributed to the reduced influence of the jet interaction in three-dimensional simulations.

(iii) Interestingly, the power required remained largely the same between two-dimensional and three-dimensional design spaces. This implies that the unsteady effects which influence the drag can in some contexts be reduced down to their two-dimensional counterparts. Note the pressure force, and not the viscous force, was still the dominant component of the drag felt.

(iv) Regions for which 2D kinematics outperformed 3D in hover and vice-versa were identified. Furthermore the density of points near the Pareto front was higher in three dimensions implying more design variable combinations that are close to optimal in terms of power and lift trade-offs.

In summary, for steady state flow around a wing, the energy used to create tip vortices is not deposited in the flow in a fashion which efficiently contributes to the lift. The finding that a three-dimensional low aspect ratio wing can produce higher lift than a two-dimensional airfoil is not supported by the classical stationary wing theory, which suggests that wing tip vortices decrease performance. In unsteady flow around a hovering wing, however, the tip vortices need not be a drag on the wing.

\section{Acknowledgments}

The work supported here has been supported in part by the Air Force Office of Scientific Research's Multidisciplinary University Research Initiative (MURI) grant and by the Michigan/AFRL (Air Force Research Laboratory)/Boeing Collaborative Center in Aeronautical Sciences. F. Viana, and T. Goel are gratefully acknowledged for their useful suggestions and insight during discussions. 
Appendix A 2D/3D Lift Coefficient Histories for Selected Cases

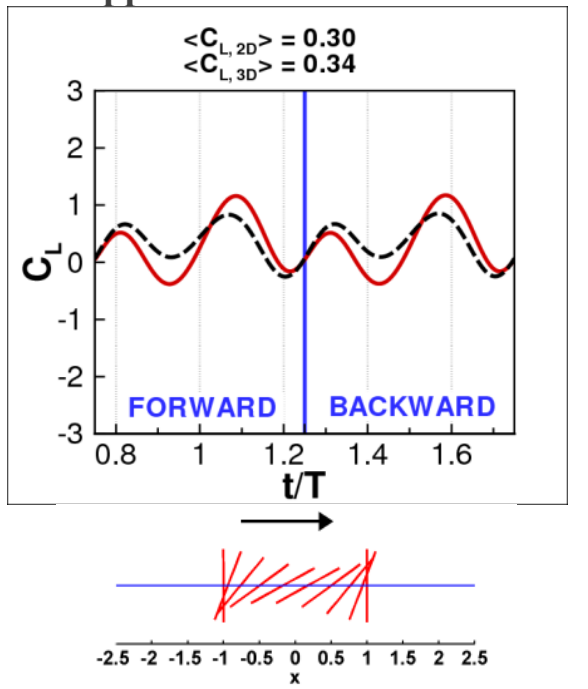

(a) $2 h_{a} / c: 2.0, \alpha_{a}=62.5^{\circ}$ and $\phi=90^{\circ}$

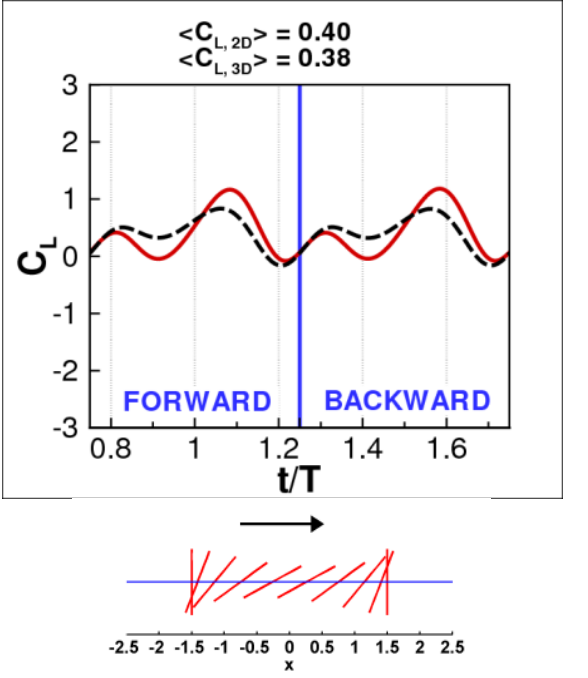

(b) $2 h_{a} / c: 3.0, \alpha_{a}=62.5^{\circ}$ and $\phi=90^{\circ}$

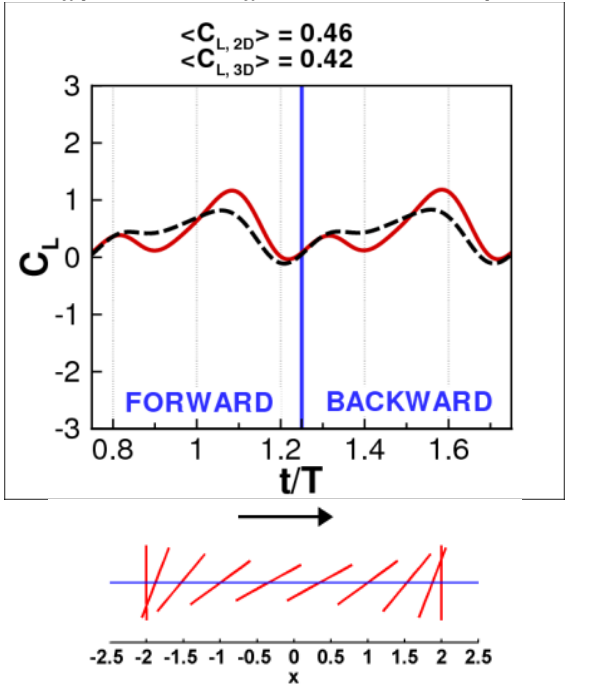

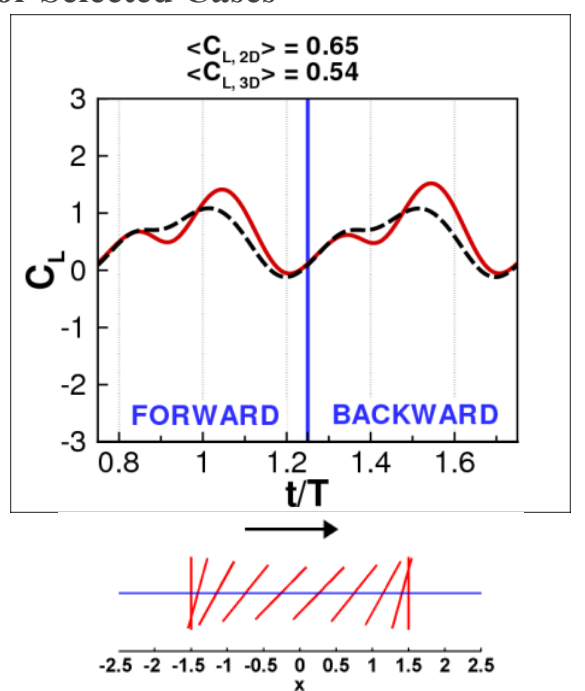

(d) $2 h_{a} / c=3.0, \alpha_{a}: 45^{\circ}$ and $\phi=90^{\circ}$

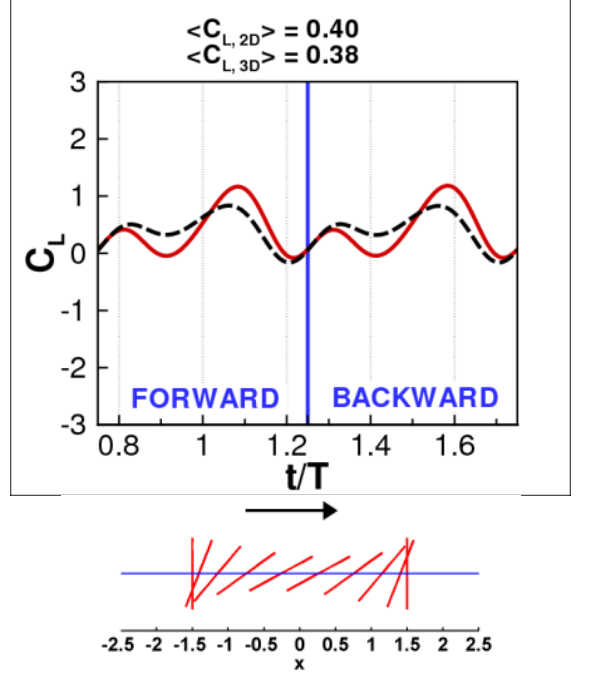

(e) $2 h_{a} / c=3.0, \alpha_{a}: 62.5^{\circ}$ and $\phi=90^{\circ}$

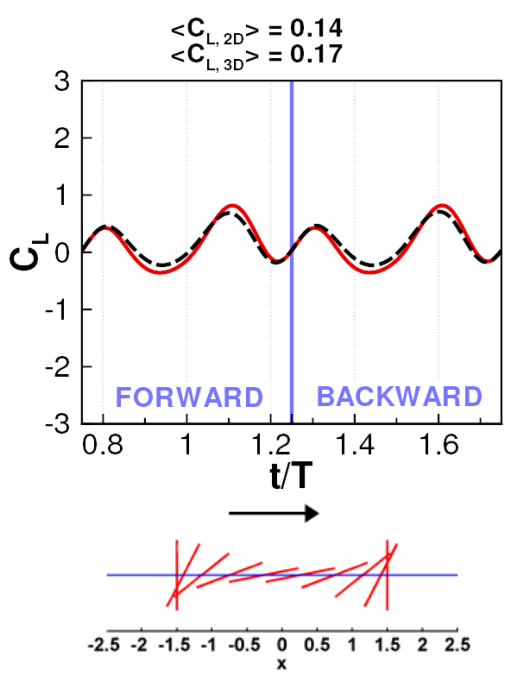


(c) $2 h_{a} / c: 4.0, \alpha_{a}=62.5^{\circ}$ and $\phi=90^{\circ}$

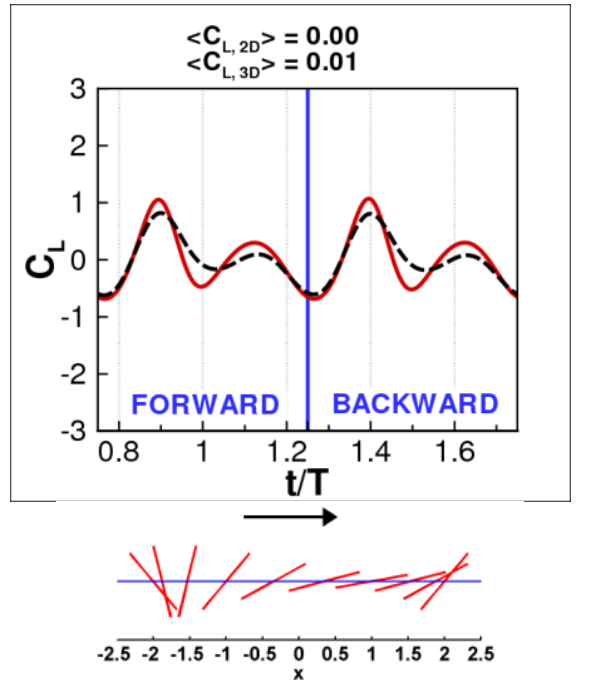

(g) $2 h_{a} / c=4.0, \alpha_{a}=80^{\circ}$ and $\phi=60^{\circ}$

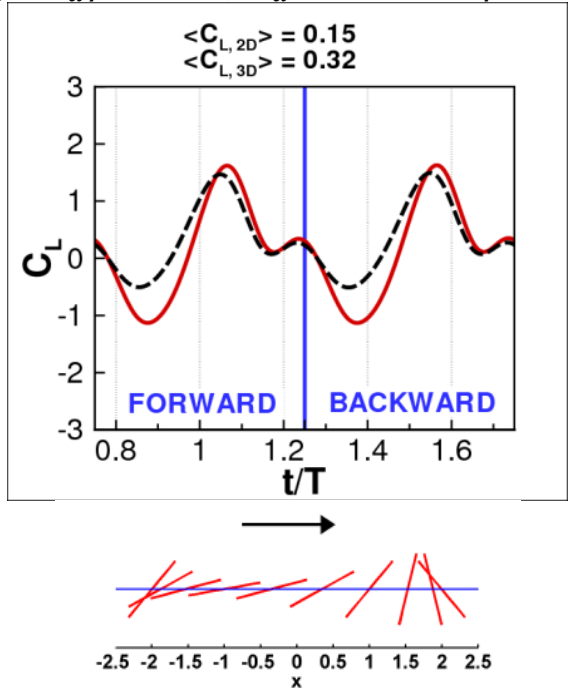

(h) $2 h_{a} / c=4.0, \alpha_{a}=80^{\circ}$ and $\phi=120^{\circ}$ (f) $2 h_{a} / c=3.0, \alpha_{a}: 80^{\circ}$ and $\phi=90^{\circ}$

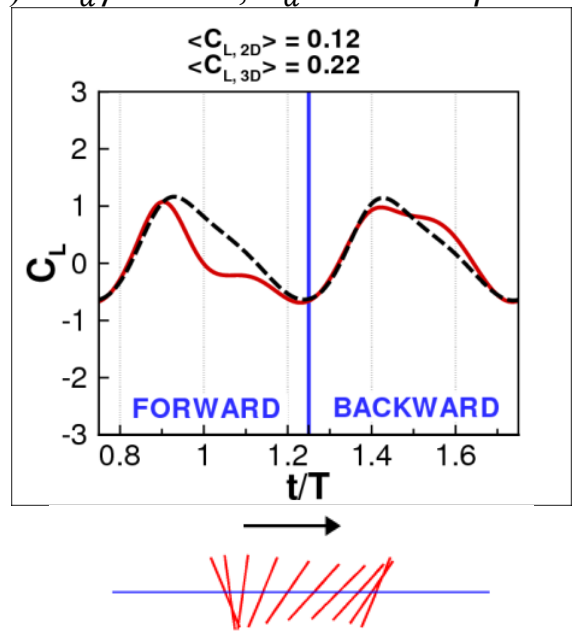

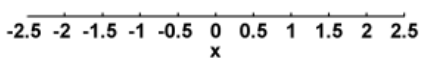

(i) $2 h_{a} / c=2.0, \alpha_{a}=45^{\circ}$ and $\phi=60^{\circ}$

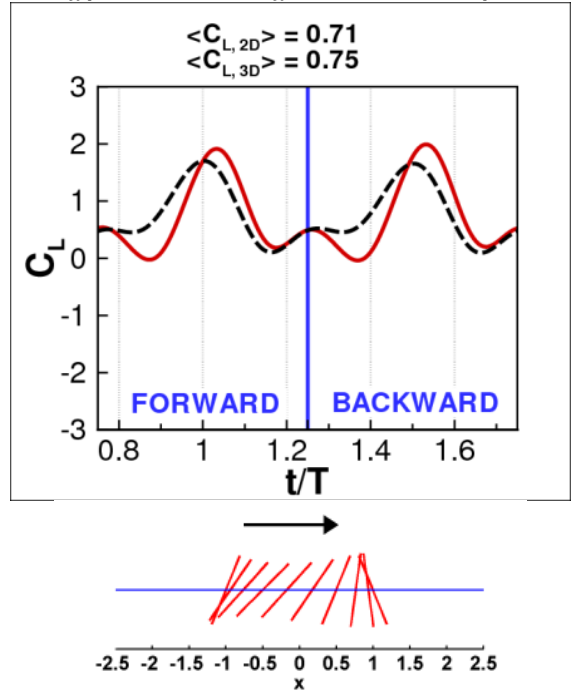

(j) $2 h_{a} / c=2.0, \alpha_{a}=45^{\circ}$ and $\phi=120^{\circ}$

FIGURE 37. Time histories of the lift coefficients for selected cases

(a-c) as $2 h_{a} / c$ is increased from 2.0 to 4.0 holding $\alpha_{a}=62.5^{\circ}$ and $\phi=90^{\circ}$ (d-f) as $\alpha_{a}$ is increased from $45^{\circ}$ to $80^{\circ}$ while holding $2 h_{a} / c=3.0$ and $\phi=90^{\circ}\left(\mathrm{g}\right.$,h) examining delayed rotation $\phi=60^{\circ}$ and advanced rotation $\phi=120^{\circ}$ while holding $2 h_{a} / c=4.0 \alpha_{a}=80^{\circ}(\mathrm{i}, \mathrm{j})$ examining delayed rotation $\phi=60^{\circ}$ and advanced rotation $\phi=$ $120^{\circ}$ while holding $2 h_{a} / c=2.0 \alpha_{a}=4^{\circ}$. [red,solid: 2D, black,dashed: 3D] 
Appendix B 2D/3D Drag Coefficient Histories for Selected Cases

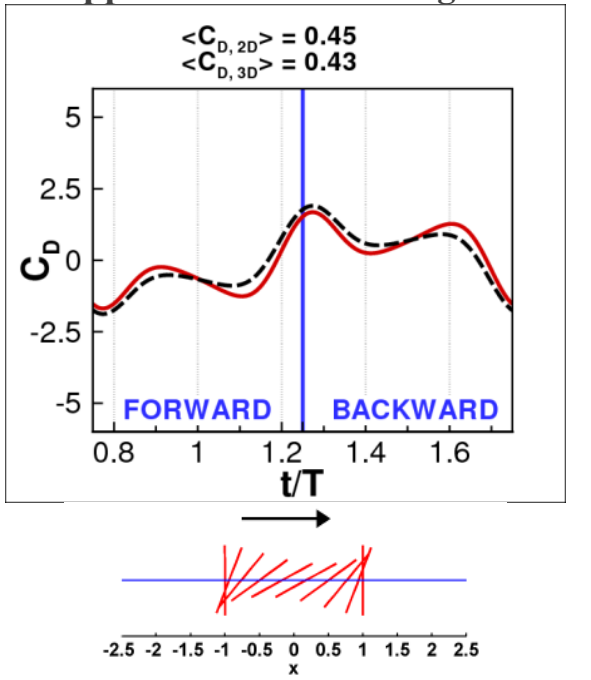

(a) $2 h_{a} / c: 2.0, \alpha_{a}=62.5^{\circ}$ and $\phi=90^{\circ}$

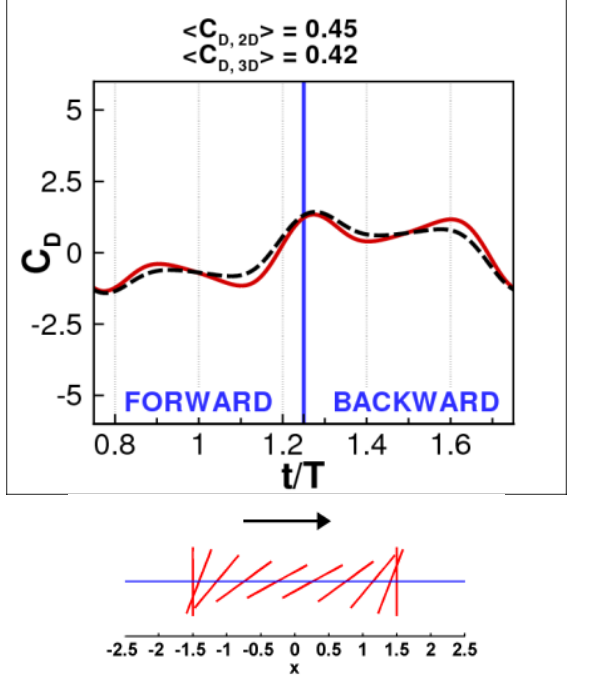

(b) $2 h_{a} / c: 3.0, \alpha_{a}=62.5^{\circ}$ and $\phi=90^{\circ}$

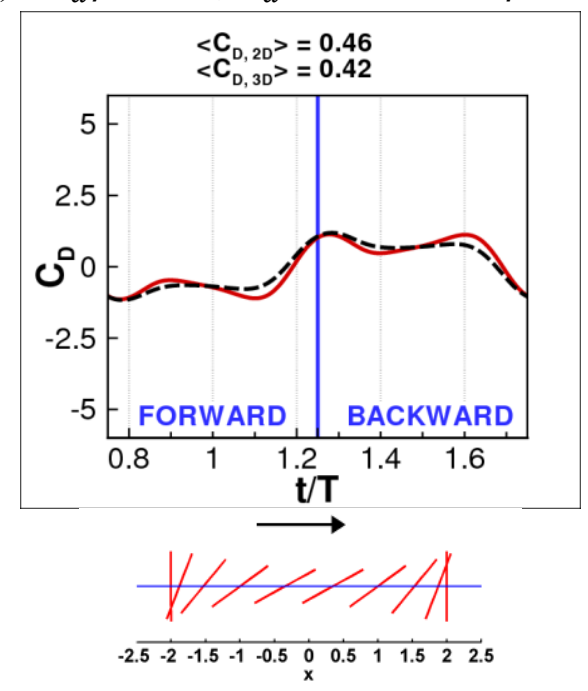

(d) $2 h_{a} / c=3.0, \alpha_{a}: 45^{\circ}$ and $\phi=90^{\circ}$

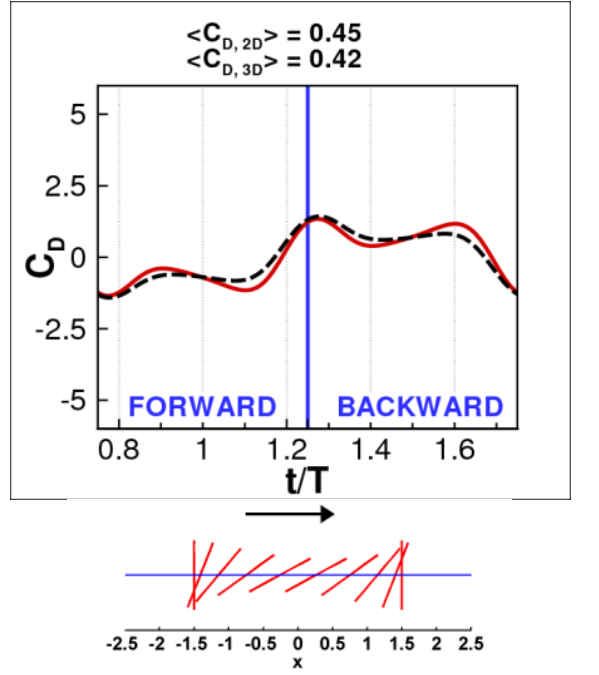

(e) $2 h_{a} / c=3.0, \alpha_{a}: 62.5^{\circ}$ and $\phi=90^{\circ}$

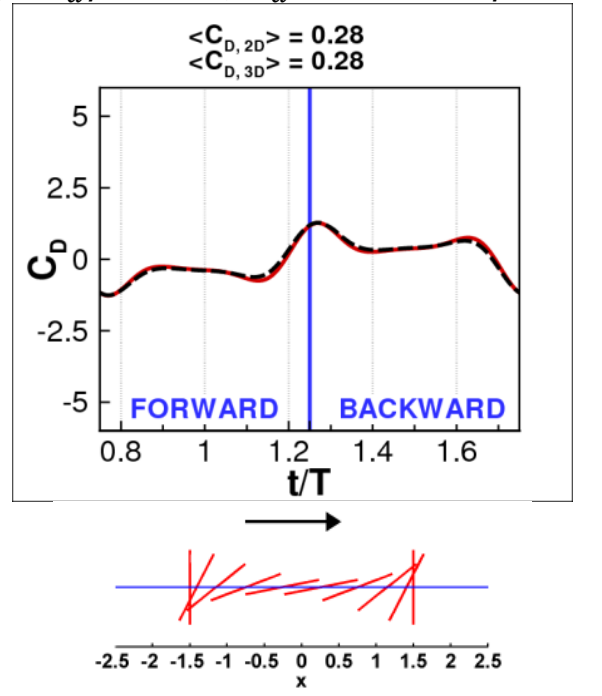


(c) $2 h_{a} / c: 4.0, \alpha_{a}=62.5^{\circ}$ and $\phi=90^{\circ}$

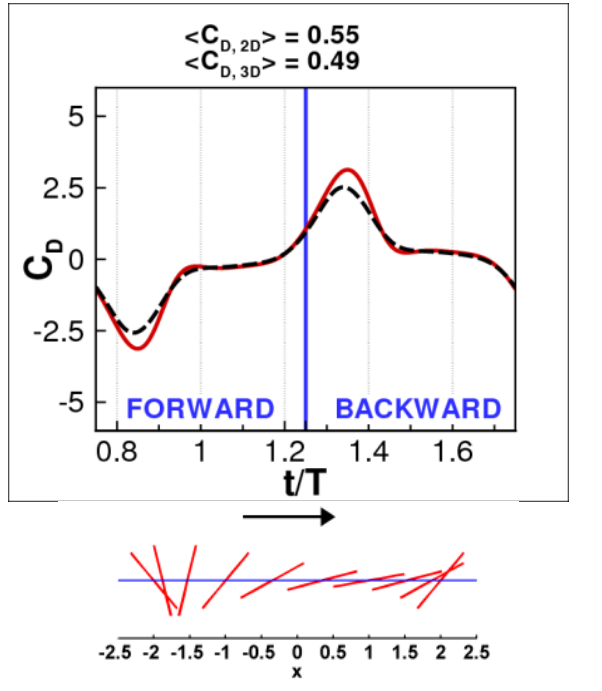

(g) $2 h_{a} / c=4.0, \alpha_{a}=80^{\circ}$ and $\phi=60^{\circ}$

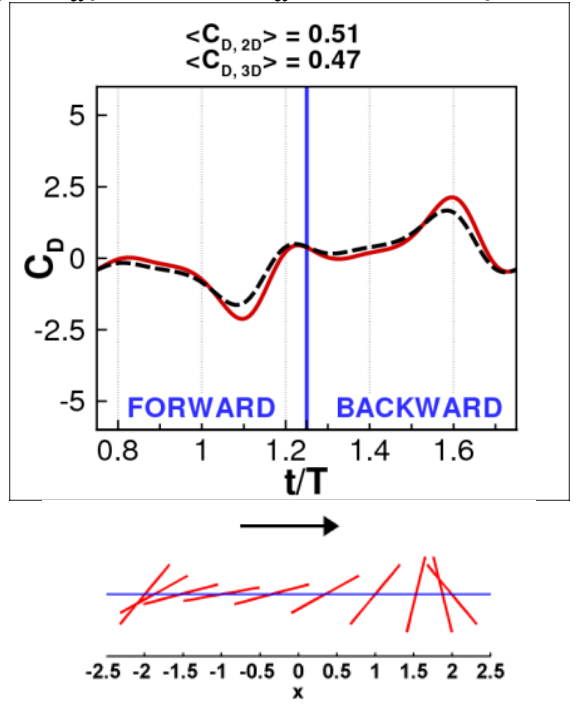

(h) $2 h_{a} / c=4.0, \alpha_{a}=80^{\circ}$ and $\phi=120^{\circ}$ (f) $2 h_{a} / c=3.0, \alpha_{a}: 80^{\circ}$ and $\phi=90^{\circ}$

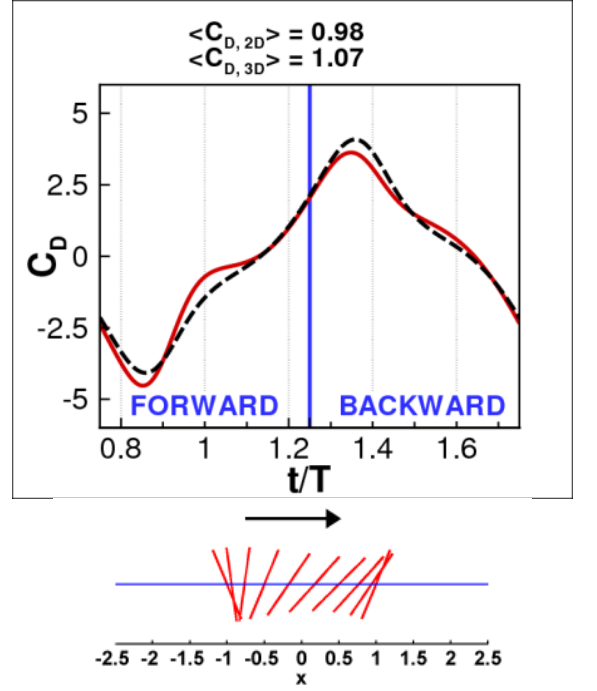

(i) $2 h_{a} / c=2.0, \alpha_{a}=45^{\circ}$ and $\phi=60^{\circ}$

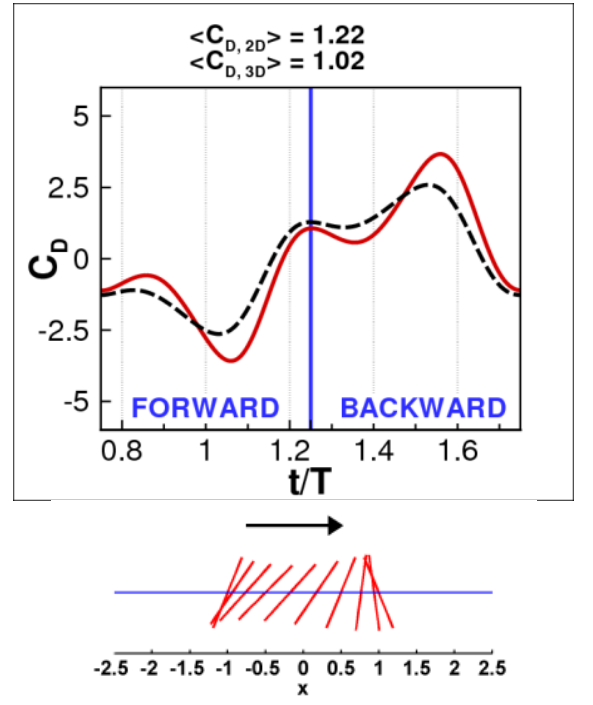

(j) $2 h_{a} / c=2.0, \alpha_{a}=45^{\circ}$ and $\phi=120^{\circ}$

FIGURE 38. Time histories of the drag coefficients for selected cases (a-c) as $2 h_{a} / c$ is increased from 2.0 to 4.0 holding $\alpha_{a}=62.5^{\circ}$ and $\phi=90^{\circ}$ (d-f) as $\alpha_{a}$ is increased from $45^{\circ}$ to $80^{\circ}$ while holding $2 h_{a} / c=3.0$ and $\phi=90^{\circ}(\mathrm{g}, \mathrm{h})$ examining delayed rotation $\phi=60^{\circ}$ and advanced rotation $\phi=120^{\circ}$ while holding $2 h_{a} / c=4.0 \alpha_{a}=80^{\circ}(\mathrm{i}, \mathrm{j})$ examining delayed rotation $\phi=60^{\circ}$ and advanced rotation $\phi=$ $120^{\circ}$ while holding $2 h_{a} / c=2.0 \alpha_{a}=45^{\circ}$. [red,solid: 2D, black,dashed: 3D]

\section{References}

${ }^{1}$ W. Shyy, Y. Lian, J. Tang, D. Viieru, and H. Liu, Aerodynamics of Low Reynolds Number Flyers. (Cambridge University Press, New York, 2008).

${ }^{2}$ T.J. Mueller and J.D. DeLaurier, “Aerodynamics of Small Vehicles,” Annual Review of Fluid Mechanics 35, 89-111 (2003).

${ }^{3}$ F. Bohorquez, P. Samuel, J. Sirohi, L. Rudd, and D. Pines, "Design Analysis and Hover Performance of a Rotary Wing Micro Air Vehicle," Journal of American Helicopter Society 48, No. 2, 80-90 (2003).

${ }^{4}$ W. Shyy, M. Berg, and D. Ljungqvist, "Flapping and Flexible Wings for Biological and Micro Air Vehicles," Progress in Aerospace Sciences 35, 455-505 (1999). 
${ }^{5}$ R.S. Fearing, K.H. Chiang, M. Dickinson, D.L. Pick, M. Sitti, and J. Yan, "Wing Transmission for a Micromechanical Flying Insect," IEEE International Conference on Robotics and Automation, 1509-1515 (2000).

${ }^{6}$ D. Lentink, N. Bradshaw, and S.R. Jongerius, "Novel Micro Aircraft Inspired by Insect Flight," Comparitive Biochemistry and Physiology A-Molecular and Integrative Physiology 146, No. 4, 133-134 (2007).

${ }^{7}$ T.N. Pornsin-Sirirak, M. Keenon, S.W. Lee, Y.C. Tai, J. Grasmeyer, H. Nassef, and C.M. Ho, "MEMS Wing Technology for a Battery-Powered Ornithopter," $13^{\text {th }}$ IEEE International Conference on Micro Electro Mechanical Systems, 799-804. (2000).

${ }^{8}$ C.P. Ellington, Limitations on Animal Flight Performance. Journal of Experimental Biology 160, 71-91. (1991).

${ }^{9}$ G.R. Spedding and P.B.S. Lissaman, Technical Aspects of Microscale Flight Systems. Journal of Avian Biology 29, No. 4, 458468. (1998).

${ }^{10}$ C.P. Ellington, Novel Aerodynamics of Insect Flight: Applications to Micro-Air Vehicles. Jornal of Experimental Biology 202, 3439-3448. (1999).

${ }^{11}$ U.M.L. Norberg, Structure, Form, and Function of Flight in Engineering and the Living World. Journal of Morphology 252, No. 1, 52-81. (2002).

${ }^{12}$ K.V. Rozhdestvensky and V.A. Ryzhov, Aerohydrodynamics of Flapping-Wing Propulsors. Progress in Aerospace Sciences 39, 585-633. (2003).

${ }^{13}$ S. Ho, H. Nassef, N. Pornsin-Sirirak, Y.C. Tai, and C.M. Ho, Unsteady Aerodynamics and Flow Control for Flapping Wing Flyers. Progress in Aerospace Sciences 39, No. 8, 635-681. (2003).

${ }^{14}$ D. Pines and F. Bohorquez, Challenges Facing Future Micro-Air-Vehicle Development. Journal of Aircraft 43, No. 2, $290-305$. (2006).

${ }^{15}$ Z. J. Wang, Dissecting Insect Flight, Annual Review of Fluid Mechanics 37, 183-210. (2005).

${ }^{16}$ S.A. Ansari, R. Zbikowski, and K. Knowles, Aerodynamic Modeling of Insect-like Flapping Flight for Micro Air Vehicles. Progress in Aerospace Sciences 42, 129-172. (2006).

${ }^{17}$ F. Togashi, Y. Ito, M. Murayama, K. Nakahashi, and T. Kato, Flow Simulation of Flapping Wings of an Insect Using Overset Unstructured Grid. AIAA Paper 2001-2619. (2001).

${ }^{18}$ H. Aono, W. Shyy, and H. Liu, Vortex Dynamics in Near Wake of a Hovering Hawkmoth. AIAA Paper 2008-583. (2008).

${ }^{19}$ Z.J. Wang, M. B. Birch, and M.H. Dickinson, Unsteady Forces and Flows in Low Reynolds Number Hovering Flight: TwoDimensional Computations vs Robotic Wing Experiments. Journal of Experimental Biology 207, 461-474. (2004).

${ }^{20}$ W. Shyy and H. Liu, Flapping Wings and Aerodynamic Lift: The Role of Leading-Edge Vortices. AIAA Journal 45, No. 12. (2007).

${ }^{21}$ W. Shyy, Y. Lian, J. Tang, H. Liu, P. Trizila, B. Stanford, P. Ifju, L. Bernal, C. Cesnik and P. Friedmann, Computational Aerodynamics of Low Reynolds Number Plunging, Pitching and Flexible Wings. Acta Mechanica Sinica 24, No. 4, 351373. (2008).

${ }^{22}$ Y. Liu and M. Sun, Wing kinematics measurement and aerodynamics of hovering droneflies. Journal of Experimental Biology 211, 2014-2025. (2008).

${ }^{23}$ J. Tang, D. Viieru, and W. Shyy, Effects of Reynolds Number and Flapping Kinematics on Hovering Aerodynamics. AIAA Journal 46, 967-976. (2008).

${ }^{24}$ D.F. Kurtulus, L. David, A. Farcy, and N. Alemdaroglu, Aerodynamic Characteristics of Flapping Motion in Hover. Experiments in Fluids 44, 23-36. (2008).

${ }^{25}$ F.M. Bos, D. Lentik, B.W. van Oudheusden, and H. Bijl, Influence of Wing Kinematics on Aerodynamic Performance in Hovering Insect Flight. Journal of Fluid Mechanics 594, 341-368. (2008).

${ }^{26}$ P. Trizila, C. Kang, M.R. Visbal, and W. Shyy, Unsteady Fluid Physics and Surrogate Modeling of Low Reynolds Number, Flapping Airfoils. AIAA Paper 2008-3821. (2008).

${ }^{27}$ S.P. Sane and M.H. Dickinson, The Control of Flight Force by a Flapping Wing: Lift and Drag Production. Journal of Experimental Biology 204, 2607-2626. (2001).

${ }^{28}$ R. Zbikowski, On aerodynamic modeling of an insect-like flapping wing in hover for micro air vehicles. Phil. Trans. R. Soc. Lond. A 360, 273-290. (2002).

${ }^{29}$ C.T. Hsieh, C.C. Chang, and C.C. Chu, Revisiting the aerodynamics of hovering flight using simple models. Journal of Fluid Mechanics 623, 121-148. (2009).

${ }^{30} \mathrm{~K}$. Taira and T. Colonious, Three-dimensional flows around low-aspect-ratio flat-plate wings at low Reynolds numbers. Journal of Fluid Mechanics 623, 187-207. (2009).

${ }^{31}$ C.P. Ellington, C. van den Berg, A.P. Willmott, and A.L.R. Thomas, Leading-Edge Vortices in Insect Flight. Nature 384, 626630. (1996).

${ }^{32} \mathrm{P}$. Freymuth, Thrust generation by an airfoil in hover modes. Experiments in Fluids 9, 17-24. (1990).

${ }^{33}$ S.N. Fry, R. Sayaman, and M.H. Dickinson, The Aerodynamics of Free-Flight Maneuvers in Drosophila. Science 300, $295-$ 298. (2003).

${ }^{34} \mathrm{M}$. Sun and J. Tang, Unsteady aerodynamic force generation by a model fruit fly wing in flapping motion. Journal of Experimental Biology 205, 55-70. (2002).

${ }^{35}$ W. Shyy, P. Trizila, C. Kang, and H. Aono, Can Tip Vortices Enhance the Lift of a Flapping Wing?, AIAA Journal 47, 289293. (2009).

${ }^{36}$ R. Kamakoti, S. Thakur, J. Wright, and W. Shyy, Validation of a new parallel all-speed CFD code in a rule-based framework for multidisciplinary applications. AIAA Paper 2006-3063. (2006). 
${ }^{37}$ W. Shyy, A Study of Finite Difference Approximations to Steady-State, Convection-Dominated Flow Problems. Journal of Computational Physics 57, No. 3, 415-438. (1985).

${ }^{38}$ W. Shyy, Computational Modeling for Fluid Flow and Interfacial Transport. (Elsevier, 1994).

${ }^{39}$ J. Blazek, Computational Fluid Dynamics: Principles and Applications. (Elsevier, 2001).

${ }^{40}$ E.A. Luke and T. George, Loci: A Rule-Based Framework for Parallel Multi-Disciplinary Simulation Synthesis. Journal of Functional Programming 15, No. 3, 477-502. (2005).

${ }^{41}$ M.R. Visbal and D.V. Gaitonde, High-Order-Accurate Methods for Complex Unsteady Subsonic Flows. AIAA Journal 37, No. 10, 1231-1239. (1999).

${ }^{42}$ M.R. Visbal and D.V. Gaitonde, On the Use of Higher-Order Finite-Difference schemes on Curvilinear and Deforming Meshes. Journal of Computational Physics 181, No. 1, 155-185. (2002).

${ }^{43}$ P. Trizila, C. Kang, M.R. Visbal, and W. Shyy, A Surrogate Model Approach in 2-D Versus 3-D Flapping Wing Aerodynamic Analysis, AIAA Paper 2008-5914. (2008).

${ }^{44}$ J.I. Madsen, W. Shyy, and R.T. Haftka, Response Surface Techniques for Diffuser Shape Optimization AIAA Journal 38, 15121518. (2000).

${ }^{45}$ W. Shyy, N. Papila, R. Vaidyanathan, and P.K. Tucker, Global Design Optimization for Aerodynamics and Rocket Propulsion Components. Progress in Aerospace Sciences 37, 59-118. (2001).

${ }^{46}$ N. Queipo, R.T. Haftka, W. Shyy, T. Goel, R. Vaidyanathan, and P.K. Tucker, Surrogate-Based Analysis and Optimization. Progress in Aerospace Sciences 41, 1-25. (2005).

${ }^{47}$ R.H. Myers and D.C. Montgomery, Response Surface Methodology: Process and Product Optimization Using Designed Experiments. (Wiley, 2002).

${ }^{48}$ J. Sacks, S.B. Schiller, and W.J. Welch, Design for Computer Experiments. Technometrics 31, No. 1, 41-47. (2008).

${ }^{49}$ B. Cheng and D.M. Titterington, Neural Networks: a Review from a Statistical Perspective. Statistical Science 9, 2-54. (1994).

${ }^{50}$ A.J. Smola and B. Scholkopf, A Tutorial on Support Vector Regression. Statistics and Computing 14, 199-222. (2004).

${ }^{51}$ T. Goel, R.T. Haftka, W. Shyy, and N.V. Queipo, Ensemble of Surrogates. Structural and Multidisciplinary Optimization 33, No. 3. (2007).

${ }^{52}$ F.A.C. Viana, R.T. Haftka, S.Valder, S. Butkewitsch, and M. Leal, Ensemble of Surrogates: a Framework based on Minimization of the Mean Integrated Square Error. AIAA Paper 2008-1885. (2008).

${ }^{53}$ J.C.R. Hunt, A.A. Wray, and P. Moin, Eddies, Streams, and Convergence Zones in Turbulent Flows. Report CTR-S88 Center for Turbulence Research. (1988).

${ }^{54} \mathrm{~T}$. Weis-Fogh, Energentics of Flight in Hummingbirds and in Drosophila. Journal of Experimental Biology 56, 79-104. (1972).

${ }^{55} \mathrm{~T}$. Weis-Fogh, Quick Estimates of Flight Fitness in Hovering Animals, Including Novel Mechanisms for Lift Production. Journal of Experimental Biology 59, 169-230. (1973).

${ }^{56}$ C.P. Ellington, The Aerodynamics of Hovering Insect Flight. III. Kinematics. Philosophical Transactions of the Royal Society of London, Series B 305, 41-78. (1984).

${ }^{57}$ J.D. Anderson, Fundamentals of Aerodynamics, $3^{\text {rd }}$ ed. (McGraw Hill, NewYork, 2001).

${ }^{58}$ K.M. Miettinen, Nonlinear Multiobjective Optimization ( Kluwer Academic Publishers, Boston, 1999). 